\title{
Intrinsic dissipation in nanomechanical resonators due to phonon tunneling
}

\author{
I. Wilson-Rae* \\ Departamento de Física Teórica de la Materia Condensada, \\ Universidad Autónoma de Madrid, 28049 Madrid, Spain and \\ Technische Universität München, D-85748 Garching, Germany.
}

(Dated: October 23, 2018)

\begin{abstract}
State of the art nanomechanical resonators present quality factors $Q \sim 10^{3}-10^{5}$, which are much lower than those that can be naively extrapolated from the behavior of micromechanical resonators. We analyze the dissipation mechanism that arises in nanomechanical beam-structures due to the tunneling of mesoscopic phonons between the beam and its supports (known as clamping losses). We derive the environmental force spectral density that determines the quantum Brownian motion of a given resonance. Our treatment is valid for low frequencies and provides the leading contribution in the aspect ratio. This yields fundamental limits for the $Q$-values which are described by simple scaling laws and are relevant for state of the art experimental structures. In this context, for resonant frequencies in the $0.1-1 \mathrm{GHz}$ range, while this dissipation mechanism can limit flexural resonators it is found to be negligible for torsional ones. In the case of structureless $3 \mathrm{D}$ supports the corresponding environmental spectral densities are Ohmic for flexural resonators and super-Ohmic for torsional ones, while for $2 \mathrm{D}$ slab supports they yield $1 / f$ noise. Furthermore analogous results are established for the case of suspended semiconducting single-walled carbon nanotubes. Finally, we provide a general expression for the spectral density that allows to extend our treatment to other geometries and illustrate its use by applying it to a microtoroid. Our analysis is relevant for applications in high precision measurements and for the prospects of probing quantum effects in a macroscopic mechanical degree of freedom.
\end{abstract}

PACS numbers: 85.85.+j,03.65.Yz,63.22.+m,07.10.Cm

\section{INTRODUCTION}

Nanomechanical resonators offer a host of novel applications $\$^{1213}$ in high precision measurements $\frac{\sqrt{415}}{}$ and may provide a new arena for probing fundamental aspects of quantum physics ${ }^{6}$. A prominent example of such novel applications is scaling magnetic resonance force microscopy ${ }^{7}$ (MRFM) down to the level of single-spin detection. This degree of sensitivity would allow, for example, three-dimensional imaging of individual biomolecules with atomic-scale resolution. In these microscopes a magnetic particle ${ }^{8}$ mounted on a cantilever interacts with the nuclear or electron spins in the sample via the magnetic dipole force. Hence as in many other applications the relevant mechanical resonator has the beam geometry on which we will mainly focus in this paper ${ }^{9}$. Beyond microscopy, an interesting issue is whether in the nanoscale regime such a device could be used to probe the quantum state of a single spin 10 .

A single spin $1 / 2$ coupled to a harmonic oscillator, namely the Jaynes-Cummings model, constitutes a fundamental system in quantum optics ${ }^{11}$. Physical realizations of this system have proved invaluable in probing and understanding quantum phenomena ${ }^{12 \mid 13}$. These investigations have ranged from studying the quantum-classical interface to proof-of-principle demonstrations of the basic building blocks of quantum information processing. These developments were enabled by the advent of high$Q$ resonators for optical and microwave photons and of conservative harmonic traps for atoms. In these systems the relevant harmonic oscillator is furnished, respectively, by a single normal mode of the electromagnetic field or

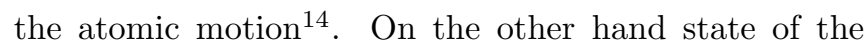
art semiconductor nanostructures can support mechanical resonances with quality factors $Q \sim 10^{3}-10^{5}$ and frequencies approaching the $\mathrm{GHz}$ regime ${ }^{3}$. For some of these structures measurements of mechanical displacements with a sensitivity approaching the quantum limit have been achieved by exploiting capacitive coupling to a single electron transistor $\frac{15 / 16}{}$. These developments suggest the possibility of realizing a quantum phononics realm in complete analogy to quantum optics 6117 . In this case the relevant harmonic oscillator would be furnished instead by a mechanical resonance and the role of the pseudospin could be played by a capacitively coupled Cooper pair box ${ }^{[18 \mid 19}$ or an excitonic transition of an embedded self-assembled quantum dot $\frac{20}{20}$. Along these lines one could envisage observing quantum jumps due to the discrete nature of phonons and realizing quantum state engineering of non-classical states of motion 21 . In addition to semiconductor planar heterostructure realizations there are other promising possibilities like suspended single walled carbon nanotubes 22123124 (SWNT), nanowires ${ }^{25}$, and single crystal diamond beams with embedded nitrogen vacancy color centers 26 . Another alternative for furnishing the non-linearity needed to induce non-classical behavior in mesoscopic mechanical oscillators are optomechanical schemes in which the resonator couples via radiation pressure to an optical cavity $27 / 28$. This venue has recently witnessed significant experimental progress 29 towards achieving ground state cooling which is highly desirable to enable quantum effects30. Finally, for resonators with sufficiently small effective masses and high bending rigidity, yet another alternative 
that has been considered is to use the Euler instability 31 .

The realization of all of the aforementioned applications of nanomechanical systems hinges on understanding and controlling the intrinsic dissipation and noise mechanisms 32 that limit their coherent dynamics. For centimeter-scale semiconductor micromechanical systems $Q \sim 10^{8}$ have been measured at low temperatures ${ }^{2}$. On the other hand when these devices are shrunk to the nanometer-scale these values decrease dramatically to $Q \sim 10^{3}-10^{5}$. Early work suggested that the increase in surface-to-volume ratio combined with surface effects might be invoked as a plausible explanation of this phenomenon ${ }^{2}$. Subsequently it was realized that elastic wave radiation into the supports - the so-called clamping losses ${ }^{3}$ — could play an important role leading to non-trivial scaling laws for the $Q$-values with the aspect ratio that are intimately related to the low frequency behavior of the corresponding transmission coefficients (cf. Sec. IV and Refs. 33343536]).

If we consider the environment of the mechanical resonator responsible for its dissipation, the $Q$-value is determined by the pole of the ensuing modified propagator for the resonator's normal coordinate that corresponds to its resonant frequency $\omega_{R}$. If one adopts a CaldeiraLeggett mode $\sqrt[37 / 38]{3}$ - i.e. the environment is assumed to consist of a thermal ensemble of harmonic oscillators - with a linear coupling to the environment, this propagator can be obtained exactly from the environmental force spectral density $I(\omega)$ and the $Q$-value is found to be temperature independent. Thus, the quantum Brownian motion of the normal coordinate $\hat{X}_{R}$ associated with a given resonance is characterized by the following generalized equation of motion

$$
\ddot{\hat{X}}_{R}(t)+\int_{0}^{t} \mathrm{~d} t^{\prime} \gamma\left(t-t^{\prime}\right) \dot{\hat{X}}_{R}\left(t^{\prime}\right)+\omega_{R}^{2} \hat{X}_{R}(t)=\hat{\xi}(t)
$$

and is completely determined by the function $I(\omega)=$ $\omega \int_{-\infty}^{\infty} \mathrm{d} t \gamma(t) e^{i \omega t} / 2 \omega_{R}$ where $\gamma(t)$ is the symmetric dissipation-kerne ${ }^{39}$ [cf. Eq. 21] and Sec. II and $\hat{\xi}(t)$ corresponds to the environmental noise. When the standard Markov approximation is warranted $\gamma(t) \sim \delta(t)$ and the $Q$-value is determined by

$$
\frac{1}{Q}=\frac{I\left(\omega_{R}\right)}{\omega_{R}} .
$$

Within this approximation the value $I\left(\omega_{R}\right)$ is the only relevant information about the spectral density, Eq. (1) can be interpreted as a quantum Langevin equation, and we have the standard relation

$$
\frac{1}{Q}=\frac{\langle\dot{E}\rangle}{\omega_{R}\langle E\rangle}
$$

where $E$ is the total energy stored in the resonator's degree of freedom. This is normally valid for sufficiently high $Q$ and low temperature if $I(\omega)$ is smooth enough, or for high temperatures if $I(\omega)$ scales linearly with
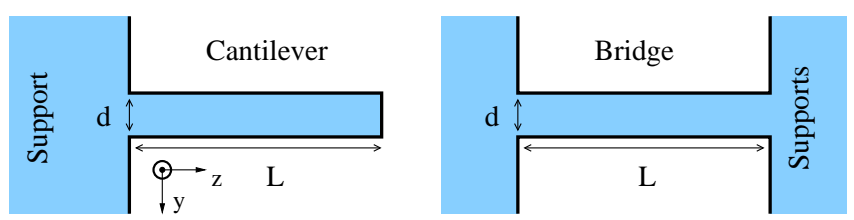

FIG. 1: Schematic diagram of suspended mechanical beam structures presenting abrupt junctions with the supports. Throughout the paper the $z$-axis corresponds to the beam's axis, while the $x$ and $y$ axes are the principal axes of inertia of the beam's cross section at the left junction (the origin is set at the center of mass of the latter).

frequency 38 (classical Langevin). However in many instances it is desirable to go beyond the Markov approximation and more precise knowledge about $I(\omega)$ is needed. Examples of these are the cases discussed above in which the resonator interacts with a single spin $1 / 2$ system if the coupling is relatively strong and cases in which the $Q$ is not high enough or the temperature low enough. Currently there exists no microscopic derivation of Eq. (1) and of the underlying Caldeira-Leggett Hamiltonian and corresponding spectral density $I(\omega)$, for which customarily an ohmic dependence is assumed.

For nanometer-sized suspended monocrystalline beam structures at low temperatures, the relevant phonon mean free path can become larger than the beam's length. In the case of an insulating system, a prominent consequence of the ballistic regime that results is the quantization of thermal conductance 41 in units of $G_{t h}=\pi^{2} k_{R}^{2} T / 3 h$, which has been demonstrated experimentally 2 . In this low temperature, low frequency regime and for small deflections, anharmonicity becomes irrelevant for the analysis of dissipative effects $9 \mid 43 / 44$. Thus, in this paper we analyze the ideal limit, that ensues for the low-lying resonances of an insulating beam close to equilibrium at low enough temperatures, in which phonon tunneling between the beam and its supports is the only source of thermal noise and dissipation. To this effect the vibrations of the whole structure are described by a purely harmonic Hamiltonian. Its normal modes, discussed in the following section, form a continuum. As will become clear below, this feature is inherited from the supports that thus provide thermal phonon reservoirs. When the systems under consideration deviate from the aforementioned ideal scenario (e.g. the vacuum is imperfect, the beam's material is amorphous or surface effects are relevant) there will be other contributions to the mechanical damping that will add incoherently ${ }^{3}$. However it should be stressed that in all such cases our treatment is valid for the contribution to the dissipation arising from the vibrational degrees of freedom and thus our results will provide an upper bound for the $Q$-values.

Beam nanostructures for which high $Q$-values have been measured normally involve abrupt junctions with the supports. This can be intuitively understood in terms of impedance mismatch for the propagation of elastic 
waves. We will focus mainly on this type of structures, as depicted in Fig. 1, and consider phonon frequencies that correspond to $k \sim 2 \pi / L \ll 2 \pi / d$, where $k$ is the wavevector along $z$ inside the beam, $L$ the beam's length, and $d$ the typical dimension of the cross section (cf. Fig. 1 and App. B. It is clear that for this beam geometry the reciprocal of the aspect ratio $d / L$ provides a natural small parameter that will underpin our analysis of the non-trivial matching at the abrupt junctions. Given that we are interested in phonons with wavelengths much longer than the lattice constant, the continuum limit constitutes a good approximation. This yields for the supports 3D isotropic elasticity ${ }^{9 / 45 / 46}$, if one further neglects the possible anisotropy - or 2D "thin plate elasticity" for slab supports of thickness much smaller than the phonon wavelength 33 . If we consider the beam there are basically two scenarios: $(i)$ all the characteristic dimensions of the cross section are much larger than the lattice constant (e.g. semiconductor planar heterostructures) or (ii) there are characteristic dimensions comparable to the lattice constant (e.g. semiconducting SWNTs and small-radius nanowires). In case $(i)$ one can start from 3D isotropic elasticity and obtain the effective theory valid for phonon wavelengths much larger than $d$ known as "thin rod elasticity" is completely determined by the linear mass density $\mu_{b}$, the mean axial moment of inertia $\left\langle r^{2}\right\rangle_{S}$, and the extensional, torsional and bending rigidities; and leads to the following dispersion relations for the four low frequency branches

$$
\omega_{\beta}(k)=\tilde{c}_{\beta} k^{p_{\beta}} \quad p_{c}=p_{t}=1, p_{v}=p_{h}=2 .
$$

Here $\beta$ is the branch index: $c$ for compression, $t$ for torsion, $v$ for vertical bending and $h$ for horizontal bending [the prefactors $\tilde{c}_{\beta}$ are given in App. B. Eq. (B9)]. Though for propagation inside the beam this effective theory will suffice, resort to the underlying 3D "microscopic" theory will allow for a rigorous treatment of the matching at the junction (cf. Sec. IV) - an analogous procedure is feasible for a thin plate geometry ${ }^{33}$ with the "microscopic" theory furnished by $2 \mathrm{D}$ thin plate elasticity. In case $(i i)$ it is well understood that the effective long wavelength theory is formally equivalent to TRE but the standard "bulk" relations between the rigidities are no longer warranted 4 . Whence heuristic considerations allow to extend our treatment of the non-trivial matching at the abrupt junctions to this case with suitably redefined $\tilde{c}_{\beta}$ - when considering this extension we will focus mainly on SWNTs. Thus, our treatment will amount to a derivation of the leading contribution in the aspect ratio to the environmental spectral densities $I(\omega)$ associated with phonon tunneling induced noise starting from the underlying microscopic lattice Hamiltonian.

The relevance of the ensuing results is twofold: $(i)$ they furnish a very general understanding of clamping losses determining the corresponding $Q$-values for a wide range of experimentally relevant structures, and (ii) they provide an instance for which a microscopic derivation of the quantum dissipative dynamics of a "macroscopic" mechanical degree of freedom can be given, which reduces exactly to a Caldeira-Leggett model $\frac{40 \mid 48 / 49}{\text {. The latter }}$ is essentially a derivation of the basic Hamiltonian for a lossy 1D phonon-cavity and brings together aspects of the analogous problem in quantum optics 50 and the ballistic transport in a mesoscopic wire ${ }^{41}$. In this respect it should be noted that the Markov approximation, which is customarily used for the Brownian motion of mechanical resonators, is not assumed in our derivation but instead its range of validity emerges from the behavior of the spectral densities $I(\omega)$ that we calculate and that allow (if necessary) to go beyond the Markovian regime - for these aspects the reader is referred to the vast literature on quantum dissipation 38 . Thus, the validity (for large aspect ratios) of Eq. (2), that will be used to determine the $Q$-values, is established rather than postulated.

We will first present our main results (cf. Subsec. IA) and subsequently give their derivation in the following Sections. For the sake of clarity we will mainly focus on a specific model with maximally symmetric 3D structureless supports and the beam having the aforementioned property $(i)$ [cf. Subsec. II A], and outline how the treatment can be extended (cf. App. C) by referral to this concrete realization. We will discuss both the bridge and cantilever geometries (cf. Fig. 1). Finally, we will also outline how this approach can be generalized to other geometries and illustrate this further extension for the experimentally relevant case of a microtoroid 28151 .

\section{A. Environmental force spectral densities and $Q$-values for each resonance}

To study the dissipation induced by the coupling to the supports it proves useful to introduce the concept of an "effective environmental density of states" for each branch given by $\tilde{\rho}_{\beta}(\omega)$. These functions will be defined in Sec. IV. There we will find that for 3D supports they bear simple relations with properties of the decoupled support - i.e. subject to free boundary conditions that are closely related to its density of states (DOS); namely, the displacement and angle (twist) vacuum spectrum at the junction ${ }^{62}$. The cornerstone of our analysis will be furnished by the following novel pair of fundamental relations, each of which completely specifies the environmental force spectral density for a given resonance to lowest order in the reciprocal of the aspect ratio $d / L$ :

$$
\begin{aligned}
& I_{n, \beta}(\omega)=\delta C_{n, \beta}\left[\frac{\mathrm{d} \omega_{\beta}}{\mathrm{d} k}\left(\omega_{n, \beta}\right)\right]^{2} \frac{\tilde{\rho}_{\beta}(\omega)}{2 L} \frac{\omega_{n, \beta}}{\omega} \\
& I_{n, \beta}(\omega)=\delta C_{n, \beta} \frac{\mathrm{d} \omega_{\beta}}{\mathrm{d} k}(\omega) \frac{\tau_{\beta}(\omega)}{2 L}\left(\frac{\omega_{n, \beta}}{\omega}\right)^{p_{\beta}} .
\end{aligned}
$$




\begin{tabular}{|c|c|c|c|c|c|c|}
\hline & \multicolumn{2}{|c|}{ General Relations (3D) } & \multicolumn{2}{|c|}{ Monolithic structure $\left(\sigma=\frac{1}{3}\right)$} & \multicolumn{2}{|l|}{ Semiconducting SWNT $\left(\sigma_{s}=\frac{1}{3}\right)$} \\
\hline & $I_{n}(\omega)$ & $Q_{n}$ & $Q_{n}(L, \mathrm{w}, \mathrm{t})$ & $\begin{array}{l}\text { Typical } \\
\text { value for } \\
150 \mathrm{MHz}\end{array}$ & $Q_{n}(L, R)$ & $\begin{array}{c}\text { Typical } \\
\text { value for } \\
1 \mathrm{GHz} \\
\end{array}$ \\
\hline Compression & $\frac{\omega}{Q_{n}}$ & $\frac{\rho_{s} c_{t}^{3} L}{2 \delta \mu_{b} \tilde{c}_{c}^{3} \tilde{u}_{c}(\alpha) k_{n}}$ & $\frac{0.88}{\pi \delta} \frac{L^{2}}{\mathrm{wt}} \frac{1}{n+\frac{\delta}{2}}$ & $3.2 \times 10^{4}$ & $\frac{0.14}{\pi \delta} \sqrt{\frac{\sigma_{G}}{h \rho_{s}}\left(\frac{E_{s}}{E_{b}}\right)^{3}} \frac{L^{2}}{h R} \frac{1}{n+\frac{\delta}{2}}$ & $1.5 \times 10^{5}$ \\
\hline Torsion & $\frac{\omega^{3}}{Q_{n} \omega_{n}^{2}}$ & $\frac{\rho_{s} c_{t}^{5} L}{2 \delta \mu_{b}\left\langle r^{2}\right\rangle \tilde{c}_{t}^{5} \tilde{u}_{t}\left(\alpha, \gamma_{z}\right) k_{n}^{3}}$ & $\frac{4.1}{\pi^{3} \delta} \frac{\mathrm{w}^{2} L^{4}}{\mathrm{t}^{6}} \frac{1}{\left(n+\frac{\delta}{2}\right)^{3}}$ & $7.6 \times 10^{9}$ & $\frac{2.3}{\pi^{3} \delta} \sqrt{\left(\frac{\sigma_{G}}{h \rho_{s}}\right)^{3}\left(\frac{E_{s}}{E_{b}}\right)^{5}} \frac{L^{4}}{h R^{3}} \frac{1}{\left(n+\frac{\delta}{2}\right)^{3}}$ & $1.3 \times 10^{11}$ \\
\hline $\begin{array}{l}\text { Vertical } \\
\text { bending }\end{array}$ & $\frac{\omega}{Q_{n}}$ & $\frac{\rho_{s} c_{t}^{3} L}{4 \delta C_{n} \mu_{b} \tilde{c}_{v}^{3} \tilde{u}_{v}(\alpha) k_{n}^{4}}$ & $\frac{3.9}{\pi^{4} \delta C_{n}} \frac{L^{5}}{\mathrm{wt}^{4}}\left(\frac{3 \pi}{2 k_{n} L}\right)^{4}$ & $9.6 \times 10^{5}$ & $\frac{0.043}{\pi^{4} \delta C_{n}} \sqrt{\frac{\sigma_{G}}{h \rho_{s}}\left(\frac{E_{s}}{E_{b}}\right)^{3}} \frac{L^{5}}{h R^{4}}\left(\frac{3 \pi}{2 k_{n} L}\right)^{4}$ & $4.2 \times 10^{6}$ \\
\hline $\begin{array}{l}\text { Horizontal } \\
\text { bending }\end{array}$ & $\frac{\omega}{Q_{n}}$ & $\frac{\rho_{s} c_{t}^{3} L}{4 \delta C_{n} \mu_{b} \tilde{c}_{h}^{3} \tilde{u}_{h}(\alpha) k_{n}^{4}}$ & $\frac{3.9}{\pi^{4} \delta C_{n}} \frac{L^{5}}{\mathrm{tw}^{4}}\left(\frac{3 \pi}{2 k_{n} L}\right)^{4}$ & $3.9 \times 10^{5}$ & $\frac{0.043}{\pi^{4} \delta C_{n}} \sqrt{\frac{\sigma_{G}}{h \rho_{s}}\left(\frac{E_{s}}{E_{b}}\right)^{3}} \frac{L^{5}}{h R^{4}}\left(\frac{3 \pi}{2 k_{n} L}\right)^{4}$ & $4.2 \times 10^{6}$ \\
\hline
\end{tabular}

TABLE I: First and second columns: general formulas for the environmental force spectral densities $I_{n}(\omega)$ and the $Q$-values $Q_{n}$ corresponding to the different resonances of a beam suspended from structureless $3 \mathrm{D}$ support(s) $-n=0,1, \ldots$ labels the harmonics for each of the four branches. These general formulas are specialized for two cases: (third column) a monolithic structure with rectangular beam cross section of thickness $\mathrm{t}$ and width $\mathrm{w}$, and (fifth column) a suspended semiconducting SWNT of radius $R$. The nanotube is modeled as a cylindrical shell of effective thickness $h$ and Poisson ratic $\sigma_{b}=0.19$. All formulas are valid for both the cantilever $(\delta=1)$ and bridge $(\delta=2)$ geometries. The dispersion-relation prefactors $\tilde{c}_{\beta}$ are given in Eq. (B9), $\mu_{b}$ is the linear mass density of the beam (for the nanotube $\mu_{b}=2 \pi R \sigma_{G}$, where $\sigma_{G}$ is the surface density of graphene), $\rho_{s}$ and $c_{t}$ are, respectively, the mass density and transverse speed of sound for the supports' material, and $\left\langle r^{2}\right\rangle \equiv I_{z} / S$ is the beam's mean axial moment of inertia. In the case of torsion (second row), for the monolithic structure (third and fourth columns), we specialize for $\mathrm{t} \ll \mathrm{w}$ so that $\gamma_{z}^{2} \approx 1$ and the torsional rigidity $\underline{45}^{4}$ reads $C \approx E_{b} \mathrm{wt}^{3} / 6\left(1+\sigma_{b}\right)$ [for a cylindrical shell we have instead $\left.C=E_{b} \pi h R^{3} /\left(1+\sigma_{b}\right)\right]-E_{b}\left[E_{s}\right]$ is the Young's modulus for the material of the beam [support(s)]. The dimensionless displacements and angles $\tilde{u}_{\beta}$, given in Subsec. IV C (cf. Fig. 3), take the following values for $\sigma_{s}=1 / 3\left[\alpha \equiv\left(1-2 \sigma_{s}\right) / 2\left(1-\sigma_{s}\right)=1 / 4\right]: \tilde{u}_{c}(1 / 4)=0.13, \tilde{u}_{t}\left(1 / 4, \gamma_{z}\right)=1 / 12 \pi+0.019 \gamma_{z}^{2}$, and $\tilde{u}_{v / h}(1 / 4)=0.12$, where $\gamma_{z} \equiv\left(I_{y}-I_{x}\right) / I_{z}$ and we have defined $\tilde{u}_{t}\left(\alpha, \gamma_{z}\right)=\tilde{u}_{t}^{(A)}+\tilde{u}_{t}^{(S)}(\alpha) \gamma_{z}^{2}$. The resulting typical values for the $Q_{n}$ (fourth and sixth columns) correspond to the lowest lying resonances of bridge geometries (wavevectors ${ }^{a} k_{0, c / t}=\pi / L$ and $k_{0, v / h} \approx 1.51 \pi / L$ ) with a different length for each branch $\left(L_{\beta}\right)$ chosen so that the comparison is made for equal frequencies ${ }^{b}: 150 \mathrm{MHz}$ for the monolithic structure (parameters: $\mathrm{w}=100 \mathrm{~nm}$ and $\mathrm{t}=20 \mathrm{~nm}$ ) and $1 \mathrm{GHz}$ for the nanotube [parameters: $h=0.66 \AA(\mathrm{cf}$. Ref. 52 ), $\sigma_{G}=7.7 \times 10^{-7} \mathrm{Kg} / \mathrm{m}^{2}, E_{b}=1 \mathrm{TPa}$ (cf. Ref. [22), and $R=1 \mathrm{~nm}$; we assume Si supports: $\rho_{s}=2.3 \times 10^{3} \mathrm{Kg} / \mathrm{m}^{3}, E_{s}=112 \mathrm{GPa}$.

${ }^{a}$ For the cantilever geometry these would be instead $k_{0, c / t}=\pi / 2 L$ and $k_{0, v / h} \approx 0.60 \pi / L$.

${ }^{b}$ For the monolithic case (nanotube): $L_{c}=23 \mu(2.3 \mu), L_{t}=4.7 \mu(1.5 \mu), L_{v}=0.98 \mu(0.11 \mu), L_{h}=2.4 \mu(0.11 \mu)$.

Here $n=0,1, \ldots$ labels the harmonics for each branch $\beta$ (i.e. $\left.\omega_{R} \rightarrow \omega_{n, \beta}\right), \delta=1,2$ is the number of supports,

$$
C_{n, \beta}= \begin{cases}1 & \text { for } \beta=c, t \\ \left(\tanh ^{2} \frac{k_{n, v / h} L}{2}\right)^{(-1)^{n}} & \text { for } \beta=v, h\end{cases}
$$

and the bare resonant wavevectors $k_{n, \beta} \equiv \omega_{\beta}^{-1}\left(\omega_{n, \beta}\right)$ are determined by the TRE solutions for clamped-clamped (clamped-free) boundary conditions in the case of the bridge (cantilever) geometry $\sqrt{63}$. The first relation expresses the spectral density in terms of $\tilde{\rho}_{\beta}(\omega)$. The second relation involves the transmission coefficient $\tau_{\beta}(\omega)$ from a semi-infinite beam into the support at a single junction which can be interpreted as the probability for an incident phonon with frequency $\omega$ to tunnel into the support. As will be discussed further in the next Section and derived rigorously in Subsec. IVA, for phonon frequencies $\omega \rightarrow 0$ the beam and the supports become decoupled so that the junction plays a role analogous to a tunnel barrier. Whence relation (6) allows to interpret the losses to the supports in terms of phonon tunneling. More precisely, it provides a rigorous footing for the heuristic formula to describe this dissipation mechanism set forth by Cross and Lifshitz in Ref. 33. based upon Eq. (3), namely,

$$
\frac{1}{Q} \sim \frac{\delta}{2 L} \frac{\mathrm{d} \omega_{\beta}}{\mathrm{d} k}\left(k_{n}\right) \frac{\tau_{\beta}\left(k_{n}\right)}{\omega_{\beta}\left(k_{n}\right)}=\frac{\delta p_{\beta}}{2} \frac{\tau_{\beta}\left(k_{n}\right)}{k_{n} L},
$$

where the implicit dimensionless prefactor is expected to be of order unity and have the latter as its limit for $n \rightarrow \infty$ - henceforth we drop the branch index of the $k_{n}, \omega_{n}$.

The above approximation emerges from considering a phonon wavepacket that bounces back and forth between both ends of the beam. Naturally, it should be adequate for large $n$ and provides a simple intuitive description of this dissipation mechanism in terms of phonon tunneling at the junctions. Here we have added a factor of $\delta / 2$, 
where $\delta$ is the number of supports, to account for both the bridge and cantilever geometries. Equation (8) can be obtained from Eqs. (2) and (6) which allow in addition to determine that the corresponding dimensionless prefactor is given by $C_{n, \beta}$ - i.e. for the non-dispersive branches it is exactly unity for all $n$ and only deviates from unity for the low-lying bending resonances.

The general relation between the transmission coefficient at a single junction and the effective environmental DOS concomitant to the pair of relations (5), (6) will be proved in Sec. IV where expressions for the functions $\tau_{\beta}(\omega)$ and $\tilde{\rho}_{\beta}(\omega)$ are derived explicitly for 3D supports [cf. Eqs. (58)-(63)]. The latter together with Eqs. (5) and (2) allow us to obtain formulas for the $Q$-values of all the low-frequency resonances that are given in the second column of Table [. The first column gives the resulting expression for the environmental force spectral density which turns out to be ohmic in all cases except for the torsional resonances. The third and fifth columns give examples of particular experimental relevance. Namely, a monolithic structure with rectangular cross section for the beam of width $w$ and thickness $t$ and a semiconducting suspended SWNT of radius $R$ - for the nanotube we use constants $\tilde{c}_{\beta}$ that correspond to the "continuum" shell approximation for the rigidities $52 \mid 53$. The validity of these results, which are adequate for low frequencies, will be borne out in full detail in the following Sections as we derive the Eqs. (5) and (6). While the latter will hold in all instances where any characteristic dimension of the supports is either much larger than $L$ or at most of order $d_{s} \lesssim d$, Table $\mathrm{I}$ focuses on the case in which the limit $d_{s} \rightarrow 0$ yields a $3 \mathrm{D}$ support with no characteristic dimension (e.g. an elastic half-space). Within this context the second column is general with an appropriate definition of the dimensionless displacements and angles $\tilde{u}$ that only depend on the supports' material Poisson ratio (cf. Sec. IV] and App. C). On the other hand the specific $\tilde{u}$ used in the examples — which will be calculated in Sec. IV - correspond to the maximally symmetric case of the half-space.

In the context of the applications already discussed, one focuses on a specific resonance and it can be argued that a sound figure of merit is afforded by the quantity $k_{B} T / \hbar \omega_{R} Q$. Therefore we compare the different types of resonances (for different lengths $L$ ) for the same resonant frequency $\omega_{R}$ (fourth and sixth columns). It is clear that for specified materials these results for the $Q$ values only depend on the ratios between the beam's dimensions. Thus, for semiconductor heterostructure realizations the corresponding formulas (second and third columns) hold all the way from the nanoscale to the macroscopic regime and are also applicable to micromechanical resonators. However, while for fabricated flexural resonators with sub-micron transverse dimensions aspect ratios $L / d \sim 10$ are not uncommor ${ }^{9}$, for typical micromechanical resonators used in MEMS $L / d \gtrsim 100$ rendering the above results an upper bound that would be hard to reach - note for comparison that the last two entries of the fourth column correspond, respectively, to $L / \mathrm{t}=49$ and $L / \mathrm{w}=24$. Finally, we note that for given 3D supports the above results are "universal" in the specific sense that their only dependence on properties of the beam is through $\mu_{b},\left\langle r^{2}\right\rangle_{S}$, and the prefactor of the TRE dispersion relations (4), quantities that specify its low-frequency effective theory. In particular, the scaling laws with the beam's length are completely general. Furthermore, for a monolithic structure the dependence on the material's Poisson ratio in the relevant range $1 / 5 \lesssim \sigma \lesssim 1 / 3$ is so weak (cf. Sec. IV) that the $Q$-value of a given resonance can be effectively regarded as a geometric property.

The other instance of interest to which Eqs. (5), (6) apply and that also yields simple scalings, is the case of slab 2D supports of thickness $d_{s}=\mathrm{t}$ equal to the thickness of the beam. The results we will obtain [cf. Sec. IV] Eq. [64)] for the frequency dependence of the $\tilde{\rho}_{\beta}(\omega)$ together with Eqs. (5), 22 imply the following

$$
I_{n, \beta \neq v}=\frac{\omega_{n}}{Q_{n}} \quad I_{n, v}=\frac{\omega_{n}^{2}}{Q_{n} \omega},
$$

which correspond to $1 / f$ noise 5455 . We note that the finite size of the slab provides a natural infrared cutoff. The corresponding $Q$-values (which with the exception of horizontal bending scale as the aspect ratio $L /$ w) have already been derived in Ref. 33] up to the prefactor $C_{n}$ discussed above.

Standard fabrication procedures ${ }^{9}$ normally result in an undercut of the support of size $d_{U}$ at least comparable to the width of the beam. Our analysis of the abrupt junction(s) with the support(s) given in Sec. IV and App. C implies that it is quantitatively correct to use the $3 \mathrm{D}$ model for the support(s) when $d_{U} \lesssim d$, and the 2D model discussed above when $d_{U} \gg L$. In addition, based on heuristic considerations, the $3 \mathrm{D}$ results for the $Q$-values

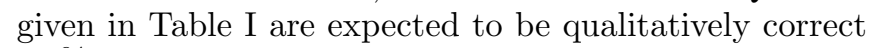
for ${ }^{64} d_{U}<L$.

Finally, it should be noted that a substantial part of our derivation of the spectral density for an isolated resonance ${ }^{65}$ is independent of the geometry. More precisely Eqs. (5) and (6) can be viewed as a specific instance of the following more general relation

$$
\begin{aligned}
I(\omega) \approx \frac{\pi}{2 \rho_{s}^{2} \omega_{R} \omega} \int_{q} \mid & \left.\int_{S} \mathrm{~d} r^{2}\left(\bar{u}_{R}^{\prime} \cdot \boldsymbol{\sigma}_{q}-\bar{u}_{q} \cdot \boldsymbol{\sigma}_{R}^{\prime}\right) \cdot \hat{n}\right|^{2} \\
& \times \delta[\omega-\omega(q)]
\end{aligned}
$$

where $\bar{u}_{q}(\bar{r})$ and $\boldsymbol{\sigma}_{q}(\bar{r})$ are the displacement and stress fields associated with scattering eigenmodes for the whole structure, labeled by $q$ [eigenfrequencies $\omega(q)]$, and $\bar{u}_{R}^{\prime}(\bar{r})$, $\boldsymbol{\sigma}_{R}^{\prime}(\bar{r})$ are the analogous fields for the resonator mode (cf. Section II). Here $S$ is the contact surface between the resonator and its support(s) and $\rho_{s}$ is the mass density of the latter. As will become clear in Subsec. VB the small parameter associated with the above approximation is $\left|\Delta_{I}\left(\omega_{R}\right)\right| / 2 \omega_{R}$, where $\Delta_{I}\left(\omega_{R}\right) / 2$ is the support-induced shift of the resonant frequency (cf. App. F). 


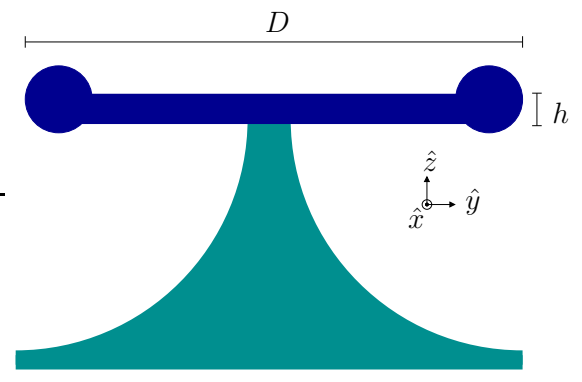

FIG. 2: Schematic diagram of a microtoroid structure with largest diameter $D$ and membrane thickness $h$ (the origin is set at the center of the contact area $S$ with the pedestal).

In all natural scenarios $\left|\Delta_{I}\left(\omega_{R}\right)\right| / 2 \omega_{R} \ll 1$ arises due to the abrupt nature of the junction(s) with the support(s) and the condition $\sqrt{S} k_{R} \ll 1-$ where $k_{R}$ is the typical wavevector associated with the resonator mode. The behavior as $S \rightarrow 0$ leads to two possibilities: (i) the limit is singular, or (ii) it defines a well-behaved resonator geometry that can be described by $3 \mathrm{D}$ elasticity. The beam geometry falls into case (i) for which $\bar{u}_{R}^{\prime}(\bar{r})$ is specified by clamped boundary conditions at $S$ (cf. Subsec. II B). In turn in case (ii) $\bar{u}_{R}^{\prime}(\bar{r})$ should satisfy free boundary conditions at the contact surface. An experimentally relevant example of the latter is afforded by microtoroids 28151 (cf. Figure 2). For such structures an heuristic treatment of the pedestal as a beam with adiabatically varying cross section allows us to obtain from Eq. 10 the following

$$
I(\omega) \approx \frac{\sqrt{\rho_{s} E_{s}}}{m_{R} \omega_{R}} S \tilde{u}_{R, z}^{2}(0) \omega
$$

for any axially symmetric isolated resonance. Here the resonator mode is normalized so that the normal coordinate corresponds to the elongation of the toroid's external radius, and $m_{R}$ and $\overline{\tilde{u}}_{R}(\bar{r})$ are, respectively, the corresponding effective mass and mode profile (cf. App. D). Equation (2) then yields for the corresponding $Q$-value

$$
Q \approx \frac{m_{R} \omega_{R}}{\sqrt{\rho_{s} E_{s}} S \tilde{u}_{R, z}^{2}(0)} .
$$

Typical values for the radial breathing mode of state of the art structures $\operatorname{arc} 51$ : $\omega_{R}=2 \pi \times 50 \mathrm{MHz}, m_{R}=$ $10^{-11} \mathrm{Kg}, S=\pi / 4 \times(0.5 \mu)^{2}$, and $\tilde{u}_{R, z}(0)=1 / 2$, which (for an Si substrate) result in $Q \approx 4 \times 10^{3}$. In addition to $\sqrt{S} k_{R} \ll 1$, the above approximation assumes that there is perfect impedance-match between the pedestal and the substrate, and that $\sqrt{S} \lesssim h$ - here $h$ is the smallest characteristic dimension of the resonator. It can be argued that for pedestals with lengths at least comparable to $2 \pi / k_{R}$ deviations from this adiabatic scenario will only increase the $Q$-value provided that $\omega_{R}$ does not coincide with a resonance of the pedestal. The derivation of Eqs. (10) and (11) is given in Appendix D.

\section{BASIC MODEL AND OUTLINE}

\section{A. Normal modes of the supports, the beam, and the whole structure}

We will make two simplifying assumptions: (i) the supports have typical dimensions that are much larger than the beam's length $L$ and for a bridge both of them are identical, and (ii) both the supports and the beam present reflection symmetries with respect to the $x z$ and $y z$ planes (cf. Fig. 1). The first assumption is adequate to describe a wide range of realistic structures 66 . The second assumption is a theoretical simplification and has no impact on our main results [Eqs. (5), (6) and the first two columns of Table I as will be borne out in App. C. These assumptions allow us to model the supports as elastic half-spaces.

Thus, the whole structure presents reflection symmetries with respect to the $x$ and $y$ axes that are associated, respectively, with the operators $\hat{R}_{x}$ and $\hat{R}_{y}$ acting on the space of solutions of the elastic wave equations. This allows us to find normal modes of the whole structure that are eigenvectors of $\hat{R}_{x}$ and $\hat{R}_{y}$. For a given normal mode $|u\rangle$ one can generate modes $\left|u_{++}\right\rangle,\left|u_{+-}\right\rangle,\left|u_{-+}\right\rangle$, and $\left|u_{--}\right\rangle$with the desired reflection properties by the following symmetrization procedure

$\left|u_{\mu \nu}\right\rangle=\hat{S}_{\mu \nu}|u\rangle \equiv \frac{1}{2}\left(|u\rangle+\mu \hat{R}_{x}|u\rangle+\nu \hat{R}_{y}|u\rangle+\mu \nu \hat{R}_{x} \hat{R}_{y}|u\rangle\right)$

with $\mu, \nu= \pm$, which guarantees:

$$
\begin{aligned}
& \hat{R}_{x}\left|u_{\mu \nu}\right\rangle=\mu\left|u_{\mu \nu}\right\rangle, \\
& \hat{R}_{y}\left|u_{\mu \nu}\right\rangle=\nu\left|u_{\mu \nu}\right\rangle .
\end{aligned}
$$

We note that

$$
|u\rangle=\frac{1}{2} \sum_{\mu, \nu= \pm}\left|u_{\mu \nu}\right\rangle
$$

Furthermore as in any standard scattering problem $\frac{56}{}$, we can choose for these modes $\{|u\rangle\}$ incoming scattering states that present simple asymptotic behavior for $t \rightarrow-\infty$ corresponding to the different modes that can propagate in the support(s) - i.e. the free modes of an elastic half-space ${ }^{45146}$. Thus for each type of mode with well defined reflection properties, there will be four types of asymptotic behavior, namely: longitudinal bulk waves $(l)$, transverse bulk waves polarized along the plane of incidence (SV waves), transverse bulk waves polarized perpendicular to the plane of incidence ( $\mathrm{SH}$ waves), and Rayleigh surface waves $(s)$ - also known as SAW. In all four cases the corresponding unsymmetrized solutions for a free elastic half space can be written in the form:

$$
\bar{u}_{\bar{q}, \gamma}^{(0)}(\bar{r})=\frac{1}{(2 \pi)^{d_{\gamma} / 2}}\left[\bar{\varepsilon}_{0} e^{i \bar{q} \cdot \bar{r}}+A_{l} \bar{\varepsilon}_{l} e^{i \bar{q}_{l} \cdot \bar{r}}+A_{t} \bar{\varepsilon}_{t} e^{i \bar{q}_{t} \cdot \bar{r}}\right] .
$$


where $q_{z}>0$ [cf. Fig. 1] and $\gamma=l$, SV, SH, $s$ labels the type of mode. The first term corresponds to the incident wave and the last two to the reflected longitudinal $(l)$ and transverse $(t)$ waves. The dimensionality $d_{\gamma}$, polarizations $\bar{\varepsilon}$, wavevectors $\bar{q}_{l / t}$ and amplitudes $A_{l / t}$ (which depend on $\bar{q}$ and $\gamma$ ) corresponding to each of these types of modes are given in Appendix A In addition for the bridge geometry there will be right- and left-movers and therefore eight types of modes. Thus, each mode $\bar{u}_{\bar{q}, \gamma}^{(0)}(\bar{r})$ of the support on the left with $q_{x}, q_{y}, q_{z}>0$ will generate four incoming "right-moving" symmetrized normal modes of the whole structure $\bar{u}_{\bar{q}, \gamma, \mu, \nu, \mathrm{R}}(\bar{r})$ - with analogous relations and definitions for the left-movers and in the case of a cantilever. In the following, to simplify the notation, we will absorb the discrete indices in the $q$ [i.e. $\left.\bar{u}_{\bar{q}, \gamma, \mu, \nu, \mathrm{R} / \mathrm{L}}(\bar{r}) \rightarrow \bar{u}_{q}(\bar{r})\right]$ unless otherwise stated. Of course, for the bridge geometry we can exploit the reflection symmetry with respect to the $x-y$ plane through the midpoint of the beam to obtain the left-movers from the right-movers so that only the latter need to be calculated.

On the other hand the beam can be viewed essentially as a phonon waveguide. Thus it presents a series of branches associated with the size quantization of the transverse wavevector. An ubiquitous feature of these systems is that there are four branches that lack an infrared cut-off: two bending branches (vertical and horizontal) with quadratic dispersion relations, and a torsional and a compression branch with linear dispersion relations 45146 . Each of these branches corresponds to a given type of symmetrized modes $\left|u_{\mu \nu}\right\rangle-\left|u_{++}\right\rangle$for compression (c), $\left|u_{+-}\right\rangle$for vertical bending $(v),\left|u_{-+}\right\rangle$ for horizontal bending $(h)$ and $\left|u_{--}\right\rangle$for torsion $(t)$. As we are interested only in low frequency phonons these are the only modes that can propagate inside the beam. Henceforth we will identify the discrete indices $\mu, \nu$ of the normal modes $\bar{u}_{q}(\bar{r})$ with the corresponding branch inside the beam by introducing a suitable branch index $\beta=c, t, v, h$ (i.e. $\{\mu, \nu\} \rightarrow \beta$ ). As already discussed, to describe these branches at low frequencies one can resort to TRE ${ }^{45}$, that consists of an approximation in which the small parameter is $k d$. More precisely it corresponds to taking the lowest order terms in an expansion in $k d$ of the transverse profile of the mode. The associated displacements are given in Appendix B. For a finite length beam, in addition to these propagating solutions, it is necessary to also consider the role of exponentially decaying evanescent solutions. These are of two types: end corrections that decay over a length scale of order $d$ and solutions associated with the bending branches with a decay length $1 / k$ - the latter can also be treated within TRE.

As we are interested in properties of the beam's motion our main task will be to determine $\bar{u}_{q}(\bar{r})$ inside the beam (and a distance $\gg d$ away from the junctions). This problem is analogous to the one of finding the electromagnetic field inside a Fabry-Pérot interferometer. Thus it can be approached by considering how the elastic waves that can propagate inside the beam are reflected at the abrupt junctions (and at the free end for the cantilever) and then adding the infinite reflections. In the cases of the bending branches for $k \lesssim 1 / L$ it is clear that the real exponentials are not simply end corrections and have to be taken into account in this resummation process. Hence there are two ways of propagating for the bending modes. This reduction of the problem to the reflection at a single junction is presented in Section III] In addition one needs to consider how a wave incident from the support is transmitted into the beam. These problems of reflection and transmission at a single junction are tackled in Sec. IV.

Heuristically, for very long wavelengths the abrupt junction is seen from the inside of the beam as a clamped boundary condition, and from the outside (i.e. from the support) as a free boundary condition. Let us consider for example the problem of reflection from the inside. If one adopts as an approximation for the displacements inside the beam the solution that corresponds to a clamped boundary condition $\bar{u}_{*}(\bar{r})$ it can be proved that the associated relative error is at most of order $k d$ (cf. Subsec. IV A. An analogous treatment can be done for the problem of transmission into the beam from the outside. It will be proven in Subsec. IVB that the associated transmission amplitudes can be exactly related by a reciprocity argument to the total transmission coefficient into the support $\tau(\omega)$ associated with reflection from the inside of the beam. This and the aforementioned approximation by free boundary conditions (cf. Subsec. IV A) will allow us to establish simple expressions for the functions $\tilde{\rho}(\omega)$ and $\tau(\omega)$ valid to lowest order in $k d$.

Finally, in Sec. $\mathrm{V}$ we will relate the environmental spectral density $I(\omega)$ for each resonance with the normal modes $\bar{u}_{q}(\bar{r})$ and establish the fundamental relations (5), (6) between the functions $I(\omega)$ and $\tilde{\rho}(\omega), \tau(\omega)$. As will be discussed in the following Subsection, their derivation involves the use of a canonical transformation that relates a closed quantum system "scattering representation" for the "mechanical Fabry-Pérot" (furnished by the supported beam) with an open quantum system "resonator-bath representation" that singles out one of its resonances.

\section{B. Closed system vs. open system}

To each normal mode $\bar{u}_{q}(\bar{r})$ with frequency $\omega(q)$ we can associate a bosonic annihilation operator $b(q)$. Thus, the normal ordered Hamiltonian operator for the whole structure considered as a closed quantum mechanical system adopts the simple form

$$
H=\int_{q} \hbar \omega(q) b^{\dagger}(q) b(q) .
$$

Here $\int_{q}$ denotes $\int_{0}^{\infty} \mathrm{d} q_{x} \mathrm{~d} q_{y} \mathrm{~d} q_{z}$ and summation over the appropriate discrete indices detailed in Subsec. IIA. In this diagonal representation the resonances will emerge 
as poles of the propagator for the displacement field

$$
\hat{\bar{u}}(\bar{r})=\int_{q} \sqrt{\frac{\hbar}{2 \rho_{s} \omega(q)}} \bar{u}_{q}(\bar{r}) b^{\dagger}(q)+\text { H.c. },
$$

and in this sense may be regarded as "derived objects". It is important to note that when the beam is characterized by a different density $\rho_{b} \neq \rho_{s}$ the above eigenmodes of the whole structure are orthonormal in a non-trivial metric which defines the scalar product that will be used in general (except in Sec. IV and Apps. B and C):

$$
\langle v \mid u\rangle \equiv \int \mathrm{d} r^{3} \frac{\rho(\bar{r})}{\rho_{s}} \bar{v}^{*}(\bar{r}) \cdot \bar{u}(\bar{r}) .
$$

On the other hand in the context of the applications already discussed (cf. Sec. I), where the system is driven out of equilibrium, it is normally convenient to isolate explicitly the relevant degrees of freedom of the nanoresonator and treat them as an open quantum system. In the simplest scenario there is one resonance of interest so that the goal is to find a "resonator-bath" representation in which the Hamiltonian adopts the form

$$
\begin{aligned}
H= & \hbar \omega_{R} b_{R}^{\prime \dagger} b_{R}^{\prime}+\hbar\left(b_{R}^{\prime}+b_{R}^{\prime \dagger}\right) \int_{q}\left[\zeta(q) b^{\prime}(q)+\text { H.c. }\right] \\
& +\int_{q} \hbar \omega(q) b^{\prime \dagger}(q) b^{\prime}(q),
\end{aligned}
$$

with off-diagonal couplings $\zeta(q)$ that are sufficiently weak so that the renormalization of the bare frequency $\omega_{R}$ is smaller than the characteristic spacing between the resonances - i.e. the free spectral range of the mechanical Fabry-Pérot. In such a representation the relevant resonance will correspond to the degree of freedom described by the operators $b_{R}^{\prime}, b_{R}^{\prime \dagger}$ - which annihilate or create a quanta in the resonator mode $\bar{u}_{R}^{\prime}(\bar{r})$ - and in this sense can be viewed as a "fundamental object". The discrete mode $\bar{u}_{R}^{\prime}(\bar{r})$ should be real, localized in the beam, have finite averaged elastic energy density per unit amplitude given by $\rho_{s} \omega_{R}^{2} / 2$, and satisfy the elastic wave equation inside the beam. In turn the $b^{\prime}(q)$ will be annihilation operators for a continuum of modes $\bar{u}_{q}^{\prime}(\bar{r})$ [the environment] that have support in the whole structure and are labeled after the $\bar{u}_{q}(\bar{r})$ with which they are in a one to one correspondence ${ }^{67}$. More precisely, the classical state $\bar{u}_{q}^{\prime}(\bar{r})$, evolved with the Hamiltonian density that corresponds to setting $\zeta(q)=0$ in Eq. 20), and the classical state $\bar{u}_{q}(\bar{r})$, evolved with the elastic wave equation for the whole structure, will share the same "free" asymptotic behavior for $t \rightarrow-\infty$. The form of the off-diagonal term in which the environment only couples to the canonical coordinate of the resonator $\hat{X}_{R}$ reflects the fact that the underlying phonon Hamiltonian is diagonal in the momenta. This, after integrating out the resulting equations of motion for the $b^{\prime}(q)$ allows to obtain the well known Equation $\frac{68}{11}$.

The Hamiltonian (20) is just an instance of the thoroughly studied problem of a single harmonic oscillator linearly coupled to a field 39 . The dynamics of the resonator mode is completely determined by the following environmental spectral density:

$$
I(\omega) \equiv 2 \pi \int_{q}|\zeta(q)|^{2} \delta[\omega-\omega(q)]
$$

This problem is integrable given that $H$ can be diagonalized by a linear canonical transformation $\frac{48 / 57}{15}$ that would allow to determine the $\bar{u}_{q}(\bar{r})$ given $\bar{u}_{q}^{\prime}(\bar{r}), \bar{u}_{R}^{\prime}(\bar{r}), \omega_{R}$ and $\zeta(q)$. We are simply faced with the inverse problem: the derivation of Hamiltonian 200, with $\bar{u}_{R}^{\prime}(\bar{r})$ and $\{\zeta(q)\}$ satisfying the aforementioned properties, from Hamiltonian (17) given the normal modes $\left\{\bar{u}_{q}(\bar{r})\right\}$. The solution is not unique $e^{50}$ but quantities of physical interest - e.g. the resonator's frequency dressed by the environment, its $Q$-value and its relative oscillator strength in physical spectra - will remain invariant under different choices.

Our analysis of the long wavelength behavior of the propagation of elastic waves inside the resonator (cf. Subsec. IVA and the fact that the resonances of interest have low frequencies that correspond to wavelengths much larger than $d$, prompts us to choose for $\bar{u}_{R}^{\prime}(\bar{r})$ an eigenmode of the elastic wave equation satisfying clamped-clamped (clamped-free) boundary conditions for the bridge (cantilever) geometries. It is straightforward to prove, using the expression of the elastic en-

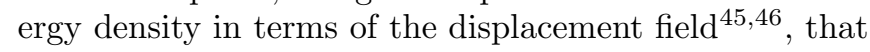
for this choice $\omega_{R}$ will be given by the corresponding eigenvalue $e^{69}$. On the other hand the diagonal representation afforded by the normal modes $\bar{u}_{q}(\bar{r})$ directly yields:

$$
\omega_{R}^{2}=\int_{q} \omega^{2}(q)\left|\left\langle u_{q} \mid u_{R}^{\prime}\right\rangle\right|^{2}
$$

The required canonical transformation from the diagonal representation (17) to the "resonator-bath" representation of choice is specified by:

$$
\begin{aligned}
b(q)= & \frac{1}{2}\left[\sqrt{\frac{\omega(q)}{\omega_{R}}}+\sqrt{\frac{\omega_{R}}{\omega(q)}}\right]\left\langle u_{q} \mid u_{R}^{\prime}\right\rangle b_{R}^{\prime} \\
& +\frac{1}{2}\left[\sqrt{\frac{\omega(q)}{\omega_{R}}}-\sqrt{\frac{\omega_{R}}{\omega(q)}}\right]\left\langle u_{q} \mid u_{R}^{\prime}\right\rangle b_{R}^{\prime \dagger} \\
& +\int_{q^{\prime}} \frac{1}{2}\left[\sqrt{\frac{\omega(q)}{\omega\left(q^{\prime}\right)}}+\sqrt{\frac{\omega\left(q^{\prime}\right)}{\omega(q)}}\right]\left\langle u_{q} \mid u_{q^{\prime}}^{\prime}\right\rangle b^{\prime}\left(q^{\prime}\right) \\
& +\int_{q^{\prime}} \frac{1}{2}\left[\sqrt{\frac{\omega(q)}{\omega\left(q^{\prime}\right)}}-\sqrt{\frac{\omega\left(q^{\prime}\right)}{\omega(q)}}\right]\left\langle u_{q} \mid u_{q^{\prime}}^{\prime *}\right\rangle b^{\prime \dagger}\left(q^{\prime}\right),
\end{aligned}
$$

and the analogous relation implied for the $b^{\dagger}(q)$, with the 
spatial overlap $\left\langle u_{q} \mid u_{q^{\prime}}^{\prime}\right\rangle$ given by:

$$
\begin{aligned}
\left\langle u_{q} \mid u_{q^{\prime}}^{\prime}\right\rangle= & \delta\left(q-q^{\prime}\right)+\frac{\left\langle u_{q} \mid u_{R}^{\prime}\right\rangle\left\langle u_{R}^{\prime} \mid u_{q^{\prime}}\right\rangle}{\Delta_{S}\left[\omega\left(q^{\prime}\right)\right]-i S\left[\omega\left(q^{\prime}\right)\right]} \\
& \times \frac{1}{\omega^{2}(q)-\omega^{2}\left(q^{\prime}\right)-i \epsilon} .
\end{aligned}
$$

Here we have defined, for any smooth function $f(\omega)$,

$$
\Delta_{f}(\omega) \equiv \frac{1}{\pi} \mathscr{P} \int_{0}^{\infty} \mathrm{d} \omega^{\prime} \frac{2 \omega^{\prime}}{\omega^{2}-\omega^{\prime 2}} f\left(\omega^{\prime}\right),
$$

and introduced the function

$$
S(\omega) \equiv \frac{\pi}{2 \omega} \int_{q}\left|\left\langle u_{R}^{\prime} \mid u_{q}\right\rangle\right|^{2} \delta[\omega-\omega(q)] .
$$

We note that this definition remains invariant if we replace the $\bar{u}_{q}(\bar{r})$ by any other basis of normal modes of the whole structure, so that the function $S(\omega)$ is a property of the resonator mode. In fact, it corresponds for $\omega>0$ to the spectral density of the resonator mode's canonical coordinate at zero temperature. The choice of $-i \epsilon$ in Eq. 24 yields the desired asymptotic condition for $t \rightarrow-\infty$, already discussed. The normalization of the resonator mode and Eq. (22) imply two sum rules

$$
\int_{0}^{\infty} \omega S(\omega) \mathrm{d} \omega=\frac{\pi}{2} \quad \text { and } \quad \int_{0}^{\infty} \omega^{3} S(\omega) \mathrm{d} \omega=\frac{\pi}{2} \omega_{R}^{2},
$$

respectively, which will prove useful below. Here we have used that for any smooth function $f(\omega)$

$$
\int_{q}\left|\left\langle u_{R}^{\prime} \mid u_{q}\right\rangle\right|^{2} f[\omega(q)]=\int_{0}^{\infty} \mathrm{d} \omega \frac{2 \omega}{\pi} S(\omega) f(\omega) .
$$

On the other hand the overlap $\left\langle u_{q} \mid u_{q^{\prime}}^{\prime *}\right\rangle=\left\langle u_{q^{\prime}}^{\prime} \mid u_{q}^{*}\right\rangle$ can be expressed in terms of $\left\langle u_{q^{\prime}}^{*} \mid u_{q}\right\rangle$ and the overlap (24) inserting $\mathbb{1}=\int_{q}\left|u_{q}\right\rangle\left\langle u_{q}\right|$. In turn, as complex conjugation corresponds to time inversion, $\left\langle u_{q^{\prime}}^{*} \mid u_{q}\right\rangle$ is just the $S$-matrix for the corresponding elastic wave classical scattering problem 56 . The facts that the $S$-matrix only mixes states with the same frequency and that the resonator mode $\bar{u}_{R}^{\prime}(\bar{r})$ is real can then be used to establish:

$$
\left[\sqrt{\frac{\omega(q)}{\omega\left(q^{\prime}\right)}}-\sqrt{\frac{\omega\left(q^{\prime}\right)}{\omega(q)}}\right]\left\langle u_{q} \mid u_{q^{\prime}}^{\prime *}\right\rangle=\left[\sqrt{\frac{\omega(q)}{\omega\left(q^{\prime}\right)}}-\sqrt{\frac{\omega\left(q^{\prime}\right)}{\omega(q)}}\right] \frac{\left\langle u_{q} \mid u_{R}^{\prime}\right\rangle\left\langle u_{R}^{\prime} \mid u_{q^{\prime}}^{*}\right\rangle}{\Delta_{S}\left[\omega\left(q^{\prime}\right)\right]+i S\left[\omega\left(q^{\prime}\right)\right]} \frac{1}{\omega^{2}(q)-\omega^{2}\left(q^{\prime}\right)+i \epsilon} .
$$

To proceed, one can first prove that the inverse transformation has analogous form given by

$$
\begin{aligned}
b_{R}^{\prime} & =\int_{q^{\prime}} \frac{1}{2}\left[\sqrt{\frac{\omega_{R}}{\omega\left(q^{\prime}\right)}}+\sqrt{\frac{\omega\left(q^{\prime}\right)}{\omega_{R}}}\right]\left\langle u_{R}^{\prime} \mid u_{q^{\prime}}\right\rangle b\left(q^{\prime}\right)+\int_{q^{\prime}} \frac{1}{2}\left[\sqrt{\frac{\omega_{R}}{\omega\left(q^{\prime}\right)}}-\sqrt{\frac{\omega\left(q^{\prime}\right)}{\omega_{R}}}\right]\left\langle u_{R}^{\prime} \mid u_{q^{\prime}}^{*}\right\rangle b^{\dagger}\left(q^{\prime}\right), \\
b^{\prime}(q) & =\int_{q^{\prime}} \frac{1}{2}\left[\sqrt{\frac{\omega(q)}{\omega\left(q^{\prime}\right)}}+\sqrt{\frac{\omega\left(q^{\prime}\right)}{\omega(q)}}\right]\left\langle u_{q}^{\prime} \mid u_{q^{\prime}}\right\rangle b\left(q^{\prime}\right)+\int_{q^{\prime}} \frac{1}{2}\left[\sqrt{\frac{\omega(q)}{\omega\left(q^{\prime}\right)}}-\sqrt{\frac{\omega\left(q^{\prime}\right)}{\omega(q)}}\right]\left\langle u_{q}^{\prime} \mid u_{q^{\prime}}^{*}\right\rangle b^{\dagger}\left(q^{\prime}\right) .
\end{aligned}
$$

We have done this by showing that substitution of Eq. 23 and its counterpart for $b^{\dagger}(q)$ into the RHS of Eq. (30) yields the identity, which reduces to a straightforward calculation with the help of Eqs. (24)-(29). Subsequently, in an analogous fashion, one can use Eq. (30) to prove that the $b^{\prime}(q), b^{\prime \dagger}(q)$ also satisfy canonical commutation relations. Then, substitution of Eq. (23) and its counterpart for $b^{\dagger}(q)$ into the diagonal Hamiltonian (17) leads to the desired "resonator-bath" form embodied in Eq. 20 with off-diagonal couplings given by:

$$
\zeta(q)=\frac{\left\langle u_{R}^{\prime} \mid u_{q}\right\rangle}{2 \sqrt{\omega_{R} \omega(q)}} \frac{1}{\Delta_{S}[\omega(q)]-i S[\omega(q)]} .
$$

Finally, substitution of this result into the definition (21) for $I(\omega)$ allows us to obtain

$$
I(\omega)=\frac{1}{\omega_{R}} \frac{S(\omega)}{\Delta_{S}^{2}(\omega)+S^{2}(\omega)}
$$

for $\omega>0$. This together with Eq. 26) reduces the derivation of the environmental spectral density to the calculation of the overlaps $\left\langle u_{R}^{\prime} \mid u_{q}\right\rangle$ and implies that it is entirely determined by the choice for the resonator mode. We note that both the $\bar{u}_{q}(\bar{r})$ that diagonalize the Hamiltonian (20) and the $\bar{u}_{q}^{\prime}(\bar{r})$ that solve the inverse problem for a given $u_{R}^{\prime}(\bar{r})$ are fixed once their asymptotic behavior is specified.

It is worth noting that within the above exact treatment the $\left\{\zeta_{q}\right\}$ describe the coupling to an environment that - barring selection rules arising from the symmetries - includes the other high- $Q$ resonances $\left\{\omega_{n}\right\}$ of the beam coupled to the supports. Thus the naive expecta- 
tion that $I(\omega)$ be smooth on the frequency scale $\omega_{R}$ will fail in a neighborhood of $\omega=\omega_{n} \neq \omega_{R}$ where the environment is structured and $I(\omega)$ may exhibit interference effects. This issue and the natural ultraviolet cutoff for $I(\omega)$, which is set by the transverse dimension $d$, will be discussed further in Sec. $\mathrm{V}$ and App. F

\section{REDUCTION TO A SINGLE JUNCTION}

It is clear from the analysis in the previous section that we will eventually need an expression for the overlaps $\left\langle u_{R}^{\prime} \mid u_{q}\right\rangle$. As $\bar{u}_{R}^{\prime}(\bar{r})$ only has support inside the beam, knowledge of the normal mode $\bar{u}_{q}(\bar{r})$ is only needed in that region. Furthermore, it is straightforward to realize that the end corrections (cf. App. B will only yield contributions to $\left\langle u_{R}^{\prime} \mid u_{q}\right\rangle$ that are higher order in $d / L$ so that we may focus on the four branches that can propagate at low frequencies. As already discussed in Subsec. IIA. our system can then be regarded as a Fabry-Pérot interferometer for elastic waves and for the bridge we may concentrate on right-movers. It proves useful to explicitly separate the transmission amplitude $t_{q}$ for propagation inside the beam that would ensue for the corresponding problem of a single junction (cf. Sec. IV); since, given our model of the supports (cf. Subsec. II A), in the limit $d / L \rightarrow 0$ the combination $\bar{u}_{q}(\bar{r}) / t_{q}$ for $\bar{r}$ inside the beam only depends on $q$ via $\omega_{q}$ and the branch index $\beta$. For each branch the latter frequency will correspond to a wavevector $k(q)$ for propagation inside the beam $\frac{70}{70}$ and, as described in App. B, the mode profile $\bar{u}_{q}(\bar{r}) / t_{q}$ can be expressed in terms of an effective one-dimensional field $\phi_{k(q), \beta}(z)$ [cf. Eq. B8] - henceforth in this Section we will omit the $q$-dependence and the index $\beta$.

In the cases of compression and torsion only travelingwave solutions intervene (cf. App. B). Therefore

$$
\phi_{k}(z)=A_{+}(k) e^{i k z}+A_{-}(k) e^{-i k z},
$$

where we take $0<z<L$, and $A_{ \pm}(k)$ are the amplitudes of the right and left moving components of $\phi_{k}$ (cf. Fig.1). In order to determine the above amplitudes we use the usual procedure for Fabry-Pérot interference, i.e. we derive the amplitudes from the infinite sum of contributions generated by consecutive reflections at the ends. We find that they obey the following linear system of self-consistent equations:

$$
\left(\begin{array}{cc}
-1 & r^{(L)} \\
r^{(R)} & -1
\end{array}\right)\left(\begin{array}{l}
A_{+} \\
A_{-}
\end{array}\right)=\left(\begin{array}{c}
-1 \\
0
\end{array}\right),
$$

where we have defined $r^{(R / L)}$ as the amplitudes for reflection at the right/left ends of the beam, and the first entry on the RHS corresponds to the displacements generated by the incoming wave from the support. This system of equations is valid for both the bridge and the cantilever. For the bridge geometry we can use the reflection symmetry with respect to the $x-y$ plane through the midpoint of the beam to prove that the reflection amplitudes are related by $r^{(R)}=r^{(L)} e^{i 2 k L}$. Furthermore, given our choice of origin, dimensional analysis implies that for a specific support material and type of beam the reflection amplitude $r^{(L)}$ is just a function of $k d$ (analogous considerations will apply below to $r_{\delta \eta}^{(L)}$, with $\delta, \eta=A, B$, and to the ratio $b$ ).

On the other hand, as discussed in Sec. IIA and App. B, in the cases of the two bending branches one needs to also consider evanescent solutions that decay over a length scale of order $1 / k$. In order to include these in our analysis, we generalize the above procedure. The functions $\phi_{k}$ corresponding to the bending modes will have four contributions with respective amplitudes: $A_{ \pm}(k)$ for propagation to the right/left, and $B_{ \pm}(k)$ for decay to the left/right. Hence we write

$$
\phi_{k}(z)=A_{+}(k) e^{i k z}+A_{-}(k) e^{-i k z}+B_{+}(k) e^{k z}+B_{-}(k) e^{-k z}
$$

In formal terms the resummation of the successive "reflections" can be viewed as an iterative procedure in which the $n$th contribution when added to the $n-1$ th matches the boundary conditions at the right/left ends for odd/even $n$ (here $n=1,2 \ldots$ with $n=0$ corresponding to the solution for a single junction). Thus, "reflection" at one end of the beam will generate both types of contributions, i.e. there are now eight reflection amplitudes. We can define, for example, $r_{A B}^{(L)}$ as the amplitude for a propagating mode to be reflected at the left junction into a decaying mode and similarly for the other amplitudes. Hence the system of self-consistent equations obeyed by the amplitudes which specify $\phi_{k}(z)$ generalizes to

$$
\left(\begin{array}{cccc}
-1 & 0 & r_{A A}^{(L)} & r_{B A}^{(L)} \\
0 & -1 & r_{A B}^{(L)} & r_{B B}^{(L)} \\
r_{A A}^{(R)} & r_{B A}^{(R)} & -1 & 0 \\
r_{A B}^{(R)} & r_{B B}^{(R)} & 0 & -1
\end{array}\right)\left(\begin{array}{l}
A_{+} \\
B_{-} \\
A_{-} \\
B_{+}
\end{array}\right)=\left(\begin{array}{c}
-1 \\
-b \\
0 \\
0
\end{array}\right)
$$

where $b$ corresponds to the decaying contribution generated by the incoming wave from the supports. More precisely it is defined as the ratio of the decaying contribution's amplitude to $t_{q}$ for the corresponding problem of transmission from the support into the beam at a single junction (see Sec. IV). For the bridge geometry we can reduce the eight reflection amplitudes to four using again the corresponding reflection symmetry. This yields $r_{A A}^{(R)}=r_{A A}^{(L)} e^{i 2 k L}, r_{A B}^{(R)}=r_{A B}^{(L)} e^{-(1-i) k L}$, $r_{B A}^{(R)}=r_{B A}^{(L)} e^{-(1-i) k L}$, and $r_{B B}^{(R)}=r_{B B}^{(L)} e^{-2 k L}$.

Finally, we note that if one allows the ratio $b$ to depend on $q$, the results of this Section for the modes $\bar{u}_{q}(\bar{r})$ inside the beam (and a distance $\gg d$ away from the junctions) are applicable to the extent that contributions of order $\exp [-L / d]$ arising from the end corrections are negligible so that Eqs. (33)-(36) are completely general for $k(q) d \ll$ 1. 


\section{TRANSMISSION THROUGH A 3D-1D JUNCTION}

\section{A. Approximation by clamped and free boundary conditions}

We now turn to the analysis of the small $k d$ behavior of the transmission amplitudes $t_{q}, b t_{q}$ and reflection amplitudes $r_{c / t}^{(L)}, r_{v / h, \delta \eta}^{(L)}$ (with $\delta, \eta=A, B$ ) that characterize a single 3D-1D abrupt elastic junction (cf. Subsec. II A. To this effect we consider incoming eigenstates that present simple asymptotic behavior for $t \rightarrow-\infty$. In the case when the incoming wave is incident from the support these eigenstates $\left|u_{q}\right\rangle$ will correspond to the different free modes of an elastic half-space $\bar{u}_{q}^{(0)}(\bar{r})$ already discussed in Section II A. Alternatively, for incidence from the beam, they will correspond to the left-movers of an infinite beam. Once again we consider modes with well defined reflection symmetries. This implies for the eigenmodes incident from the support the same labels $q, \gamma, \beta$ as for the modes of the whole structure while the modes incident from the beam are specified by $k, \beta$.

We focus on a specific eigenmode $\bar{u}(\bar{r})$ and for simplicity omit its labels. Inside the beam [i.e. for $z \geq 0$ and $(x, y)$ in a beam's cross section] we decompose $\bar{u}(\bar{r})$ into

$$
\bar{u}(\bar{r}) \equiv \Delta \bar{u}_{+}(\bar{r})+\bar{u}_{*}(\bar{r})
$$

where $\bar{u}_{*}(\bar{r})$ is an approximation to the displacement field specified by taking at $z=0$ : $(i)$ clamped boundary conditions when the incoming wave incides from the beam or $(i i)$ displacements specified by the corresponding solution for the free elastic half-space [i.e. $\bar{u}_{*}(x, y, 0)=$ $\hat{S} \bar{u}^{(0)}(x, y, 0)$ ] for incidence from the support [cf. Eq. 13) and App. A. The problem of finding $\bar{u}(\bar{r})$ inside the beam can then be formulated as an integral equation for $\Delta \bar{u}_{+}(\bar{r}):$

$$
\Delta \bar{u}_{+}(\bar{r})=\int_{S} \mathrm{~d}{r^{\prime}}^{2} \mathbf{G}\left(\bar{r}-\bar{r}^{\prime}, \omega\right) \cdot \mathbf{F} \cdot\left[\Delta \bar{u}_{+}\left(\bar{r}^{\prime}\right)+\bar{u}_{*}\left(\bar{r}^{\prime}\right)\right],
$$

where $S$ is the beam's cross section at the origin, $\bar{r}=$ $(x, y, 0) \in S$, and $z^{\prime} \geq 0$. Here $\mathbf{F}$ and $\mathbf{G}$ are second rank tensors. The former is given by the linear differential operator that maps $\bar{u}\left(\bar{r}^{\prime}\right)$ onto $\hat{z} \cdot \boldsymbol{\sigma}\left(\bar{r}^{\prime}\right)$, where $\boldsymbol{\sigma}\left(\bar{r}^{\prime}\right)$ is the induced stress tensor, and the latter by the retarded Green's function of the free elastic half-space harmonically forced at its boundary 4 . More precisely, $G_{i j}(\bar{r}, \omega)$ is defined as the $i$-th component of the outgoing displacement field generated at point $\bar{r}$ by the harmonic stress source [frequency $\omega(k)$ ] with amplitude specified at $z^{\prime}=0$ by: $\sigma_{j z}\left(x^{\prime}, y^{\prime}, 0\right)=\delta\left(x^{\prime}\right) \delta\left(y^{\prime}\right), \sigma_{l z}\left(x^{\prime}, y^{\prime}, 0\right)=0$ for $l \neq j$. In order to establish that Eq. (38) specifies the solution for $z>0$ we just need to show that the expression 37 satisfies continuity of the displacement and the stress with a solution in the half-space $(z<0)$ having the appropriate asymptotic boundary conditions. We define $\Delta \bar{u}_{-}(\bar{r})$ as the extension of the RHS of Eq. (38) for $z<0$ and arbitrary $x, y$. In case $(i)$ this function directly gives the required solution in the support, while in case $(i i)$ the latter is afforded by $\hat{S} \bar{u}^{(0)}(\bar{r})+\Delta \bar{u}_{-}(\bar{r})$. In both cases the continuity of the stress follows trivially by construction whereas Eq. (38) enforces the continuity of the displacement. It is understood that both $\Delta \bar{u}_{+}(\bar{r})$ and $\bar{u}_{*}(\bar{r})$ are linear superpositions of the low frequency harmonic solutions $\left|v_{\beta}^{(m)}(k)\right\rangle$ of a semi-infinite elastic beam (cf. App. B) with frequency $\omega(k)$ (specified by $k(q)$ for incidence from the support). Thus $\bar{u}(\bar{r})$ satisfies the elastic wave equation for $z>0$ and the only traveling wave contributing to $\Delta \bar{u}_{+}(\bar{r})$ is the right-mover corresponding to $k, \beta$. This yields

$$
\begin{aligned}
\Delta \bar{u}_{+, \beta}(\bar{r}, k, d)= & \sum_{m} c_{\beta m}(k, d) \\
& \times \bar{A}_{\beta m}\left(x, y, d, \kappa_{\beta m}\right) e^{-\kappa_{\beta m}(k, d) z}, \\
\bar{u}_{*, \beta}(\bar{r}, k, d)= & \bar{u}_{\mathrm{in}, \beta}(\bar{r}, k, d)+\sum_{m} c_{\beta m}^{(*)}(k, d) \\
& \times \bar{A}_{\beta m}\left(x, y, d, \kappa_{\beta m}\right) e^{-\kappa_{\beta m}(k, d) z} .
\end{aligned}
$$

Here we have reintroduced the "symmetry index" $\beta$ and eliminated the frequency in favor of $k$. The amplitudes in the different harmonic solutions are given by $c_{\beta, m}(k, d)$ and $c_{\beta, m}^{(*)}(k, d)$; while $\bar{A}_{\beta, m}\left[x, y, d, \kappa_{\beta, m}(k, d)\right]$ and $\kappa_{\beta, m}(k, d)$ are, respectively, the corresponding transverse profiles and complex wavevectors of the latter. The displacement field incident from the beam $\bar{u}_{\text {in }, \beta}(\bar{r}, k, d)$ vanishes for case $(i i)$ whereas for case $(i)$ it is given by $\bar{A}_{\beta, 0}[x, y, d, i k] e^{-i k z}$ for propagating modes and $\bar{A}_{v / h, 1}\left[x, y, d,-\kappa_{v / h, 1}\right] e^{\kappa_{v / h, 1} z}$ for the large decay length exponentials associated with the bending branches (decaying modes). This "incident displacement" and the terms with $m=0$ and with $\beta=v / h, m=1$ yield the TRE part of the solution, while $\beta=c, t$ with $m>0$ and $\beta=v, h$ with $m>1$ correspond to the end corrections. These are characterized by $\Re\left[\kappa_{\beta, m}(k, d)\right] \gtrsim 1 / d$ whereas

$$
\kappa_{\beta, 0}(k, d) \equiv-i k \quad \kappa_{v / h, 1}(k, d)=k(1+\mathcal{O}[k d])
$$

To extract the small $k d$ behavior of the transmission and reflection amplitudes we first prove that

$$
\frac{\left|\Delta \bar{u}_{+}(\bar{r})\right|}{|\bar{u}(\bar{r})|} \lesssim \mathcal{O}[k d] \text { for } \quad z \gg d
$$

For this analysis we eliminate $k$ and $z$ in favor of $k d$ and $k z$, respectively, which are then treated as independent variables (henceforth we omit the resulting $d$ dependence of non-dimensionless amplitudes). If one substitutes Eq. (37) into $\left|\Delta \bar{u}_{+}(\bar{r})\right| /|\bar{u}(\bar{r})|$ one can deduce that

$$
\frac{\left|\Delta \bar{u}_{+}(\bar{r})\right|}{\left|\bar{u}_{*}(\bar{r})\right|} \lesssim \mathcal{O}[k d] \Rightarrow \frac{\left|\Delta \bar{u}_{+}(\bar{r})\right|}{|\bar{u}(\bar{r})|} \lesssim \mathcal{O}[k d]
$$

so that it suffices to prove the LHS of Eq. (42). Furthermore, for $z \gg d$ the contributions of the end corrections 
are exponentially suppressed so that it suffices to analyze the TRE amplitudes [cf. Eq. [39]]. In fact it can be proved that the latter satisfy

$$
\begin{aligned}
\left|c_{\beta, m}^{(*)}(k d)\right| & \lesssim \begin{cases}\mathcal{O}[k d] & \text { for case }(i i) \text { and } \beta=t \\
\mathcal{O}[1] & \text { otherwise }\end{cases} \\
\left|c_{\beta, m}(k d)\right| & \lesssim \begin{cases}\mathcal{O}\left[(k d)^{2}\right] & \text { for case }(i i) \text { and } \beta=t \\
\mathcal{O}[k d] & \text { otherwise }\end{cases}
\end{aligned}
$$

which then directly imply the LHS of Eq. 42.

First we establish the behavior (43) of the starred amplitudes which amounts to a rigorous derivation of the recipes used in TRE to specify the boundary conditions for the effective one dimensional field $\phi(z)$. To this effect we substitute Eq. (39) into

$$
\bar{u}_{*, \beta}(x, y, 0, k, d)= \begin{cases}0 & \text { for case }(i) \\ \hat{S}_{\beta} \bar{u}_{q}^{(0)}(x, y, 0) & \text { for case }(i i)\end{cases}
$$

and take on both sides the spatial averages $\langle\ldots\rangle_{S}$ and $\langle\ldots\rangle_{\text {ang, } S}$ that correspond, respectively, to the displacement of the center of mass and the spatially averaged angle $\bar{\theta}$ [as defined in App. B. Eqs. (B11), (B13)] for the cross section $S$ (henceforth we omit the latter label). If we consider each component separately we have six equations that are linear in the amplitudes $c_{\beta, m}^{(*)}(k d)$ with inhomogeneous terms arising from $-\bar{u}_{\mathrm{in}, \beta}(x, y, 0, k, d)$ in case $(i)$ and $\hat{S}_{\beta} \bar{u}_{q}^{(0)}(x, y, 0)$ in case $(i i)$. The reflection symmetries imply that in the cases of compression and torsion, respectively, only the equation corresponding to $\left\langle u_{z}\right\rangle$ and to $\theta_{z}$ does not vanish trivially, while for vertical (horizontal) bending the same applies to the two equations provided by $\left\langle u_{x}\right\rangle\left(\left\langle u_{y}\right\rangle\right)$ and $\theta_{y}\left(\theta_{x}\right)$. In each of these equations we solve for the TRE amplitudes in terms of the end corrections and the inhomogeneous terms. The small $k d$ behavior of the resulting expressions can be extracted using the following properties of the modes' transverse profiles:

$$
\begin{aligned}
& \left\langle A_{z, c m}\right\rangle \approx \begin{cases}\frac{1}{\sqrt{2 \pi S}} & \text { for } m=0 \\
\mathcal{O}\left[(k d)^{2}\right] & \text { otherwise }\end{cases} \\
& \left\langle A_{x / y, v / h m}\right\rangle \approx \begin{cases}\frac{1}{\sqrt{2 \pi S}} & \text { for } m=0,1 \\
\mathcal{O}\left[(k d)^{4}\right] & \text { otherwise }\end{cases} \\
& \theta_{z, t m} \approx \begin{cases}\frac{1}{\sqrt{2 \pi I_{z}}} & \text { for } m=0 \\
\mathcal{O}\left[(k d)^{2}\right] & \text { otherwise }\end{cases} \\
& \frac{\theta_{y / x, v / h m}}{k d} \approx \begin{cases} \pm \frac{(i)^{m+1}}{d \sqrt{2 \pi S}} & \text { for } m=0,1 \\
\mathcal{O}\left[(k d)^{3}\right] & \text { otherwise }\end{cases} \\
& \mid\left\langle\int _ { S } \mathrm { d } { r ^ { \prime } } ^ { 2 } \mathbf { G } ( \overline { r } - \overline { r } ^ { \prime } , \omega [ k ] ) \cdot \mathbf { F } \cdot \left\{\bar{A}_{\beta, m}\left[x^{\prime}, y^{\prime}, d, \kappa_{\beta, m}(k, d)\right]\right.\right. \\
& \left.\left.\times e^{-\kappa_{\beta, m}(k, d) z^{\prime}}\right\}\right\rangle \mid \approx \begin{cases}\mathcal{O}\left[(k d)^{p_{\beta}}\right] & \text { for } \beta, m \in \mathrm{TRE} \\
\mathcal{O}\left[(k d)^{2 p_{\beta}}\right] & \text { otherwise }\end{cases}
\end{aligned}
$$

$$
\begin{aligned}
& \mid\left\langle\int _ { S } \mathrm { d } { r ^ { \prime } } ^ { 2 } \mathbf { G } ( \overline { r } - \overline { r } ^ { \prime } , \omega [ k ] ) \cdot \mathbf { F } \cdot \left\{\bar{A}_{\beta, m}\left[x^{\prime}, y^{\prime}, d, \kappa_{\beta, m}(k, d)\right]\right.\right. \\
& \left.\left.\times e^{-\kappa_{\beta, m}(k, d) z^{\prime}}\right\}\right\rangle_{\mathrm{ang}} \mid \approx \begin{cases}\mathcal{O}\left[(k d)^{p_{\beta}}\right] & \text { for } \beta, m \in \mathrm{TRE} \\
\mathcal{O}\left[(k d)^{2 p_{\beta}}\right] & \text { otherwise }\end{cases}
\end{aligned}
$$

which imply that the contributions of the end corrections scale at most as the inertia (i.e. as $\omega^{2}$ ). The above Eqs. 46 follow directly from the universal properties of the end corrections discussed in App. B (Subsection B 2 and the small $k d$ behavior of the TRE solutions 71 (cf. Subsection B 1). Thus, with the help of the Taylor expansion of $\hat{S}_{\beta} \bar{u}_{q}^{(0)}(x, y, 0)$ at the origin, Eq. 13$)$, and Eq. (B12) we obtain for compression and torsion:

$$
c_{c, 0}^{(*)}(0)= \begin{cases}-1 & \text { for case }(i) \\ 2 \sqrt{2 \pi S} u_{z, q}^{(0)}(0) & \text { for case }(i i)\end{cases}
$$

$c_{t, 0}^{(*)}(k d) \approx\left\{\begin{array}{l}-1 \\ \sqrt{2 \pi I_{z}}\left[\hat{z} \cdot \nabla \times \bar{u}_{q}^{(0)}(0)+2 \gamma_{z} u_{x y, q}^{(0)}(0)\right]\end{array}\right.$

where $\gamma_{z} \equiv\left(I_{y}-I_{x}\right) / I_{z}$ and $u_{i j}$ is the strain tensor. In the case of the bending branches for case $(i)$ we get:

$$
\begin{aligned}
& c_{v / h, 0}^{(*)}(0)= \begin{cases}i & \text { for propagating } \bar{u}_{\mathrm{in}, \beta} \\
-(1-i) & \text { for decaying } \bar{u}_{\mathrm{in}, \beta}\end{cases} \\
& c_{v / h, 1}^{(*)}(0)= \begin{cases}-(1+i) & \text { for propagating } \bar{u}_{\mathrm{in}, \beta} \\
-i & \text { for decaying } \bar{u}_{\mathrm{in}, \beta}\end{cases}
\end{aligned}
$$

while for case $(i i)$ we obtain:

$$
\left[\begin{array}{cc}
1 & 1 \\
\pm i & \mp 1
\end{array}\right] \cdot\left[\begin{array}{c}
c_{v / h, 0}^{(*)}(0) \\
c_{v / h, 1}^{(*)}(0)
\end{array}\right]=\left[\begin{array}{c}
2 \sqrt{2 \pi S} u_{x / y, q}^{(0)}(0) \\
0
\end{array}\right]
$$

where the first equation corresponds to the average displacement $\left\langle u_{x / y}\right\rangle$ and the second one to the angle $\theta_{y / x}$. The fact that the latter does not contribute to lowest order in this case is a consequence of the linear versus quadratic dispersion relations that characterize the propagation of the relevant modes $(\beta=v, h)$ in the support and the beam, respectively. Equation (49) yields

$$
c_{v / h, 0}^{(*)}(0)=\sqrt{2 \pi S}(1-i) u_{x / y, q}^{(0)}(0) \frac{c_{v / h, 1}^{(*)}(0)}{c_{v / h, 0}^{(*)}(0)}=i .
$$

An analogous procedure can be followed to derive Eq. (44). We substitute instead the decompositions (39) into Eq. (38) and now the averages $\langle\ldots\rangle_{S}$ and $\langle\ldots\rangle_{\text {ang }, S}$ yield linear equations for the un-starred amplitudes with inhomogeneous terms arising from the starred ones. Then Eq. (44) follows from Eqs. (46)-(50) completing our derivation of Eq. (41). Furnished with the 
latter it is clear that the lowest order contributions in $k d$ to the transmission amplitudes $t_{q}(k d), b(k d) t_{q}(k d)$ and reflection amplitudes $r_{c / t}^{(L)}(k d), r_{v / h, \delta \eta}^{(L)}(k d)$ can just be extracted from $\bar{u}_{*}(\bar{r})$. Thus from Eq. (47) we obtain for both compression and torsion $r^{(L)}(0)=-1$. In turn, Eqs. (48) and (50) yield for the two bending branches: $b(0)=i, r_{A A}^{(L)}(0)=i, r_{A B}^{(L)}(0)=-(1+i)$, $r_{B A}^{(L)}(0)=-(1-i)$, and $r_{B B}^{(L)}(0)=-i$. The lowest order contribution $t_{q}^{(0)}$ to each of the transmission amplitudes $t_{q}$ is provided by the corresponding approximation for the starred amplitude $c_{\beta, 0}^{(*)}(k d)$ [cf. Eqs. 477 and 50]]. The needed values at the origin of $\bar{u}_{q}^{(0)}(\bar{r})$ and its derivatives are straightforward to obtain from the expressions given in App. A. We note that the symmetries of the half-space imply $u_{y ; q, \theta, \varphi, \gamma}^{(0)}(0)=u_{x ; q, \theta, \pi / 2-\varphi, \gamma}^{(0)}(0)$.

Finally, for the cantilever geometry a procedure analogous to the above derivation of the starred amplitudes for case $(i)$ yields the standard TRE recipes for the reflection at the free end. The latter given our choice of coordinate origin lead to: $r^{(R)}(0)=e^{i 2 k L}$ for both compression and torsion, and $r_{A A}^{(R)}(0)=i e^{i 2 k L}, r_{A B}^{(R)}(0)=(1+i) e^{(i-1) k L}$, $r_{B A}^{(R)}(0)=(1-i) e^{(i-1) k L}, r_{B B}^{(R)}(0)=-i e^{-2 k L}$ for the two bending branches.

\section{B. Reciprocity relations}

Reciprocity is simply the relation $\left\langle v_{+} \mid u_{-}\right\rangle=\left\langle u_{-}^{*} \mid v_{+}^{*}\right\rangle$ for a standard scattering problem in a time reversal invariant theory $\sqrt{56158}$. Here $v \in V$ and $u \in U$ label freely propagating asymptotic states belonging to the sets of interest $V$ and $U$, "-" and "+" denote respectively incoming and outgoing scattering states and "*" denotes the time reversal operation. The inner product " $\langle\mid\rangle$ " is assumed to be preserved by the time evolution - i.e. the underlying theory is "unitary". However this preserved inner product need not necessarily be the usual overlap as in quantum mechanics where the "unitarity" corresponds to the preservation of probability. In particular in our case of elasticity theory it is defined so that $\langle w \mid w\rangle$ corresponds to the energy carried by the solution $w$ and the unitarity corresponds to energy conservation 56 . This relation is quite general but proves to be especially powerful when $V \sim U$, in the sense that aside from possible discrete indices, the available free eigenstates are essentially equivalent. In our specific context an example satisfying this last requirement would be two different rods (corresponding to $V$ and $U$ ) joined at an abrupt junction ${ }^{33}$. Reciprocity directly implies that for unit incident power in a traveling wave $\in U$ (with wave vector $k_{U}$ ) the power transmitted into a traveling wave $\in V$ (with wave vector $k_{V}$ ) is equal to the power transmitted into the wave corresponding to $-k_{U}$ for unit incident power in the one characterized by $-k_{V}$.

However our model for one junction does not satisfy the above "asymptotic equivalence" since it involves coupling a 1D system (the beam) to 3D and 2D continua corresponding, respectively, to the bulk and surface states (SAW) of the support. To overcome this difficulty we first consider a model of the junction for which: $(i)$ the support is characterized by some finite dimension $D,(i i)$ the support states under scrutiny are equivalent to a phonon waveguide so that the "asymptotic equivalence" is satisfied, and (iii) for $D \rightarrow \infty$ the support tends to a free elastic half-space. Then we apply the reciprocity relations for finite $D$ and finally take the limit $D \rightarrow \infty$. For bulk states (i.e. $q$ with $\gamma \in\{l, \mathrm{SV}, \mathrm{SH}\}$ ) a suitable "finite support" is afforded by another beam of square cross-section (side $D$ ) subject to periodic boundary conditions on the external faces. On the other hand for surface states (i.e. $\gamma=s$ ) a suitable construction is given by a slab of thickness $D$ subject to periodic boundary conditions at the external semi-infinite horizontal faces and a free boundary condition at the finite vertical face. In both cases the decoupled support is exactly solvable and the support states of interest are equivalent to a phonon waveguide whose branches we index with a single label $j$. Thus the free support eigenmodes ${ }^{72}$ read $\left|u_{j}^{(0)}\left(k^{\prime}, D\right)\right\rangle$ with dispersion relations $\omega_{j}\left(k^{\prime}, D\right)$ where $k^{\prime}$ is the wavevector along the waveguide's axis. If we define the amplitudes $t_{\beta, j}\left(k^{\prime}, D\right)$ such that

$$
\bar{u}_{j-}^{(0)}\left(k^{\prime}, D, \bar{r}\right) \longrightarrow t_{\beta, j}\left(k^{\prime}, D\right) \bar{v}_{\beta}^{(0)}\left[k_{\beta, j}\left(k^{\prime}, D\right), \bar{r}\right]
$$

asymptotically for $z \rightarrow \infty$, where $\left|v_{\beta}^{(0)}(k)\right\rangle$ are the freely propagating TRE beam modes (cf. App. B) with $k>0$ and $k_{\beta, j}\left(k^{\prime}, D\right)$ the wavevector in the beam's branch $\beta$ that corresponds to the frequency $\omega_{j}\left(k^{\prime}, D\right)$; we have

$$
\lim _{D \rightarrow \infty}\left|u_{j-}^{(0)}\left(k^{\prime}, D\right)\right\rangle=\left|u_{q}\right\rangle \Rightarrow \lim _{D \rightarrow \infty} t_{\beta, j}\left(k^{\prime}, D\right)=t_{q} .
$$

Taking into account that the modes we consider are normalized (in the standard Euclidean metric) in all 1D, 2D and $3 \mathrm{D}$ cases, it is then straightforward to realize that

$$
\begin{aligned}
\lim _{D \rightarrow \infty} \int \mathrm{d} k^{\prime} \sum_{j}\left|t_{\beta, j}\left(k^{\prime}, D\right)\right|^{2} \delta & {\left[\omega-\omega_{j}\left(k^{\prime}, D\right)\right] } \\
& =\int_{q}\left|t_{q}\right|^{2} \delta\left(\omega-\omega_{q}\right)
\end{aligned}
$$

where the $\{q\}$ run only over modes with the corresponding $\beta$ and the integration includes summation over the appropriate discrete indices. On the other hand reciprocity directly implies

$$
\tau_{\beta}^{(j)}\left[k_{\beta, j}\left(k^{\prime}, D\right), D\right]=\frac{P_{\beta}\left[\omega_{j}\left(k^{\prime}, D\right)\right]}{P_{j}\left[\omega_{j}\left(k^{\prime}, D\right), D\right]}\left|t_{\beta, j}\left(k^{\prime}, D\right)\right|^{2} .
$$

Here we have introduced $\tau_{\beta}^{(j)}(k, D)$ as the transmission coefficient into the support branch $j$ for a traveling wave of type $\beta$ incident from the beam with wavevector $-k$ and $P_{\beta}(\omega)\left[P_{j}(\omega, D)\right]$ as the power carried by the normalized 
free waveguide mode with frequency $\omega$ and branch index $\beta[j]$. The functions $P_{\beta}(\omega), P_{j}(\omega, D)$ have a universal expression in terms of the mass density of the respective waveguide and the corresponding dispersion relation. On the one hand, in complete analogy to the equivalent scenario for the electromagnetic field, the power carried by the waveguide mode is given by the product of the group velocity and the corresponding energy per unit length (averaged over a period). On the other hand the latter can be expressed in terms of the mass density and the frequency by first considering the harmonic theory of the underlying microscopic discrete lattice for a finite waveguide of length $2 \pi / k$ subject to periodic boundary conditions at its ends and then taking the continuum limit. Thus we obtain

$$
P_{\beta}(\omega)=\frac{1}{4 \pi} \rho_{b} \omega^{2} \frac{\mathrm{d} \omega_{\beta}}{\mathrm{d} k}, \quad P_{j}(\omega)=\frac{1}{4 \pi} \rho_{s} \omega^{2} \frac{\mathrm{d} \omega_{j}}{\mathrm{~d} k^{\prime}},
$$

where $\rho_{b}\left(\rho_{s}\right)$ is the density of the beam (support). We can substitute Eqs. (54) and (55) into the LHS of Eq. (53) and perform the integration, the summation and the limit to obtain for each branch $\beta$ the following reciprocity relation:

$$
\frac{\rho_{b}}{\rho_{s}} \int_{q}\left|t_{q}\right|^{2} \delta\left(\omega-\omega_{q}\right)=\tau_{\beta}\left[k_{\beta}(\omega)\right] \frac{\mathrm{d} k_{\beta}}{\mathrm{d} \omega}(\omega)
$$

where $\tau_{\beta}(k)$ is the total transmission coefficient into the support for a traveling wave of type $\beta$ incident from the beam with wavevector $-k$, and the $\{q\}$ run only over modes with the corresponding $\beta$.

Finally we note that the above derivation can be extended to the cases considered in App. $\mathrm{C}$ by suitable modifications of requirement (iii) so that in all cases the limit $D \rightarrow \infty$ yields the support under consideration ("3D asymmetric" or slab). Naturally, in the asymmetric cases the labels $q$ will no longer relate to the beam branch index $\beta$ and in Eq. (56) we will have $t_{q} \rightarrow t_{q, \beta}$ with the $\int_{q}$ running over all the support modes. In the case of a SWNT for which we use the shell "continuum" model $\rho_{b}$ should be replaced by the surface density of graphene $\sigma_{G}$, while for a nanowire or SWNT for which the underlying model for the $\left|v_{\beta}^{(0)}(k)\right\rangle$ is discrete the adequate ansatz reads: $\rho_{b} \rightarrow \mu_{b} / N_{c}$ (cf. App. C). In the case of a thin plate geometry ${ }^{33}$ both $\rho_{s}$ and $\rho_{b}$ should be replaced by the surface density. In fact it can be argued that Eq. (56) does not depend on any specific properties of the junction or the support and only relies on the phonon transport being ballistic.

\section{Transmission coefficients for each branch}

We turn now to the evaluation of the leading contribution in $k d$ to the LHS of Eq. (56) for each branch $\beta$ which we define as $\tilde{\rho}_{\beta}(\omega)$. On the one hand we will find in Subsec. VB that the force spectral densities $I_{n, \beta}(\omega)$ correct to lowest order in the reciprocal of the aspect ratio $d / L$ only depend on the amplitudes $t_{q}^{(0)}$ through these quantities $\tilde{\rho}_{\beta}(\omega)$. On the other hand it is clear that if we substitute into Eq. 56 the approximations $t_{q}^{(0)}$ given in Subsec. IV A the $\tilde{\rho}_{\beta}(\omega)$ determine the transmission coefficients $\tau_{\beta}\left[k_{\beta}(\omega)\right]$ correct to lowest order in $k d$. It is important to highlight that $\tilde{\rho}_{\beta}(\omega)$ is amenable to reduction to a property of the free support at the origin that directly relates to its DOS or, in the case of torsion, to its vacuum spectrum for the angle [as defined in App. B. cf. Eqs. [B11)- $[\mathrm{B} 13]$ ] and geometrical properties of the free beam $\left(S, I_{z}\right.$, and $\left.\gamma_{z}\right)$. In fact we can consider the displacement field operator for the free support $\hat{\bar{u}}^{(0)}(\bar{r})$ and decompose it in terms of its normal modes $\sqrt{73} \bar{u}_{q}^{(0)}(\bar{r})$. Then, the latter decomposition and Eqs. 477, (50), and (56) yield

$$
\begin{aligned}
\tilde{\rho}_{c}(\omega) & =\frac{2 \rho_{b} \omega}{\hbar} S \int_{-\infty}^{\infty} \mathrm{d} t e^{i \omega t}\left\langle\hat{u}_{z}^{(0)}(0, t) \hat{u}_{z}^{(0)}(0,0)\right\rangle \\
\tilde{\rho}_{t}(\omega) & =\frac{2 \rho_{b} \omega}{\hbar} I_{z} \int_{-\infty}^{\infty} \mathrm{d} t e^{i \omega t} \lim _{S \rightarrow 0}\left\langle\hat{\theta}_{z}^{(0)}(t) \hat{\theta}_{z}^{(0)}(0)\right\rangle \\
\tilde{\rho}_{v / h}(\omega) & =\frac{\rho_{b} \omega}{\hbar} S \int_{-\infty}^{\infty} \mathrm{d} t e^{i \omega t}\left\langle\hat{u}_{x / y}^{(0)}(0, t) \hat{u}_{x / y}^{(0)}(0,0)\right\rangle,
\end{aligned}
$$

where we use Heisenberg operators and $\langle\ldots\rangle$ denotes the vacuum expectation value $e^{74}$. This connection abets the interpretation of $\tilde{\rho}_{\beta}(\omega)$ as an effective environmental DOS set forth in the Introduction (cf. Subsec. IA). We note that the latter quantity has dimensions of linear density of states and that for compression and bending it is proportional to the DOS of the support times the area of the beam's cross section. We separate the contributions of each type of support modes $\gamma$ so that $\tilde{\rho}_{\beta}(\omega) \equiv \sum_{\gamma} \tilde{\rho}_{\beta, \gamma}(\omega)$. In turn, given the symmetrization of the modes, the wavevector integration in Eq. (56) only involves $q_{x}, q_{y}, q_{z}>0$ while the symmetries of the halfspace imply for the two bending branches $\left|t_{q, \theta, \varphi, \gamma, h}^{(0)}\right|=$ $\left|t_{q, \theta, \pi / 2-\varphi, \gamma, v}^{(0)}\right|$ - where we use spherical coordinates for the wavevector $\bar{q}$ as in App. A. One can then perform the substitutions $\omega=c_{\gamma} q, v=\cos \theta$, integrate over $\omega$ and $\varphi$, and eliminate $c_{\gamma}$ in favor of $c_{t}$ to obtain:

$$
\begin{aligned}
\tilde{\rho}_{c, \gamma}(\omega) & =\frac{\rho_{b}}{\rho_{s}} \frac{4 S}{c_{t}^{3}} \tilde{u}_{c, \gamma}(\alpha) \omega^{2} \\
\tilde{\rho}_{t, \gamma}(\omega) & =\frac{\rho_{b}}{\rho_{s}} \frac{4 I_{z}}{c_{t}^{5}}\left[\tilde{u}_{t, \gamma}^{(A)}+\gamma_{z}^{2} \tilde{u}_{t, \gamma}^{(S)}(\alpha)\right] \omega^{4} \\
\tilde{\rho}_{v / h, \gamma}(\omega) & =\frac{\rho_{b}}{\rho_{s}} \frac{2 S}{c_{t}^{3}} \tilde{u}_{v / h, \gamma}(\alpha) \omega^{2}
\end{aligned}
$$

Here we have introduced the following dimensionless constants and functions of the ratio $\alpha \equiv\left(c_{t} / c_{l}\right)^{2}=$ $\left(1-2 \sigma_{s}\right) / 2\left(1-\sigma_{s}\right)$ for the supports' material $\left(\sigma_{s}\right.$ is the corresponding Poisson ratio): 


$$
\begin{aligned}
& \tilde{u}_{c, l}(\alpha)=\frac{\alpha^{3 / 2}}{2 \pi} \int_{0}^{1} \mathrm{~d} v \frac{\left(1-2 \alpha+2 \alpha v^{2}\right)^{2} v^{2}}{\left[4 \alpha^{3 / 2} \sqrt{1-\alpha+\alpha v^{2}}\left(1-v^{2}\right) v+\left(1-2 \alpha+2 \alpha v^{2}\right)^{2}\right]^{2}} \\
& \tilde{u}_{c, \mathrm{SV}}(\alpha)=\frac{2}{\pi} \int_{0}^{\sqrt{1-\alpha}} \mathrm{d} v \frac{\left(1-\alpha-v^{2}\right)\left(1-v^{2}\right) v^{2}}{16\left(1-\alpha-v^{2}\right)\left(1-v^{2}\right) v^{2}+\left(2 v^{2}-1\right)^{4}} \\
& +\frac{2}{\pi} \int_{\sqrt{1-\alpha}}^{1} \mathrm{~d} v \frac{\left(\alpha-1+v^{2}\right)\left(1-v^{2}\right) v^{2}}{\left[4 \sqrt{\alpha-1+v^{2}}\left(1-v^{2}\right) v+\left(2 v^{2}-1\right)^{2}\right]^{2}} \\
& \tilde{u}_{c, \mathrm{SH}}=0 \quad \tilde{u}_{c, s}(\alpha)=\frac{1}{2 \xi^{3}(\alpha)}\left[\sqrt{1-\alpha \xi^{2}(\alpha)}-\frac{1-\xi^{2}(\alpha) / 2}{\sqrt{1-\xi^{2}(\alpha)}}\right]^{2} C^{2}(\alpha), \\
& \tilde{u}_{t, l}^{(A)}=\tilde{u}_{t, \mathrm{SV}}^{(A)}=\tilde{u}_{t, s}^{(A)}=0 \quad \tilde{u}_{t, \mathrm{SH}}^{(A)}=\frac{1}{12 \pi}, \\
& \tilde{u}_{t, l}^{(S)}(\alpha)=\frac{\alpha^{7 / 2}}{4 \pi} \int_{0}^{1} \mathrm{~d} v \frac{\left(1-\alpha+\alpha v^{2}\right)\left(1-v^{2}\right)^{2} v^{2}}{\left[4 \alpha^{3 / 2} \sqrt{1-\alpha+\alpha v^{2}}\left(1-v^{2}\right) v+\left(1-2 \alpha+2 \alpha v^{2}\right)^{2}\right]^{2}} \\
& \tilde{u}_{t, \mathrm{SV}}^{(S)}(\alpha)=\frac{1}{16 \pi} \int_{0}^{\sqrt{1-\alpha}} \mathrm{d} v \frac{\left(1-v^{2}\right)^{2} v^{2}\left(2 v^{2}-1\right)^{2}}{16\left(1-\alpha-v^{2}\right)\left(1-v^{2}\right)^{2} v^{2}+\left(2 v^{2}-1\right)^{4}} \\
& +\frac{1}{16 \pi} \int_{\sqrt{1-\alpha}}^{1} \mathrm{~d} v\left[\frac{\left(1-v^{2}\right) v\left(2 v^{2}-1\right)}{4 \sqrt{\alpha-1+v^{2}}\left(1-v^{2}\right) v+\left(2 v^{2}-1\right)^{2}}\right]^{2} \\
& \tilde{u}_{t, \mathrm{SH}}^{(S)}=\frac{1}{24 \pi} \quad \tilde{u}_{t, s}^{(S)}(\alpha)=\frac{1}{64 \xi(\alpha)} C^{2}(\alpha) \text {, } \\
& \tilde{u}_{v / h, l}(\alpha)=\frac{1}{4 \pi} \int_{0}^{1} \mathrm{~d} v \frac{4 \alpha^{5 / 2}\left(1-\alpha+\alpha v^{2}\right)\left(1-v^{2}\right) v^{2}}{\left[4 \alpha^{3 / 2} \sqrt{1-\alpha+\alpha v^{2}}\left(1-v^{2}\right) v+\left(1-2 \alpha+2 \alpha v^{2}\right)^{2}\right]^{2}} \\
& \tilde{u}_{v / h, \mathrm{SV}}(\alpha)=\frac{1}{4 \pi} \int_{0}^{\sqrt{1-\alpha}} \mathrm{d} v \frac{\left(2 v^{2}-1\right)^{2} v^{2}}{16\left(1-\alpha-v^{2}\right)\left(1-v^{2}\right)^{2} v^{2}+\left(2 v^{2}-1\right)^{4}} \\
& +\frac{1}{4 \pi} \int_{\sqrt{1-\alpha}}^{1} \mathrm{~d} v \frac{\left(2 v^{2}-1\right)^{2} v^{2}}{\left[4 \sqrt{\alpha-1+v^{2}}\left(1-v^{2}\right) v+\left(2 v^{2}-1\right)^{2}\right]^{2}} \\
& \tilde{u}_{v / h, \mathrm{SH}}=\frac{1}{4 \pi} \quad \tilde{u}_{v / h, s}(\alpha)=\frac{\xi(\alpha)}{16} C^{2}(\alpha),
\end{aligned}
$$

where $\xi(\alpha)$ is the ratio of the velocity of propagation for surface waves to $c_{t}$ and is a function of $\alpha$ that is always less than unity 4 . We note that for compression and bending the $t_{q}$ have a non-vanishing limit for $k \rightarrow 0$ so that the frequency dependence of the quantities $\tilde{\rho}$ follows directly from the density of states of the $3 \mathrm{D}$ support ${ }^{75}$. Whilst in the case of torsion there is an extra factor of $\omega^{2}$ given that the $t_{q}^{(0)}$ scale as the derivatives of the displacement field $\bar{u}_{q}^{(0)}$. In all cases the contribution of SV support modes has two distinct terms: one corresponding to polar angles $\theta$ below the critical angle $\arccos \sqrt{1-\alpha}$ for which there is a reflected longitudinal wave, and another corresponding to angles for which the longitudinal component is evanescent. The corresponding results for the effective environmental DOS for nanotubes and nanowires can be obtained from Eq. (58) via the ansatz $\rho_{b} S \rightarrow \mu_{b}, \rho_{b} I_{z} \rightarrow \mu_{b}\left\langle r^{2}\right\rangle_{S}$ (cf. App. C). Finally, we can use Eqs. (56), (58)-(62) and the TRE dispersion relations (4) to obtain after summing over the index $\gamma$ the following expressions for the transmission coefficients into the support

$$
\begin{aligned}
\tau_{c}(k) & =4 \tilde{u}_{c}(\alpha)\left(\frac{3-4 \alpha}{1-\alpha} \frac{E_{b}}{E_{s}}\right)^{3 / 2}\left(\frac{\rho_{s}}{\rho_{b}}\right)^{1 / 2} S k^{2}, \\
\tau_{v / h}(k) & =4 \tilde{u}_{v / h}(\alpha)\left(\frac{3-4 \alpha}{1-\alpha} \frac{E_{b} I_{y / x}}{E_{s}}\right)^{3 / 2}\left(\frac{\rho_{s}}{\rho_{b} S}\right)^{1 / 2} k^{5}, \\
\tau_{t}(k)=4 & {\left[\frac{1}{12 \pi}+\left(\frac{I_{y}-I_{x}}{I_{z}}\right)^{2} \tilde{u}_{t}^{(S)}(\alpha)\right]\left(\frac{3-4 \alpha}{1-\alpha} \frac{C}{E_{s}}\right)^{5 / 2} } \\
\times & \left(\frac{\rho_{s}}{\rho_{b} I_{z}}\right)^{3 / 2} k^{4},
\end{aligned}
$$

where we have also substituted the definition of $\gamma_{z}$, used $c_{t}=\sqrt{E_{s} / 2 \rho_{s}\left(1+\sigma_{s}\right)}$, and introduced $\tilde{u}_{\beta}(\alpha)=$ 


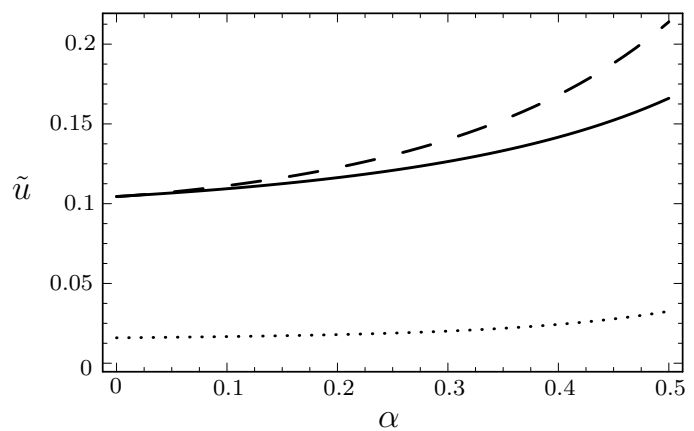

FIG. 3: Dimensionless displacements $\tilde{u}_{c}$ (dashed line), $\tilde{u}_{v / h}$ (solid line), and dimensionless angle $\tilde{u}_{t}^{(S)}$ (dotted line) as a function of the ratio $\alpha \equiv\left(c_{t} / c_{l}\right)^{2}$. For typical materials of interest $\sigma_{s} \approx 1 / 3$ which yields $\tilde{u}_{c}(1 / 4)=0.13, \tilde{u}_{t}^{(S)}(1 / 4)=$ 0.019 , and $\tilde{u}_{v / h}(1 / 4)=0.12$.

$\sum_{\gamma} \tilde{u}_{\beta, \gamma}(\alpha)$. The latter functions are plotted in Fig. 3 for all ratios $\alpha$ corresponding to physically allowed positive values of the Poisson ratio $\sigma_{s}$.

Finally, we consider the frequency dependencies and the scalings with the transverse dimensions for the analogous problem of an abrupt junction between a rectangular beam of width w and a slab (support) of the same thickness $\mathrm{t} \ll \mathrm{w} \equiv d$ [cf. App. C]. The analogous expressions for the starred amplitudes yield for the corresponding effective environmental DOS

$$
\begin{array}{lll}
\tilde{\rho}_{c}(\omega) \sim \mathrm{w} \omega & \tilde{\rho}_{t}(\omega) \sim \frac{\mathrm{w}^{3}}{\mathrm{t}^{2}} \omega \\
\tilde{\rho}_{v}(\omega) \sim \frac{\mathrm{w}}{\mathrm{t}} & \tilde{\rho}_{h}(\omega) \sim \mathrm{w} \omega
\end{array}
$$

which via Eq. (56) allow us to recover the following scalings with $k d$ for the transmission coefficients:

$$
\tau_{\beta \neq h}(k) \sim \mathrm{w} k \quad \tau_{h}(k) \sim(\mathrm{w} k)^{3}
$$

already derived in Ref. 33 by an alternative method (cf. the next Subsection). Here we have used that $\left\langle r^{2}\right\rangle_{S} \sim$ $\mathrm{w}^{2}$ and $C / I_{z} \sim \mathrm{t}^{2} / \mathrm{w}^{2}$ for $\mathrm{t} \ll \mathrm{w}$.

\section{Multipole expansion method}

The method followed above to derive the transmission coefficients relies on using the transmission amplitudes from the support into the beam - which given the results of Subsec. IVA can be extracted from the free modes of the supports - and then exploiting the reciprocity relation (56). The naive expectation is that an alternative method should be afforded by using the far field of $\Delta \bar{u}_{-}(\bar{r})[\mathrm{cf}$. Eq. (38)] corresponding to incidence from the beam [case $(i)$ in Subsec. IVA] to determine the power across the surface of a hemisphere with $r \rightarrow \infty$ per unit incident power.

The displacement field $\Delta \bar{u}_{-}(\bar{r})$ can also be viewed as the retarded solution for the problem of the free elastic half-space harmonically forced at its boundary by the stress source $\sigma_{i z}(\bar{r})=\hat{i} \cdot \mathbf{F} \cdot\left[\Delta \bar{u}_{+}(\bar{r})+\bar{u}_{*}(\bar{r})\right]$. As this radiation problem involves a source with a typical dimension $d$ much smaller than the wavelength associated with its frequency it is natural to use for $\Delta \bar{u}_{-}(\bar{r})$ its multipole expansion, whose successive moments will yield terms of increasing order in $k d$. More specifically, if we consider the asymptotic behavior of the corresponding Green's function $\frac{46}{} G_{i j}(\bar{r}, \omega)$ for $q_{t} r \rightarrow \infty$ and the $n$th moment of the stress source ${ }^{76}$

$$
M_{i_{1}, i_{2} \ldots i_{n+1}}^{(n)} \equiv S\left\langle\sigma_{i_{1} z} x_{i_{2}} x_{i_{3}} \ldots x_{i_{n+1}}\right\rangle_{S}
$$

with $i_{m}=1,2,3, n=0,1, \ldots$, one can show that the corresponding contribution to the displacement field $\Delta \bar{u}_{-}^{(n)}(\bar{r})$ scales as

$$
\left|\Delta \bar{u}_{-}^{(n)}(\bar{r})\right| \sim \frac{q_{t}^{n}(k) M^{(n)}}{E_{s} r} \quad q_{t} r \gg 1
$$

where we have introduced the norm $M^{(n)} \equiv$ $\left\{\sum_{\{i\}}\left[M_{i_{1}, i_{2} \ldots i_{n+1}}^{(n)}\right]^{2} /(n+1)\right\}^{1 / 2}$. Naturally the source $\sigma_{i z}(\bar{r})$ should also be expanded in powers of $k d$. However our treatment of the solution inside the beam (cf. Subsec. IV A only provides us with the contributions arising from the TRE part of $\bar{u}_{*}(\bar{r})$ whose order is lower than the inertia $\left[(k d)^{2 p_{\beta}}\right]$. Thus the multipole expansion will only be useful if it can be established that the former result in contributions to the transmission coefficient that dominate over those corresponding to the end corrections and to higher orders of the TRE branches. The relative orders follow directly from considering the expansion in $k d$ of the RHS of Eq. (67). A straightforward analysis implies that in general (i.e. without invoking any symmetries) this is non-trivial due $e^{77}$ to the role played by the end corrections' symmetric part of the restriction of $M_{i_{1}, i_{2}}^{(1)}$ to $i_{1}, i_{2}=1,2\left[\mathbf{M}^{(S)}\right]$ and by their moments $\mathbf{M}^{(2)}$. We note that the leading contribution to the far field of the compression, torsion, and bending TRE branches scales, respectively, as $k, k^{2}$, and $k^{3}$. In the cases of compression and bending these correspond to point sources given, respectively, by a normal force and a tangential force applied at the origin. On the other hand for torsion the leading contribution corresponds to a normal torque applied at the origin only when the corresponding $\mathbf{M}^{(S)}$ vanishes. For the bending branches the reflection symmetries are enough to cancel $\mathbf{M}^{(S)}$ and $q_{t}^{n}(k) \sim k^{2}$ implies that higher order moments are irrelevant. In the case of the torsional branch it is sufficient to have $\mathbf{M}^{(S)}=\mathbf{M}^{(2)}=0$ which can be guaranteed by also requiring symmetry under the swap $x \leftrightarrow y$ (i.e. if the cross section is symmetric under rotations by $\pi / 2$ around $z$ ). In contrast for compression augmenting the symmetry to that of a cylinder does not guarantee the cancellation of the diagonal part of $\mathbf{M}^{(S)}$ which could potentially give contributions to the far field of order $k$. Thus, whilst for bending and torsion the multipole expansion approach will provide a non-trivial corroboration of the exact form of Eqs. 63) - and together with Eqs. 587 of the reci- 
procity relation 56 - for cross sections with the appropriate symmetries, for compression it in principle only allows to check the $k$-dependence of these relations.

Energy conservation implies that the evaluation of the contribution to $\tau$ of the aforementioned leading multipole of the TRE branch can be done at the origin. In general if we consider a harmonic point source given by the superposition of a force $\bar{F}_{\text {out }}$ and a torque $\bar{M}_{\text {out }}$ applied at the origin we have for the power radiated into the half-space averaged over a period

$$
P_{\text {out }}=\frac{1}{2} \Re\left\{\bar{F}_{\text {out }}^{*} \cdot \dot{\bar{u}}_{\text {out }}(0)+\bar{M}_{\text {out }}^{*} \cdot \dot{\bar{\theta}}_{\text {out }}(0)\right\}
$$

where $\bar{\theta}_{\text {out }}(0, t)=\lim _{S \rightarrow 0}\left\langle\bar{u}_{\text {out }}(\bar{r}, t)\right\rangle_{\text {ang }, S}-$ with $S$ a circle on the free face at $z=0$ [cf. Eq. $(\bar{B} 12)]$ and $\bar{u}_{\text {out }}(\bar{r}, t)$ is the generated displacement field. If we now express the lowest order in $k d$ of the incident power associated with $\bar{u}_{\text {in }}(\bar{r}, t)[\mathrm{cf}$. Subsec. IVA] in terms of the corresponding total force $\bar{F}_{\text {in }}$ and total torque $\bar{M}_{\text {in }}$ using the TRE transverse profiles given in App. B we get

$$
\tau=\frac{P_{\text {out }}}{P_{\text {in }}}=\frac{\frac{1}{2} \Re\left\{\bar{F}_{\text {out }}^{*} \cdot \dot{\bar{u}}_{\text {out }}(0)+\bar{M}_{\text {out }}^{*} \cdot \dot{\bar{\theta}}_{\text {out }}(0)\right\}}{\frac{1}{2} \Re\left\{\bar{F}_{\text {in }}^{*} \cdot \dot{\bar{u}}_{\text {in }}(0)+\bar{M}_{\text {in }}^{*} \cdot \dot{\bar{\theta}}_{\text {in }}(0)\right\}} .
$$

Our results for the reflection coefficients $r_{c / t}^{(L)}(0)$, $r_{v / h, \delta \eta}^{(L)}(0)$ imply

$$
\left|\frac{F_{\text {out }}}{F_{\text {in }}}\right|^{2}=\left\{\begin{array}{ll}
8 & \beta=v, h \\
4 & \beta=c
\end{array} \quad\left|\frac{M_{\text {out }}}{M_{\text {in }}}\right|^{2}= \begin{cases}8 & \beta=v, h \\
4 & \beta=t\end{cases}\right.
$$

From the above Eqs. $690,(70)$, the properties of the TRE solutions and the definition of the Green's function $\mathbf{G}$ it is straightforward to establish for the leading order of the transmission coefficients

$$
\begin{aligned}
& \tau_{c}(k)=4 \Im\left\{G_{z z}[0, \omega(k)]\right\} F_{c}(k) \\
& \tau_{t}(k)=2 \Im\left\{\frac{\partial^{2}}{\partial x \partial y} G_{y x}[0, \omega(k)]-\frac{\partial^{2}}{\partial y^{2}} G_{x x}[0, \omega(k)]\right\} M_{t}(k) \\
& \tau_{v / h}(k)=4 \Im\left\{G_{x x / y y}[0, \omega(k)]\right\} F_{v / h}(k) .
\end{aligned}
$$

The last factor in each of these equations $F_{\beta}(k)\left[M_{\beta}(k)\right]$ is defined as the magnitude of the total force (torque) carried by the TRE solution per unit amplitude. To derive Eq. (71) for $\beta=t$ we have also used that the stress point source corresponding to a normal torque $M_{\text {out }}$ at the origin is specified by

$$
\sigma_{x z}=\frac{M_{\text {out }}}{2} \delta(x) \delta^{\prime}(y) \quad \sigma_{y z}=-\frac{M_{\text {out }}}{2} \delta^{\prime}(x) \delta(y) \quad \sigma_{z z}=0,
$$

which can be deduced from the source's symmetries, and the properties: $G_{x y}[x, y, z, \omega(k)]=G_{y x}[y, x, z, \omega(k)]$, $G_{y y}[x, y, z, \omega(k)]=G_{x x}[y, x, z, \omega(k)]$. Note that from the latter we also have $\Im\left\{G_{x x}[0, \omega(k)]\right\}=\Im\left\{G_{y y}[0, \omega(k)]\right\}$. If we now eliminate $k$ in favor of the transverse wavevector in the support $q_{t}$ and define

$$
\mathbf{G}\left(\bar{r}, \omega\left[q_{t}\right]\right) \equiv \frac{2\left(1+\sigma_{s}\right) q_{t}}{E_{s}} \tilde{\mathbf{G}}\left(q_{t} \bar{r}\right),
$$

dimensional analysis directly implies that the imaginary parts of the function $\tilde{\mathbf{G}}$ and of its derivatives evaluated at the origin are dimensionless functions of the Poisson ratio for the support material ${ }^{78}$. This together with

$$
F_{c}(k)=E_{b} S k \quad M_{t}(k)=C k \quad F_{v / h}(k)=E_{b} I_{y / x} k^{3}
$$

and Eqs. (71), 73 yields scalings with $k d$ for the transmission coefficients $\tau_{\beta}(k)$ that for all branches are consistent with Eqs. (63) providing a non-trivial check of the reciprocity relation (56). We have also corroborated the prefactor for torsion and bending in the cases where the aforementioned symmetries are met, by explicitly calculating $\Im\left\{\tilde{G}_{x x}(0)\right\}$ and $\Im\left\{\frac{\partial^{2}}{\partial x \partial y} \tilde{G}_{y x}(0)-\frac{\partial^{2}}{\partial y^{2}} \tilde{G}_{x x}(0)\right\} / 2$ and comparing with

$$
\begin{aligned}
\frac{1}{2} \Im\left\{\frac{\partial^{2}}{\partial x \partial y} \tilde{G}_{y x}(0)-\frac{\partial^{2}}{\partial y^{2}} \tilde{G}_{x x}(0)\right\}=\frac{1}{12 \pi} & =\tilde{u}_{t, \mathrm{SH}}^{(A)} \\
\Im\left\{\tilde{G}_{x x}(0, \alpha)\right\} & =\tilde{u}_{v / h}(\alpha),
\end{aligned}
$$

which can be obtained from Eqs. 600, 62, 63, 67, (73), (74), and the expression for $q_{t}$ in terms of $k$ for each branch. The explicit derivation of $\Im\left\{\tilde{G}_{x x}(0)\right\}$ from its definition (73) is given in App. E. We have checked numerically that the corresponding expressions for $\tilde{u}_{v / h}(\alpha)$ given, respectively, by Eqs. (E14) and (62) coincide for all physical values of the sound speed's ratio $\alpha$ corresponding to positive Poisson ratios $\left(0<\sigma_{s}<1 / 2\right)$. The analogous derivation for torsion (applicable to a cross section symmetric under rotations by $\pi / 2$ ) is greatly simplified by the fact that only $\mathrm{SH}$ waves contribute - leading to a universal prefactor. Finally, we note that Eq. 75 and its analog for compression allow us to interpret the $\tilde{u}$ as dimensionless displacements and angles.

\section{RESONATOR - BATH REPRESENTATION AND SPECTRAL DENSITIES $I(\omega)$}

\section{A. Resonator modes, scattering modes and their overlaps}

We return now to the analysis of the normal modes of the whole structure $\bar{u}_{q}(\bar{r})$ (scattering modes) and the resonator mode $\bar{u}_{R}^{\prime}(\bar{r})$ inside the beam for the purpose of determining their overlaps to lowest order in $d / L$. The prescription for the resonator mode given in Subsec. II B implies that the procedures to be followed for the bridge and cantilever geometries differ only in the boundary conditions: clamped boundary conditions at the junctions, but free boundary conditions at the end of the cantilever. Of course the resulting Sturm-Liouville problem defines an infinite set of resonator modes so that $\omega_{R} \rightarrow \omega_{n, \beta}$ with $n=0,1, \ldots$ and $\beta=c, t, v, h$. As we are interested in the regime $d / L \ll 1$, a natural requirement to identify these localized modes with the physical resonances of the whole structure is $\tilde{\omega}_{n, \beta} \rightarrow \omega_{n, \beta}$ for $d / L \rightarrow 0$, where the 
former are the real parts of the poles of the propagator for the displacement field (18) - this will be borne out below. In this respect, it is worth noting once again that our problem can be viewed as a mechanical lossy FabryPérot (cf. Subsec. II A and Sec. III). As already discussed, we focus on low frequencies so that $\omega_{\beta}[k(q)], \omega_{n, \beta} \ll \omega_{*}^{(\beta)}$ with $k_{\beta}\left(\omega_{*}\right) \sim \pi / d$. In this regime the localized resonator modes can be associated with effective one-dimensional fields $\phi_{n, \beta}(z)$ defined in complete analogy to the fields $\phi_{k(q), \beta}(z)$ describing the scattering modes [cf. Eqs. (33), (35) and Subsec. B 1]. As expected, the reflection symmetries imply that resonator modes associated with a given branch $\beta$ are orthogonal to scattering modes characterized by $\beta^{\prime} \neq \beta$.

We now consider the results for the reflection amplitudes $r_{c / t}^{(L)}(0), r_{v / h, \delta \eta}^{(L)}(0), r_{c / t}^{(R)}(0), r_{v / h, \delta \eta}^{(R)}(0)$ and for the ratio $b(0)$ obtained in the previous Section (cf. Subsec. IV A together with the analysis for the propagation of low frequency modes inside the beam performed in Sec. III [cf. Eqs. (34), (36)]. It follows that to zeroth order in $d / L$ the scattering effective one-dimensional field $\phi_{k(q), \beta}(z)$ and its derivatives satisfy the same homogeneous boundary conditions as those defining the $\phi_{n, \beta}(z)$ except for the value of the field at the left junction ${ }^{79}$ specified by

$$
\phi_{k, c / t}(0)=1 \quad \phi_{k, v / h}(0)=1+i .
$$

On the other hand all of these effective fields are solutions of the TRE equation

$$
\mathscr{D}_{\beta} \phi=\omega^{2} \phi
$$

with

$$
\mathscr{D}_{\beta} \equiv(-1)^{p_{\beta}} \tilde{c}_{\beta}^{2} \frac{\partial^{2 p_{\beta}}}{\partial z^{2 p_{\beta}}} .
$$

It is then simple to use the aforementioned boundary conditions and Eqs. (77), 78 to obtain

$$
\begin{aligned}
& \left\langle\phi_{n, \beta}\left|\mathscr{D}_{\beta}\right| \phi_{k, \beta}\right\rangle=\omega_{\beta}^{2}(k)\left\langle\phi_{n, \beta} \mid \phi_{k, \beta}\right\rangle=\omega_{n, \beta}^{2}\left\langle\phi_{n, \beta} \mid \phi_{k, \beta}\right\rangle \\
& +\left.(-1)^{p_{\beta}} \tilde{c}_{\beta}^{2} \frac{\partial^{2 p_{\beta}-1} \phi_{n, \beta}}{\partial z^{2 p_{\beta}-1}} \phi_{k, \beta}\right|_{z=0}
\end{aligned}
$$

where we have used integration by parts and that the $\phi_{n, \beta}(z)$ are real (cf. Subsec. IIB) to establish the last equality. We have calculated the resonator modes $\phi_{n, \beta}(z)$ (cf. Ref. 946) normalized them to the length $L$ and computed their necessary derivatives [note the $\pi$ phase freedom for the choice of $\left.\phi_{n, \beta}(z)\right]$. These together with Eqs. (76), (79) and the dispersion relations for the different branches, yield

$$
\left\langle\phi_{n, \beta} \mid \phi_{k(\omega), \beta}\right\rangle=\sqrt{2 C_{n, \beta}} \frac{p_{\beta}}{k_{n}} \frac{\omega_{n}^{2}}{\omega^{2}-\omega_{n}^{2}} \times \begin{cases}1 & \beta=c, t \\ e^{i \pi / 4} & \beta=v, h\end{cases}
$$

Here $n=0,1, \ldots, C_{n, c / t}=1, C_{n, v / h}=\left(\tanh ^{2} \frac{k_{n} L}{2}\right)^{(-1)^{n}}$, and $p_{\beta}$ is the exponent of the corresponding dispersion relation - i.e. $p_{c / t}=1$ and $p_{v / h}=2$.
Equation 80 will prove useful below when using the overlaps to calculate the force spectral densities $I(\omega)_{n, \beta}$. Naturally, its above derivation is invalid whenever $k$ is a zero of the resolvent of the linear system - Eq. (34) [Eq. (36)] for $\beta=c, t[\beta=v, h]$ — that determines the scattering mode $\phi_{k, \beta}(z)$ which for $k=k_{n}$ (at a generic value of $z$ ) diverges as $d / L \rightarrow 0$. The behavior of the overlaps and the spectral densities in the neighborhood of these special points, which correspond to the resonances, is discussed further in App. F Naturally the divergent behavior at $\omega_{n}$ can be used to prove $\tilde{\omega}_{n} \rightarrow \omega_{n}$.

\section{B. Relations between $I(\omega)$ and $\tilde{\rho}(\omega), \tau(\omega)$}

In principle the overlaps calculated in the previous Subsection would allow to obtain the leading contribution in $d / L$ to the environmental spectral densities $I(\omega)$ from Eqs. (26) and (32). However the latter exact expression has the drawback that even for a generic value of $\omega$ the dispersive contribution $\Delta_{S}(\omega)$ brings into play both the behavior at $\omega_{R}$ and at high frequency of the function $S(\omega)$, whilst the analysis we have done of the scattering modes $\bar{u}_{q}(\bar{r})$ and their overlaps with the resonator modes fails at these frequencies. To overcome this issue we first invert Eq. 32 to recover a well known expression for the function $S(\omega)$ in terms of $I(\omega)$

$$
S(\omega)=\frac{\omega_{R} I(\omega)}{\left[\omega^{2}-\omega_{R}^{2}-\omega_{R} \Delta_{I}(\omega)\right]^{2}+\omega_{R}^{2} I^{2}(\omega)},
$$

and subsequently derive from it the following approximate relation

$$
I(\omega) \approx \frac{S(\omega)}{\omega_{R}}\left[\left(\omega+\omega_{R}\right)^{2}\left(\omega-\tilde{\omega}_{R}\right)^{2}+\omega_{R}^{2} I^{2}\left(\tilde{\omega}_{R}\right)\right] .
$$

Here $\tilde{\omega}_{R}$ is the approximation to the renormalized frequency afforded by the solution that the equation

$$
\frac{\tilde{\omega}_{R}}{\omega_{R}}=1+\frac{\Delta_{I}\left(\tilde{\omega}_{R}\right)}{\tilde{\omega}_{R}+\omega_{R}}
$$

has close to $\omega_{R}$ when $\left|\Delta_{I}\left(\omega_{R}\right)\right| / \omega_{R} \ll 1$. The latter condition is necessary for the validity of the approximate relation 82 and the corresponding relative error is at most of order $\left|\Delta_{I}\left(\omega_{R}\right)\right| / \omega_{R}$. The derivation of Eqs. (81), $(82)$ is given in App. F where we also establish that $\left|\Delta_{I}\left(\omega_{R}\right)\right| / \omega_{R} \rightarrow 0, I\left(\omega_{R}\right) / \omega_{R} \rightarrow 0$ for $d / L \rightarrow 0$ and give the analog of the approximation 82 for the couplings $\left\{\zeta_{q}\right\}$. Hence, to obtain $I(\omega)$ correct to lowest order in $d / L$ we can replace the factor in square brackets in Eq. 82 by its limit for $d / L \rightarrow 0$ which will be given by $\tilde{\omega}_{R} \rightarrow \omega_{R}, I\left(\omega_{R}\right) \rightarrow 0$. If we now consider the definitions of $t_{q}$ and $\phi_{k}(z)$, and the corresponding expressions for the displacement field given in Sec. III] and substitute into the definition (26) we obtain for the remaining factor

$$
\frac{S(\omega)}{\omega_{R}} \approx \frac{\delta\left|\left\langle\phi_{R} \mid \phi_{k(\omega)}\right\rangle\right|^{2}}{4 L \omega_{R} \omega} \frac{\rho_{b}}{\rho_{s}} \int_{q}\left|t_{q}\right|^{2} \delta\left(\omega-\omega_{q}\right),
$$


where we have used the definition (19) for the overlap $\left\langle u_{R}^{\prime} \mid u_{q}\right\rangle$, the expressions for $\bar{u}(\bar{r})$ in terms of $\phi(z)$ given in App. B and neglected the contributions that involve derivatives of $\phi(z)$ which are higher order in $d / L$ - note that $\phi_{n} \rightarrow \phi_{R}$, defined in the previous subsection, is normalized to the length while $\left|u_{R}^{\prime}\right\rangle$ is normalized in the metric defined by Eq. (19). Here $q$ runs only over modes with the branch index $\beta$ corresponding to the appropriate resonator mode (frequency $\omega_{R}$ ) and we have introduced the number of supports $\delta$ so that for the bridge geometry $q$ is further restricted to right-movers. On the one hand the last factor on the RHS of Eq. 84, correct to lowest order in $k d$, is just given by the appropriate $\tilde{\rho}_{\beta}(\omega)$ [Eq. (58) summed over all $\gamma$ ] which together with Eq. (80) for the overlaps and the relation

$$
\left(\frac{p_{\beta} \omega_{n}}{k_{n}}\right)^{2} \frac{\mathrm{d} k_{\beta}}{\mathrm{d} \omega}(\omega)=\left(\frac{\omega_{n}}{\omega}\right)^{p_{\beta}-1} \frac{\mathrm{d} \omega_{\beta}}{\mathrm{d} k}(\omega),
$$

valid for $p_{\beta}=1,2$, yields Eq. (5), namely

$$
I_{n, \beta}(\omega)=\delta C_{n, \beta}\left[\frac{\mathrm{d} \omega_{\beta}}{\mathrm{d} k}\left(\omega_{n}\right)\right]^{2} \frac{\tilde{\rho}_{\beta}(\omega)}{2 L} \frac{\omega_{n}}{\omega} .
$$

On the other hand the last factor on the RHS of Eq. (84) can also be expressed exactly in terms of the transmission coefficients $\tau_{\beta}(\omega)$ using the reciprocity relation (56) derived in Subsec. IV B. We can use this fact, Eq. (80) for the overlaps, and Eqs. (85), (84), (82), to finally obtain the relation (6) between the leading contribution to $I(\omega)$ for each resonance and the transmission coefficients:

$$
I_{n, \beta}(\omega)=\delta C_{n, \beta} \frac{\mathrm{d} \omega_{\beta}}{\mathrm{d} k}(\omega) \frac{\tau_{\beta}(\omega)}{2 L}\left(\frac{\omega_{n}}{\omega}\right)^{p_{\beta}} .
$$

The validity of Eqs. (5) and (6), to lowest order in $d / L$ is affected by the caveats discussed in the previous Subsection, when deriving the overlaps, so that the neighborhood of the other resonances is excluded and $\omega$ should be much smaller than $\omega_{\beta}(\pi / d)$. Note that with the judicious choice of dimensionless variables $d / L, \omega / \omega_{n}$ the latter is not an additional approximation as $\omega_{\beta}(\pi / d) / \omega_{n} \rightarrow \infty$ for $d / L \rightarrow 0$. Clearly, the above procedure is questionable in a neighborhood of $\omega_{R}$ where Eq. 80 for the overlaps diverges. Nonetheless, $\omega_{R}$ is in fact included in the frequency range where Eqs. (5) and (6), are valid. To resolve this issue it suffices to prove, without using the Eqs. (81), (82), that the function $I(\omega)$ remains well behaved at this resonant frequency as $d / L \rightarrow 0$ (cf. App. F).

In turn, it can be argued that $\omega_{\beta}(\pi / d)$ always sets the order of the natural ultraviolet cutoff. If we consider the exact Eqs. (26) and (32) it is clear that the lattice constant $a$ provides an obvious ultraviolet cutoff for the functions $S(\omega)$ and $I(\omega)$ so that the case $d \sim a$ follows trivially. If instead $d \gg a$, one finds that $\omega_{\beta}(\pi / d)$ provides a "soft cutoff" beyond which the functions $S(\omega)$ and $I(\omega)$ decay as integer power-laws. In fact for $\omega \gg \omega_{\beta}(\pi / d)$ one can argue that to analyze the scaling of $S(\omega)$ it is permissible to replace in its definition $(26)$ the relevant modes $\bar{u}_{q}(\bar{r})$ inside the beam - i.e. those with $\omega_{q} \gg \omega_{\beta}(\pi / d)$ by the normal modes the beam would present for $d \rightarrow \infty$. Thus the function $\omega S(\omega)$ will scale as the DOS times the corresponding Fourier transform of the resonator mode $e^{46}$. The latter yields a factor of $1 / \omega^{2}$ for the longitudinal direction and a factor of $1 / \omega$ for each transverse direction. Hence we obtain $S(\omega) \sim 1 / \omega^{m}$, where $m=7$ for the case of a "bulk cross section" and $m=6$ for the case of a SWNT modeled as a shell52. A straightforward analysis yields in both cases $1 / \omega^{2}$ for the leading term of the asymptotic expansion of $\Delta_{S}(\omega)$. The latter behavior together with Eq. 32 leads to $I(\omega) \sim 1 / \omega^{l}$ with $l=3$ and $l=2$, respectively. Finally, it is worth noting that the scaling for $\omega \rightarrow 0$ can also be analyzed without resorting to the approximation (82). In fact one always has $\Delta_{S}(0)<0$ which together with Eqs. (84), (80), (58) and (32) imply that the functions $S(\omega)$ and $I(\omega)$ share the same scaling as a positive power-law detailed in the introduction (Subsec. IA) for the different branches.

\section{CONCLUSIONS}

In summary, we have analyzed a generic beam geometry suspended from structureless supports in the limit of large aspect ratio $d / L \rightarrow 0$ and provided for each of its low frequency resonances a Caldeira-Leggett model adequate to describe the associated quantum Brownian motion induced by phonon tunneling losses. The corresponding effective Hamiltonian for the low frequency vibrational degrees of freedom is derived from the underlying microscopic physics performing a controlled approximation in the natural small parameter $d / L$. This yields the lowest order contribution in the aspect ratio to the associated environmental force spectral densities. We find two general formulas for these functions Eq. (6) and Eq. (5) that involve, respectively, the transmission coefficient at a single junction and an effective environmental DOS. Whence providing two alternative pictures for this dissipation mechanism in terms, respectively, of phonon tunneling losses and support-induced modification of the DOS [cf. Eqs. (81), (5), and (58)]. These yield for the spectral densities functions of frequency that only depend on the length $L$ and on properties of the "decoupled" support and of the decoupled infinite beam. Furthermore they are universal in the specific sense that they only depend on the properties of the beam through the quantities that determine its low frequency effective theory known as TRE.

These environmental spectral densities result in fundamental limits for the intrinsic dissipation (i.e. upper bounds for the $Q$-values) with structureless supports which are relevant for state of the art mechanical resonators in the $0.1-1 \mathrm{GHz}$ frequency range (cf. Table $\mathrm{I}$ and Ref. 3). It is important to note the fast degradation as the length is shortened and that the fundamental limit of torsional resonators greatly exceeds that of flexural ones (for comparable frequencies ${ }^{80}$ ). In fact, for 
torsion this dissipation mechanism is likely to be negligible when compared with other contributions to the mechanical damping ${ }^{3}$. In addition we find that semiconducting SWNTs are far more resilient to this dissipation mechanism than semiconductor heterostructure realizations (typical values for the figure of merit $k_{B} T / \hbar \omega_{R} Q$ are at least an order of magnitude larger).

In the case of 3D supports the environmental spectral densities are ohmic for flexural and compressional resonances and superohmic for torsional resonances, while in the case of $2 \mathrm{D}$ slab supports they yield $1 / f$ noise with an infrared cutoff provided by the size of the slab. It is worth noting that this type of noise is normally associated with interactions involving charge degrees of freedom ${ }^{54 \mid 55}$ while here it arises in an insulator from purely vibrational effects.

Naturally, supports can be engineered to suppress the phonon tunneling losses. However in many feasible alternatives analysis of phonon propagation in a beam geometry is essential to determine the improved limits attainable ${ }^{59}$. In this context and also when analyzing situations where the resonator mode couples to a pseudospin the resonator-bath representation given in this paper may need to be complemented with an approximation for the complete phonon propagator inside the beam. In other words the effect of the "environment" cannot be "lumped" into the finite mechanical $Q$-value and there may be interference effects between different resonances arising from correlations between their effective noise sources. Clearly, away from the resonances an adequate approximation for the complete phonon propagator is afforded by the lowest order contributions in $d / L$ to the scattering modes [cf. Eq (18)]. The latter are specified by Eqs. (33)-(36) and our approximations for the reflection and transmission amplitudes given in Sec. IV - of particular interest is the behavior that results for $\omega \rightarrow 0$. On the other hand a satisfactory solution for all frequencies requires going beyond the lowest order for the reflection amplitudes - a matter pursued in detail elsewhere ${ }^{60}$.

Furthermore, we highlight the relevance of the precise connection, given in App. B, between the effective one dimensional TRE description and the underlying transverse mode profile to scenarios where the resonator is manipulated by coupling to an embedded optical emitter 20 .

Finally, we have provided a general expression for the spectral density of a given resonance in terms of the relevant elastic modes [cf. Eq. 10p] that allows to extend the treatment to other geometries. We have illustrated this for an axially symmetric resonator supported by a vertical pedestal of length at least comparable to the resonator's size. A scenario which is relevant for optomechanical systems based on microtoroids, microdisks or microspheres.

\section{Acknowledgments}

The author thanks W. Zwerger for invaluable help and thorough revision of the manuscript and acknowledges helpful discussions with C.S. Peça, A. Imamoğlu, A.N. Cleland, P. Zoller, P. Tamborenea, C. Tejedor, T.J. Kippenberg, and E. Weig.

\section{APPENDIX A: NORMAL MODES OF THE FREE ELASTIC HALF-SPACE}

The free elastic half-space supports four types of normal modes that can be classified based on the character of the incident wave: longitudinal bulk waves, transverse bulk waves with two polarizations ( $\mathrm{SV}$ and $\mathrm{SH}$ ), and Rayleigh surface waves 45146 . In all four cases the corresponding eigenfunctions can be written in the general form:

$$
\bar{u}_{\bar{q}, \gamma}^{(0)}(\bar{r})=\frac{1}{(2 \pi)^{d_{\gamma} / 2}}\left[\bar{\varepsilon}_{0} e^{i \overline{\bar{q}} \cdot \bar{r}}+A_{l} \bar{\varepsilon}_{l} e^{i \bar{q}_{l} \cdot \bar{r}}+A_{t} \bar{\varepsilon}_{t} e^{i \bar{q}_{t} \cdot \bar{r}}\right],
$$

where $d_{\gamma}$ is the dimensionality $\left(d_{\gamma}=2\right.$ for $\gamma=s$ and $d_{\gamma}=3$ for $\left.\gamma \neq s\right), \bar{\varepsilon}_{0}$ and $\bar{q}$ correspond to the polarization and wavevector of the incident wave, and $A_{t / l}, \bar{\varepsilon}_{t / l}$ and $\bar{q}_{t / l}$ to the amplitude, polarization and wavevectors of the reflected transverse/longitudinal waves. These depend on $\bar{q}$ and $\gamma$ and are given by

\section{longitudinal waves:}

$$
\begin{aligned}
\bar{q}= & \rho(\sin \theta \cos \varphi, \sin \theta \sin \varphi, \cos \theta), \\
\bar{q}_{l}= & \rho(\sin \theta \cos \varphi, \sin \theta \sin \varphi,-\cos \theta), \\
\bar{q}_{t}= & \rho\left(\sin \theta \cos \varphi, \sin \theta \sin \varphi,-\sqrt{1 / \alpha-\sin ^{2} \theta}\right), \\
\bar{\varepsilon}_{0}= & \frac{\bar{q}}{\rho}, \quad \bar{\varepsilon}_{l}=\frac{\bar{q}_{l}}{\rho}, \\
\bar{\varepsilon}_{t}= & \left(-\cos \varphi \sqrt{1-\alpha \sin ^{2} \theta},-\sin \varphi \sqrt{1-\alpha \sin ^{2} \theta},\right. \\
& -\sqrt{\alpha} \sin \theta), \\
A_{l}= & \frac{2 \alpha^{3 / 2} \sin \theta \sqrt{1-\alpha \sin ^{2} \theta} \sin 2 \theta-\left(1-2 \alpha \sin ^{2} \theta\right)^{2}}{2 \alpha^{3 / 2} \sin \theta \sqrt{1-\alpha \sin ^{2} \theta} \sin 2 \theta+\left(1-2 \alpha \sin ^{2} \theta\right)^{2}}, \\
A_{t}= & \frac{2 \sqrt{\alpha} \sin _{2} \theta\left(2 \alpha \sin ^{2} \theta-1\right)}{2 \alpha^{3 / 2} \sin \theta \sqrt{1-\alpha \sin ^{2} \theta} \sin 2 \theta+\left(1-2 \alpha \sin ^{2} \theta\right)^{2}} ;
\end{aligned}
$$

\section{SV waves:}

$$
\begin{aligned}
\bar{q} & =\rho(\sin \theta \cos \varphi, \sin \theta \sin \varphi, \cos \theta), \\
\bar{q}_{l} & =\rho\left(\sin \theta \cos \varphi, \sin \theta \sin \varphi,-\sqrt{\alpha-\sin ^{2} \theta+i \epsilon}\right), \\
\bar{q}_{t} & =\rho(\sin \theta \cos \varphi, \sin \theta \sin \varphi,-\cos \theta),
\end{aligned}
$$




$$
\begin{aligned}
\bar{\varepsilon}_{0} & =(\cos \theta \cos \varphi, \cos \theta \sin \varphi,-\sin \theta) \\
\bar{\varepsilon}_{l} & =\frac{\bar{q}_{l}}{\rho \sqrt{\alpha}}, \\
\bar{\varepsilon}_{t} & =-(\cos \theta \cos \varphi, \cos \theta \sin \varphi, \sin \theta) \\
A_{t} & =\frac{2 \sqrt{\alpha-\sin ^{2} \theta+i \epsilon} \sin \theta \sin 2 \theta-\cos ^{2} 2 \theta}{2 \sqrt{\alpha-\sin ^{2} \theta+i \epsilon} \sin \theta \sin 2 \theta+\cos ^{2} 2 \theta} \\
A_{l} & =\frac{2 \sqrt{\alpha} \sin 2 \theta \cos 2 \theta}{2 \sqrt{\alpha-\sin ^{2} \theta+i \epsilon} \sin \theta \sin 2 \theta+\cos ^{2} 2 \theta}
\end{aligned}
$$

\section{SH waves:}

$$
\begin{aligned}
\bar{q} & =\rho(\sin \theta \cos \varphi, \sin \theta \sin \varphi, \cos \theta), \\
\bar{q}_{t} & =\rho(\sin \theta \cos \varphi, \sin \theta \sin \varphi,-\cos \theta), \\
\bar{\varepsilon}_{0} & =\bar{\varepsilon}_{t}=(\sin \varphi,-\cos \varphi, 0), \\
\bar{\varepsilon}_{l} & =0, \quad A_{l}=0, \quad A_{t}=1 ;
\end{aligned}
$$

\section{surface waves:}

$$
\begin{aligned}
\bar{q} & =\rho(\cos \varphi, \sin \varphi) \\
\bar{q}_{l} & =\rho\left(\cos \varphi, \sin \varphi,-i \sqrt{1-\alpha \xi^{2}}\right), \quad(\mathrm{A} 5 \mathrm{a}) \\
\bar{q}_{t} & =\rho\left(\cos \varphi, \sin \varphi,-i \sqrt{1-\xi^{2}}\right), \quad(\mathrm{A} 5 \mathrm{~b}) \\
\bar{\varepsilon}_{0}= & 0, \quad \bar{\varepsilon}_{l}=\frac{\bar{q}_{l}}{\rho \xi \sqrt{\alpha}}, \\
\bar{\varepsilon}_{t}= & \frac{1}{\xi}\left(i \sqrt{1-\xi^{2}} \cos \varphi, i \sqrt{1-\xi^{2}} \sin \varphi, 1\right), \quad(\mathrm{A} 5 \mathrm{e}) \\
A_{l}= & -\xi \sqrt{2 \alpha \rho} C(\alpha), \quad A_{t}=i \xi \sqrt{2 \rho} \frac{\xi^{2} / 2-1}{\sqrt{1-\xi^{2}}} C(\alpha), \\
C(\alpha)= & {\left[\frac{2-\xi^{2}(\alpha)}{\left[1-\xi^{2}(\alpha)\right]^{3 / 2}}\left[\xi^{4}(\alpha) / 4+\xi^{2}(\alpha)-1\right]\right.} \\
& \left.+\frac{2-\alpha \xi^{2}(\alpha)}{\sqrt{1-\alpha \xi^{2}(\alpha)}}\right]^{-1 / 2} .
\end{aligned}
$$

Here we have defined $\sqrt{\alpha} \equiv c_{t} / c_{l}$, where $c_{t}$ and $c_{l}$ are the velocities of propagation of transverse and longitudinal waves in the elastic medium, and adopted spherical coordinates for the wavevector $\bar{q}$ (cf. Fig. 1). The parameter $\xi$ is the ratio of the velocity of propagation for surface waves to $c_{t}$ and is a function of $\alpha$ that is always less than unity 45 . We note that for SV waves with polar angles $\theta>\cos ^{-1} \sqrt{1-\alpha}$ the longitudinal component is evanescent.

\section{APPENDIX B: NORMAL MODES OF THE BEAM}

\section{TRE solutions}

At low frequencies there are four branches of propagating modes in an infinite beam: two bending branches (vertical and horizontal), a torsional and a compressional branch. An heuristic way of understanding how these four types of motion arise is to decompose the associated displacements of the points of each cross section into an overall translation, an overall rotation, and a "residual" deformation. The vicinity of free boundary conditions and the fact that the wavelength $2 \pi / k$ of these low frequency modes is much longer than $d$ determine that the deformation is higher order in the small parameter $k d$, when compared with the rotation and translation. Thus each cross section can be seen as a slightly deformed rigid body. Clearly if we considered a chain of coupled rigid bodies, each unit would have six degrees of freedom that would lead to six branches. However only four of them will have a vanishing dispersion relation and respect the requirement that the cross sections remain only slightly deformed as they move together, namely, those associated with the three possible translations and to rotation around the beam's axes. As these considerations are quite general, though throughout this Section we focus on the case when the low frequency effective theory can be derived from 3D elasticity [cf. Eq. (4)], in the case of nanowires and SWNTs the expressions for the TRE modes that follow are warranted up to the highest order for which the strain vanishes - i.e. first order for bending and zeroth order for compression and torsion - with discrete coordinates $x, y$ and up to a prefactor.

We turn now to the analysis of the small $k d$ behavior of the corresponding normalized eigenmodes for these four propagating branches $(\beta=c, t, v, h$ with $m=0)$. We may focus on a right mover given by $\left|v_{\beta}^{(0)}(k)\right\rangle \equiv$ $\bar{A}_{\beta, 0}[x, y, d,-i k] e^{i k z}$ with eigenvalue $\omega_{\beta, 0}^{2}(k, d)$ and obtain the left movers by reflection symmetry and (in the case of bending) the large decay length exponentials $\bar{A}_{v / h, 1}$ by analytic continuation. The problem of determining the Taylor expansions of $\bar{A}_{\beta, 0}$ and $\omega_{\beta, 0}^{2}(k, d)$ in powers of $k$ can be formulated as the search for an harmonic solution $\bar{u}_{\beta}(\bar{r}, t)$ of the $3 \mathrm{D}$ elastic wave equations for the beam ${ }^{45 \mid 46}$ via the ansatz:

$$
\bar{u}_{\beta}(\bar{r}, t)=\sum_{n=0}^{\infty} \bar{A}_{\beta, 0}^{(n)}(x, y) \frac{\partial^{n} \phi}{\partial z^{n}}(z, t),
$$

with $\phi(z, t)=e^{i(k z-\omega t)}$. If one neglects in Eq. (B1) all orders that scale at least as the inertia (i.e. as $\omega^{2}$ ) one obtains the TRE low frequency effective theory $45 \mid 46$. Then the function $\phi(z, t)$ will correspond to the effective one dimensional field. On the other hand, the lowest order for $\omega^{2}$ yields the corresponding approximate dispersion relation $\omega \propto k^{p_{\beta}}$. It is straightforward to realize by substitution of Eq. (B1) into the elastic wave equations for the beam $\frac{45 \mid 46}{4}$ that the result of this truncation at order $2 p_{\beta}-1$ will yield an exact solution of the corresponding static equations $(\omega \rightarrow 0)$ provided that $\frac{\partial^{2 p_{\beta}} \phi}{\partial z^{2 p_{\beta}}}$ vanishes. Thus the leading terms of the Taylor expansions of the transverse profiles $\bar{A}_{\beta, 0}$ can be extracted from the corresponding static solutions. These are given by 


\section{compression:}

$$
\begin{aligned}
& u_{x}(x, y, z)=-\sigma x \frac{\partial \phi}{\partial z}(z) \\
& u_{y}(x, y, z)=-\sigma y \frac{\partial \phi}{\partial z}(z) \\
& u_{z}(x, y, z)=\phi(z)
\end{aligned}
$$

torsion:

$$
\begin{aligned}
& u_{x}(x, y, z)=-y \phi(z) \\
& u_{y}(x, y, z)=x \phi(z) \\
& u_{z}(x, y, z)=\psi(x, y) \frac{\partial \phi}{\partial z}(z)
\end{aligned}
$$

\section{vertical bending:}

$$
\begin{aligned}
& u_{x}(x, y, z)=\phi(z)+\frac{\sigma}{2}\left(x^{2}-y^{2}\right) \frac{\partial^{2} \phi}{\partial z^{2}}(z) \\
& u_{y}(x, y, z)=\sigma x y \frac{\partial^{2} \phi}{\partial z^{2}}(z) \\
& u_{z}(x, y, z)=-x \frac{\partial \phi}{\partial z}(z)+\sigma \chi_{v}(x, y) \frac{\partial^{3} \phi}{\partial z^{3}}(z) .
\end{aligned}
$$

In the above, the coordinate system is oriented along the principal axes of inertia, $(x, y)=0$ corresponds to the center of mass of the cross section, and $\sigma$ is the Poisson ratio of the beam's material. The functions $\psi(x, y)$ and $\chi_{v}(x, y)$ are determined by the static equation $\nabla \cdot \boldsymbol{\sigma}=0$, the free boundary conditions, and the condition $\left\langle u_{z}\right\rangle_{S}=$ 0 . In the case of bending these yield

$$
\begin{aligned}
\frac{\partial \chi_{v}}{\partial x}(x, y) & =-\frac{\partial \tilde{\chi}_{v}}{\partial y}(x, y)+\frac{x^{2}+y^{2}}{2 \sigma} \\
\frac{\partial \chi_{v}}{\partial y}(x, y) & =\frac{\partial \tilde{\chi}_{v}}{\partial x}(x, y)+\frac{x y}{\sigma} \\
\left\langle\chi_{v}(x, y)\right\rangle_{S} & =0
\end{aligned}
$$

where $\tilde{\chi}_{v}$ is an harmonic function that at the cross section's boundary $(x[l], y[l])$ satisfies

$$
\begin{aligned}
\tilde{\chi}_{v}(l)= & \frac{1}{6 \sigma}\left[3(1+\sigma) x^{2} y+(1-\sigma) y^{3}\right]_{0}^{l} \\
& -\frac{2(1+\sigma)}{\sigma} \int_{0}^{l} x y \frac{\mathrm{d} x}{\mathrm{~d} l^{\prime}} \mathrm{d} l^{\prime} .
\end{aligned}
$$

The analogous relations for the case of torsion are given in Reference [45] while the transverse profile of the displacement field for horizontal bending can be obtained from the RHS of Eq. (B4) and Eqs. (B5), (B6) via the ansatz $x \leftrightarrow y, \chi_{v}(y, x) \rightarrow \chi_{h}(x, y), \tilde{\chi}_{v}(y, x) \rightarrow \tilde{\chi}_{h}(x, y)$.

Finally, the desired approximation for $\bar{A}_{\beta, 0}[x, y, d,-i k]$ is obtained from Eqs. B2 $-(\mathrm{B} 4)$ via the replacement

$$
\frac{\partial^{n} \phi}{\partial z^{n}} \rightarrow \begin{cases}\frac{1}{\sqrt{2 \pi S}}(i k)^{n} & \text { for } \beta=c, v, h \\ \frac{1}{\sqrt{2 \pi I_{z}}}(i k)^{n} & \text { for } \beta=t\end{cases}
$$

where we have taken into account that the 1D continuum modes $\left|v_{\beta}^{(0)}(k)\right\rangle$ are normalized in the standard Euclidean metric. Whence, we define

$$
\phi_{k(q), \beta}(z)= \begin{cases}\frac{\sqrt{2 \pi S}}{t_{q}} u_{z, q}(0,0, z) & \text { for } \beta=c \\ \frac{\sqrt{\pi I_{z}}}{\sqrt{2} t_{q}} \hat{z} \cdot \nabla \times \bar{u}_{q}(0,0, z) & \text { for } \beta=t \\ \frac{\sqrt{2 \pi S}}{t_{q}} u_{x / y, q}(0,0, z) & \text { for } \beta=v / h .\end{cases}
$$

The corresponding approximate dispersion relations (4) are specified by

$$
\tilde{c}_{c}=\sqrt{\frac{E_{b}}{\rho_{b}}}, \quad \tilde{c}_{t}=\sqrt{\frac{C}{\rho_{b} I_{z}}}, \quad \tilde{c}_{v / h}=\sqrt{\frac{E_{b} I_{y / x}}{\rho_{b} S}} .
$$

Here $I_{i}$ are the moments of inertia with respect to the principal axes of a cross section per unit surface density, $C$ is the torsional rigidity of the beam and $E_{b}$ its Young's modulus.

\section{End corrections}

If one considers now a semi-infinite beam $(z>0)$ it is clear that complex values for $k$ (the wavevector along $z$ ) are physically meaningful provided that their imaginary part is positive. The corresponding solutions can be understood as the analytic continuation of the "traveling wave" eigenmodes of an indefinite beam. More precisely, $\omega^{2}(k, d)$ and the transverse profile $\bar{A}(x, y, k, d)$ will be multivalued analytic functions of $k$ that yield harmonic solutions on the paths in the complex plane specified by $\Re\left[\omega^{2}(k, d)\right] \geq 0, \Im\left[\omega^{2}(k, d)\right]=0$. This can be viewed explicitly for a cylindrical cross section given that the resulting problem is separable (see Ref. [46] and references therein) but will hold in general provided that the boundary of the cross section is well behaved. We assume for simplicity that the latter is characterized by a single length scale $d$ but all our considerations follow for an arbitrary cross section (within the caveats that follow) provided that we reinterpret $d$ as the largest chord. Heuristic considerations imply that for a generic geometry the finite complex zeroes of $\omega^{2}(k, d)$ will have a real part that is at least of order $1 / d$. We will further assume that at these zeroes $\omega^{2}(k, d)$ is non-singular (i.e. they are not branch points) and has non-zero derivative; and that the associated path yielding physical solutions admits a parametrization in terms of $\Re[k]$ or $\Im[k]$ that can be Taylor expanded. Thus each of these non-trivial zeros yields a branch of end corrections (with labels $\beta, m$ as defined in Subsec. IV A specified by the transverse profiles $\bar{A}_{\beta, m}\left[x, y, d, \kappa_{\beta, m}\left(\omega^{2}, d\right)\right]$ and the "wavevectors along $z$ " $\kappa_{\beta, m}\left(\omega^{2}, d\right) \equiv-i k$, where the functions $\bar{A}_{\beta, m}, \kappa_{\beta, m}$ can be expanded in natural powers of $\omega^{2}$.

To analyze the problem of transmission at a $3 \mathrm{D}-1 \mathrm{D}$ junction it proves useful to consider the overall displacement and rotation of a given cross section. The former is 
simply defined as the displacement of the cross section's center of mass. In turn, a natural definition of a spatially averaged angle for a neighborhood $V$ undergoing harmonic motion is afforded by considering the maximum total angular momentum $\bar{L}_{V}$ with respect to the center of mass $\bar{r}_{V}$ of $V$ over a period:

$$
\langle\bar{u}(\bar{r})\rangle_{\mathrm{ang}, V} \equiv \frac{1}{\omega} \mathbb{I}^{-1} \bar{L}_{V}
$$

where $\mathbb{I}$ is the equilibrium inertia tensor for $V$. If we orient the axes along the principal axes of inertia and set the origin at $\bar{r}_{V}$, we obtain:

$$
\langle\bar{u}(\bar{r})\rangle_{\mathrm{ang}, V} \cdot \hat{i}=\frac{\int_{V} \mathrm{~d} r^{3} \bar{r} \times \bar{u}(\bar{r}) \cdot \hat{i}}{\int_{V} \mathrm{~d} r^{3}\left(r^{2}-x_{i}^{2}\right)} .
$$

If $V$ is small enough this reduces to

$$
\langle\bar{u}(\bar{r})\rangle_{\mathrm{ang}, V} \cdot \hat{i} \approx \frac{1}{2} \nabla \times \bar{u}(0) \cdot \hat{i}+\gamma_{i} u_{j k}(0) \text { with } \epsilon_{i k j}=1
$$

where $\gamma_{i} \equiv \sum_{j, k} \epsilon_{i j k}\left(I_{k}-I_{j}\right) / 2 I_{i}$ and the relative order of the corrections is at most $u(0) d / \min \left\{\left|u_{i j}(0)\right|\right\}$ with $d$ the typical dimension of the neighborhood $V$. We note that throughout Sec. IV we use the notation

$$
\bar{\theta} \equiv\langle\bar{u}(\bar{r})\rangle_{\mathrm{ang}, S}
$$

where $S$ is the beam's cross section at the origin.

In the limit $\omega \rightarrow 0$ each branch of end corrections yields an exponentially decaying solution $\left(\propto e^{-\kappa_{\beta, m}(0, d) z}\right)$ of the corresponding static problem (i.e. a static end correction). To establish Eqs. (46) underpinning our analysis of a 3D-1D junction, in addition to the aforementioned analyticity properties, we just need to use the following exact universal properties of static end corrections:

$$
\left\langle\mathbf{F} \cdot\left\{\bar{A}_{\beta, m}\left[x, y, d, \kappa_{\beta, m}(0, d)\right] e^{-\kappa_{\beta, m}(0, d) z}\right\}\right\rangle_{S}=0
$$

$$
\left\langle\mathbf{F} \cdot\left\{\bar{A}_{\beta, m}\left[x, y, d, \kappa_{\beta, m}(0, d)\right] e^{-\kappa_{\beta, m}(0, d) z}\right\}\right\rangle_{\text {ang }, S}=0
$$

$$
\left\langle\bar{A}_{\beta, m}\left[x, y, d, \kappa_{\beta, m}(0, d)\right]\right\rangle_{S}=0
$$

$$
\left\langle\bar{A}_{\beta, m}\left[x, y, d, \kappa_{\beta, m}(0, d)\right]\right\rangle_{\text {ang }, S}=0
$$

$$
\begin{array}{r}
\left\langle\int _ { S } \mathrm { d } { r ^ { \prime } } ^ { 2 } \mathbf { G } ( \overline { r } - \overline { r } ^ { \prime } , 0 ) \cdot \mathbf { F } \cdot \left\{\bar{A}_{\beta, m}\left[x^{\prime}, y^{\prime}, d, \kappa_{\beta, m}(0, d)\right]\right.\right. \\
\left.\left.\times e^{-\kappa_{\beta, m}(0, d) z^{\prime}}\right\}\right\rangle_{S}=0
\end{array}
$$

$$
\begin{aligned}
\left\langle\int_{S} \mathrm{~d}{r^{\prime}}^{2} \mathbf{G}\left(\bar{r}-\bar{r}^{\prime}, 0\right) \cdot \mathbf{F} \cdot\right. & \left\{\bar{A}_{\beta, m}\left[x^{\prime}, y^{\prime}, d, \kappa_{\beta, m}(0, d)\right]\right. \\
& \left.\left.\times e^{-\kappa_{\beta, m}(0, d) z^{\prime}}\right\}\right\rangle_{\text {ang }, S}=0,
\end{aligned}
$$

where now $\mathbf{G}(\bar{r}, 0)$ is the static Green's function of a free elastic half-space loaded at the free surface. Equations (B14) and (B15) correspond, respectively, to the total force and torque applied at $S$ and can be established by simply stating the equilibrium conditions for a given finite segment of the beam. In fact the exponential dependence on $z$ implies that the total external force and total external torque applied to the finite segment result, respectively, proportional to the L.H.S of Eqs. (B14) and B15.

On the other hand Equations $(\mathrm{B} 16)$ and $(\mathrm{B} 17)$ can be reduced to the following Lemma:

Lemma: For a finite beam of length $L$ in equilibrium subject to specified displacements at $z=0$ and free boundary conditions at $z=L$ the average displacements and angles at both ends coincide,

by taking the displacements at $z=0$ specified by $\bar{A}_{\beta, m}\left[x, y, d, \kappa_{\beta, m}(0, d)\right]$ and then sending $L \rightarrow \infty$. In turn, the above Lemma can be understood using a variational argument given that the elastic energy density is positive definite in the relative distances between the material points of the beam (see Ref. 46 and references therein). Equations (B14) through (B17) underly the standard recipes for the boundary conditions in the TRE treatment of static small deflections (i.e. "linearizedstrain" theory 45 . In this respect, it is important to note that for a beam in equilibrium the corresponding TRE solutions, already discussed, are exact within the linearized-strain 3D theory. Thus the validity of the latter recipes hinges on whether the strain can be linearized and not on the smallness of $d / L$.

We now consider the cantilever geometry (cf. Fig. 1) for an arbitrary length $L$ and apply the stress source associated to a given static end correction $(\beta, m)$ at $z=L$, the usual free boundary conditions at the other surfaces and fixed displacement boundary conditions for $z \rightarrow-\infty$ in the support (the latter ensues for $\omega \rightarrow 0$ given the boundary conditions satisfied by the support's Green's function). Equations (B14)-(B15) imply that the TRE part of the corresponding static solution inside the beam [cf. Eq. (B2)-(B4)] is at most an overall displacement and rotation. However a variational argument analogous to the one underlying the above Lemma implies that this displacement and rotation of the beam should vanish so that the solution is given solely by "end corrections". Then, continuity at the junction yields Eqs. B18- B19 if we take the limit $L \rightarrow 0$. We note that this derivation will be valid for any well behaved support irrespective of any symmetries.

\section{APPENDIX C: GENERALIZATIONS TO ASYMMETRIC STRUCTURES, 2D SLAB SUPPORTS, AND SWNTS}

The derivations in Subsec. IV A rely on the assumption that both the support and the beam are symmetric under reflections with respect to the $x$ and $y$ axes. It is straight- 
forward to extend these results to the case where only the beam's cross section presents these symmetries so long as we assume that the (now asymmetric) support is still characterized by a structureless continuum of free eigenmodes $\bar{u}_{q}^{(0)}(\bar{r})$ that has an effective 3D density of states 82 . More specifically each mode $\bar{u}_{q}^{(0)}(\bar{r})$ should be characterized by a single length scale of order $1 / q_{t}$ (where $q_{t}\left(\omega_{q}\right)$ is the wavevector for propagation of transverse elastic waves in the support's material). Thus the analogous property will hold for the corresponding Green's function $\mathbf{G}\left(\bar{r}, \bar{r}^{\prime}, \omega\right)\left(\bar{r}^{\prime}\right.$ lies at the boundary where the normal stress vanishes when the support is unloaded). A simple example would be an elastic quarter-space or any fraction of an elastic space with origin at the junction subtending a solid angle of order $\pi / 2$. In this more general scenario there will be mode mixing between the branches due to the scattering at the support. Equation (38) will still be valid with the analogous definition for $\bar{u}_{*}$ but now in Eq. (39) the decompositions of the fields $\Delta \bar{u}_{+}$and $\bar{u}_{*}$ in terms of the eigenmodes of the beam will involve an additional sum over all the branches $\beta$ (i.e. $\sum_{m} \rightarrow \sum_{m, \beta}$ ). Concomitantly these fields will no longer have a branch index except for $\bar{u}_{*}$ in case $(i)$ where $\beta^{\prime}$ will denote the character of the associated incident field $\bar{u}_{\mathrm{in}, \beta^{\prime}}$, and the analog of Eq. 45 will no longer involve a symmetrization operator $S_{\beta}$. The support mode labels $q$ no longer bear any relation with the beam branch index $\beta$ and now a convenient parametrization of the complete solution is afforded by $q_{t}$ instead of $k$.

In complete analogy to the symmetric case we substitute the modified decompositions (39) into the modified Eq. (45) and Eq. (38) and take on both sides the spatial averages $\langle\ldots\rangle_{S}$ and $\langle\ldots\rangle_{\text {ang }, S}$. Note that the beam's symmetries imply that on the LHS of each of the resulting equations for the amplitudes $c_{\beta m}^{(*)}$ and $c_{\beta m}$ only the term with the relevant $\beta$ - i.e. the one associated with the TRE branch for which the corresponding average does not vanish - will survive. Once again we solve for the TRE starred amplitudes in the linear system arising from the analog of Eq. (45) and substitute them into the linear system arising from the analog of Eq. (38), whence we solve for the un-starred TRE amplitudes. As before the end corrections yield terms that scale at most as the inertia $\left(q_{t} d\right)^{2}$. Thus, we find that — with the noticeable exception of the un-starred torsional TRE amplitude for case $(i i)$ - the results for the amplitudes $c_{\beta m}^{(*)}$ and $c_{\beta m}$ are given by Eqs. (44) and (47)-(50) with the following straightforward modifications: $k d$ is replaced by $\sqrt{q_{t} d}$, $\bar{u}_{q}^{(0)}(\bar{r})$ denotes now the free modes of the "generic" support, and in case $(i)$ the RHS of the equations for the starred TRE amplitudes are now multiplied by $\delta_{\beta, \beta^{\prime}}$.

The caveat for incidence from the support [case $(i i)$ ] is that now $c_{t, 0}\left(q_{t} d\right)$ has a contribution comparable to $c_{t, 0}^{(*)}\left(q_{t} d\right)$ of order $q_{t} d$ arising from the other TRE branches. Thus in this case Eq. 41) is no longer valid. However the correction to the approximate transmission amplitude $t_{q, t}^{(0)}$ (whose scaling as $q_{t} d$ is preserved) arising from $c_{t, 0}\left(q_{t} d\right)$ can be readily added to yield

$$
\begin{aligned}
t_{q, t}^{(0)}= & \frac{\partial c_{t, 0}^{(*)}}{\partial q_{t}}(0) q_{t}+\sum_{\{\beta \neq t, m\} \in \mathrm{TRE}} \sqrt{2 \pi I_{z}} \hat{z} \\
& \cdot\left\langle\int_{S} \mathrm{~d}{r^{\prime}}^{2} \mathbf{G}\left(\bar{r}, \bar{r}^{\prime}, 0\right) \cdot \hat{z} f_{\beta, m}^{(0)}\left(\bar{r}^{\prime}\right)\right\rangle_{\mathrm{ang}, S} c_{\beta m}^{(*)}(0)
\end{aligned}
$$

with

$$
\begin{aligned}
f_{v / h, m}^{(0)}\left(x_{1}, x_{2}\right) & =(-1)^{m} \frac{E_{b}}{\sqrt{2 \pi S}} x_{1 / 2} k_{v / h}^{2}\left(q_{t}\right) \quad m=0,1 \\
f_{c, 0}^{(0)}\left(x_{1}, x_{2}\right) & =i \frac{E_{b}}{\sqrt{2 \pi S}} k_{c}\left(q_{t}\right)
\end{aligned}
$$

where we have used the transverse profiles of the TRE modes given in App. $\mathrm{B}$ and $\mathbf{G}\left(\bar{r}, \bar{r}^{\prime}, 0\right)$ is now the static Green's function for the asymmetric support under consideration.

The aforementioned results for the starred amplitudes imply that for incidence from the beam to lowest order in $q_{t} d$ there is no mixing between the branches so that the reflection amplitudes for $\beta \neq \beta^{\prime}$ vanish and those for $\beta=\beta^{\prime}$ coincide with the $r_{c / t}^{(L)}\left[k\left(q_{t}\right) d\right], r_{v / h, \delta \eta}^{(L)}\left[k\left(q_{t}\right) d\right]$ already found for the symmetric case. On the other hand for incidence from the support the solution is a superposition of all four branches with approximate amplitudes $t_{q, \beta}^{(0)}$ given by Eq. $\mathrm{C} 1$ and for $\beta \neq t$ the same results as in Subsec. IV A in terms of the modified $\bar{u}_{q}^{(0)}(\bar{r})$. If we now revise the treatment of the modes of the whole structure given in Sec. III the fact that the branches do not mix implies that to lowest order the displacement field inside the beam (and away from the junctions) is just given by adding coherently the four contributions of the TRE branches which are given by similar expressions as in the symmetric case but with modified transmission amplitudes $t_{q, \beta}^{(0)}$ (i.e. the reflection amplitudes and the ratio $b$ remain the same). Furthermore, the symmetries of the beam imply that a resonator mode of branch $\beta$ only has non-vanishing overlap with the contribution to a scattering mode of the same branch so that the quantities $\left\langle u_{R}^{\prime} \mid u_{q}\right\rangle$ (to lowest order in $q_{t} d$ ) coincide with those for the symmetric supports except for the modification in the prefactor $t_{q, \beta}^{(0)}$. Thus, in general the results for the force spectral densities (cf. Sec. VB) that we will extract from these overlaps will be completely analogous to those for the fully symmetric case.

The only caveat is when there are degeneracies between resonances of different branches, i.e. $\omega_{m, \beta}=\omega_{n, \beta^{\prime}}=\omega_{R}$ with $\beta \neq \beta^{\prime}$. Unlike the fully symmetric case there is now mode mixing which, albeit higher order in $d / L$, may nonetheless invalidate our approximations for the functions $I_{m, \beta}(\omega), I_{n, \beta^{\prime}}(\omega)$ in a neighborhood of $\omega_{R}$ (cf. Sec. VB and App. F). The situation is completely equivalent to the failure of our treatment for $I_{n, \beta}\left(\omega_{m, \beta}\right)$ 
with $n \neq m$ with the difference that now the syndrome occurs precisely where the function is more relevant. On the other hand it is simple to realize that this breakdown is completely consistent with the nature of our approximation which is perturbative in $d / L$. If the asymmetry removes the selection rules that prevented the supports from coupling the two degenerate modes, the CaldeiraLeggett Hamiltonian provided by Eq. 20 with a single discrete resonator mode no longer affords a convenient representation as $d / L \rightarrow 0$. In particular, this will affect the two bending branches for cross sections symmetric under rotations by $\pi / 2$ whenever the supports break the $\hat{R}_{y}$ symmetry [cf. Eq. 14 and Fig. 1]. Thus, in a realistic scenario (i.e. at finite $d / L$ and finite mode-splitting) our results for the bending resonances $\omega_{n, v / h}$ will only be applicable to the case of SWNTs and nanowires (discussed below) provided the mode-splitting is negligible compared with the natural linewidth $\omega_{n, v / h} / Q_{n, v / h}$ induced by the phonon tunneling.

We have also analyzed the case when the cross section is also asymmetric following the analogous procedure. The key point in this further extension is that the lowest order contributions in $k d$ to the TRE mode profiles given in App. B satisfy the reflection symmetries for arbitrary cross section (with the axes oriented along the principal axes of inertia). Thus when the starred amplitudes are zeroth order in $q_{t} d$ the overlaps $\left\langle u_{R}^{\prime} \mid u_{q}\right\rangle$ will once again coincide [to lowest order in $q_{t} d$ and up to a modification in the prefactor $\left.t_{q, \beta}^{(0)}\right]$ with those for the symmetric supports. It follows that the extension to asymmetric cross sections carries over for the results that will ensue for the force spectral densities (cf. Sec. V B) except for torsional resonances - in which case the relevant transmission amplitudes are higher order — and the aforementioned case of degeneracies.

Furthermore, we have used the same framework to analyze the analogous problem of an abrupt junction between a rectangular beam of width $w$ and a slab (support) of the same thickness $33 \mathrm{t} \ll \mathrm{w} \equiv d$. Thus, we can use instead of $3 \mathrm{D}$ elasticity the $2 \mathrm{D}$ effective theory provided by "thin plate elasticity" $33 \mid 45$ adequate for phonon wavelengths much larger than t. A completely analogous treatment is feasible based on the 2D analog of Eqs. (38), 39, with: $(i) \bar{u}_{q}^{(0)}(y, z)$ and $\mathbf{G}(y, z, \omega)$ denoting now, respectively, the free modes and retarded Green's function of the two dimensional elastic half-plane (i.e. a semiinfinite thin plate lying on the $y z$-plane in our notation), and suitable redefinitions of (ii) the operator $\mathbf{F}$ and (iii) the beam's mode profiles $\bar{A}_{\beta, m}\left[y, d, \kappa_{\beta, m}(k, d)\right]$ which are now one-dimensional. The reflection symmetries also apply in this case so that the vertical bending and torsional branches of the beam only couple to the flexural modes of the plate while its horizontal bending and compressional branches only couple to the in-plane modes of the latter. Thus one can prove the same results for the reflection amplitudes and obtain results analogous to Eqs. (47), (50) for the transmission amplitudes $t_{q}, b t_{q}$ in terms of the averaged displacements and angles of the slab sup- port - the redefinitions (iii) imply that in the corresponding cross-section-dependent prefactors one has to replace $S \rightarrow \mathrm{w}$ and $I_{z} \rightarrow \mathrm{w}\left\langle r^{2}\right\rangle_{S}$ (cf. Subsec. IA). The only caveat is that for vertical bending the second relation for the starred amplitudes in Eq. (49) is no longer homogeneous since now the averaged angles $\theta_{y}$ scale as $\sqrt{\omega}$ for the relevant "free modes" of both the beam and the plate (flexural modes). This leads to a modification of the ratio $b$ for vertical bending that now may acquire a $q$-dependence. As a consequence of the above analogy the relations $(5][(6)]$ between the spectral densities $I_{n, \beta}(\omega)$ and the effective environmental DOS of the support $\tilde{\rho}_{\beta}(\omega)$ [transmission coefficients $\tau_{\beta}(\omega)$ ] will be the same (in terms of the analogous functions for the thin plate "support") as in the 3D case with the exception of the expression for $C_{n, v}$ that will be modified for small $n$ - for large $n$ the large decay length exponentials can be neglected and the dependence on the ratio $b$ of $I_{n, \beta}(\omega)$ becomes negligible.

Finally, we turn to the case of SWNTs and nanowires. One should note that as there is no valid underlying "microscopic" theory applicable to both the supports and the beam the precise analog of Eq. 38 will in general be unknown, depending on details of the clamping procedure. However, one can argue that insofar as there is no dissipation inside the "junction" the above results for the reflection and transmission amplitudes will still hold - with the straightforward replacements in Eqs. (47), (49), (50) that result from the redefinition of the transverse mode profiles: $S \rightarrow N_{c}, I_{z} \rightarrow N_{c}\left\langle r^{2}\right\rangle_{S}$, with $N_{c}$ the number of atoms in the unit cell 83 (of the appropriate equivalent $1 \mathrm{D}$ chain in the case of a nanowire $\left.{ }^{47}\right)$. In particular, one should note that the analog of the universal properties of the end corrections (cf. App. B are expected to hold if the SWNT or nanowire is to be regarded as "clamped".

\section{APPENDIX D: EXTENSION TO MICROTOROIDS AND OTHER GEOMETRIES}

The facts that the resonator mode $\left|u_{R}^{\prime}\right\rangle$ and the scattering modes $\left|u_{q}\right\rangle$ are solutions of the time-independent elastic wave equations (cf. Sec. II), and that $\bar{u}_{R}^{\prime}(\bar{r})$ is real, directly imply

$$
\begin{aligned}
\omega_{q}^{2}\left\langle u_{R}^{\prime} \mid u_{q}\right\rangle & =-\frac{1}{\rho_{s}} \int_{V_{R}} \mathrm{~d} r^{3} u_{R, i}^{\prime} \frac{\partial \sigma_{q, i j}}{\partial x_{j}} \\
\omega_{R}^{2}\left\langle u_{R}^{\prime} \mid u_{q}\right\rangle & =-\frac{1}{\rho_{s}} \int_{V_{R}} \mathrm{~d} r^{3} u_{q, i} \frac{\partial \sigma_{R, i j}^{\prime}}{\partial x_{j}}
\end{aligned}
$$

where we use Einstein's sum convention and that $\bar{u}_{R}^{\prime}(\bar{r})$ only has support in $V_{R}$. The above can be re-expressed as

$$
\omega_{q}^{2}\left\langle u_{R}^{\prime} \mid u_{q}\right\rangle=-\frac{1}{\rho_{s}} \int_{V_{R}} \mathrm{~d} r^{3}\left[\frac{\partial}{\partial x_{j}}\left(u_{R, i}^{\prime} \sigma_{q, i j}\right)-u_{R, i j}^{\prime} \sigma_{q, i j}\right]
$$




$$
\omega_{R}^{2}\left\langle u_{R}^{\prime} \mid u_{q}\right\rangle=-\frac{1}{\rho_{s}} \int_{V_{R}} \mathrm{~d} r^{3}\left[\frac{\partial}{\partial x_{j}}\left(u_{q, i} \sigma_{R, i j}^{\prime}\right)-u_{q, i j} \sigma_{R, i j}^{\prime}\right]
$$

by using that the stress tensor is symmetric and that the contraction of a symmetric tensor with an antisymmetric one vanishes. As the mapping of the strain $u_{i j}$ onto the stress $\sigma_{i j}$ corresponds to a symmetric matrix (given that its entries are the second derivatives of the elastic energy with respect to the strain components) we have

$$
u_{R, i j}^{\prime} \sigma_{q, i j}=u_{q, i j} \sigma_{R, i j}^{\prime} .
$$

Substracting Eq. (D4) from Eq. (D3) and using Eq. (D5), the divergence theorem, and the free boundary conditions satisfied by the displacement fields at the free surfaces of the resonator we obtain

$$
\left\langle u_{R}^{\prime} \mid u_{q}\right\rangle=\frac{\int_{S} \mathrm{~d} r^{2}\left(\bar{u}_{R}^{\prime} \cdot \boldsymbol{\sigma}_{q}-\bar{u}_{q} \cdot \boldsymbol{\sigma}_{R}^{\prime}\right) \cdot \hat{n}}{\rho_{s}\left[\omega_{R}^{2}-\omega^{2}(q)\right]}
$$

which together with Eqs. (26) and 82 finally yields Eq. 10, namely

$$
\begin{aligned}
I(\omega) \approx \frac{\pi}{2 \rho_{s}^{2} \omega_{R} \omega} \int_{q} \mid & \left.\int_{S} \mathrm{~d} r^{2}\left(\bar{u}_{R}^{\prime} \cdot \boldsymbol{\sigma}_{q}-\bar{u}_{q} \cdot \boldsymbol{\sigma}_{R}^{\prime}\right) \cdot \hat{n}\right|^{2} \\
& \times \delta[\omega-\omega(q)] .
\end{aligned}
$$

The above is very general as it only assumes $\left|\Delta_{I}\left(\omega_{R}\right)\right| / \omega_{R} \ll 1$ to ensure the validity of Eq. 82 .

We now focus on an axially symmetric structure consisting of a "resonator volume" supported by a vertical pedestal. Concrete relevant realizations of this geometry are microtoroids (cf. Fig. 2), microdisks and microspheres. We consider the regime in which the contact area $S$ between the resonator volume and the pedestal satisfies $S \ll h D$ and $\sqrt{S} \lesssim h$, where $D$ and $h$ are, respectively, the largest diameter and smallest characteristic dimension of the former. Thus there will be axially symmetric resonances with typical wavevector ${ }^{84} k_{R}$ that satisfy $\sqrt{S} k_{R} \ll 1$. We focus on one of them ${ }^{[55}$ and assume that it is "isolated" (cf. Subsec. IA). The limit $S \rightarrow 0$ yields a finite resonator volume subject to free boundary conditions on its whole surface, which specifies the natural choice of boundary conditions for the corresponding resonator mode ${ }^{86} \bar{u}_{R}^{\prime}$. This directly implies that the terms involving $\boldsymbol{\sigma}_{R}^{\prime}$ vanish in Eqs. (D6), 10) while property $\sqrt{S} k_{R} \ll 1$ allows us to factor $\bar{u}_{R}^{\prime}$ out of the integral which reduces then to the total force across $S$ associated with the scattering mode $q$.

We assume that for studying the propagation of modes with wavevector $q \sim k_{R}$ the pedestal can be modeled as an infinite beam with slowly varying cross section $q S(z) / S^{\prime}(z) \gg 1$. This should capture the adiabatic limit of perfect impedance-match with the substrate for the purpose of studying the dissipation. In turn, our prior treatment of a 3D-1D junction (cf. Sec. IV) can be extended to our present context (with the roles of support and resonator interchanged). Now the $3 \mathrm{D}$ object (resonator volume) presents finite dimensions comparable to the relevant wavevectors. Naturally, providing rigorous derivations (as the ones given in Sec. IVP) is now complicated by the lack of an explicit expression for the Green's function of the 3D object. Nonetheless, heuristic considerations imply that: (i) in the limit $\sqrt{S} q \ll 1$ with $\sqrt{S} \lesssim h$ the scattering mode $\bar{u}_{q}$ can be asymptotically approximated in the pedestal by $\bar{u}_{*, q}$ (which corresponds to an incoming wave satisfying clamped boundary conditions at $S$ ) provided $\omega(q)$ is not close to a resonance $\omega_{n}$ of the resonator volume, and (ii) once again $I(\omega)$ is smooth in a neighborhood of $\omega_{R}$. The latter implies (as for the beam geometry) that the restriction to modes $\bar{u}_{q}$ with $\omega(q) \neq \omega_{n}$ is immaterial as it only invalidates our result for $I(\omega)$ at $\omega=\omega_{n} \neq \omega_{R}$. In turn, it is straightforward to extend the formalism in App. B to a beam with adiabatically varying cross section. Thus the universal properties of the end corrections imply that to lowest order in $\sqrt{S} q$ only the TRE part of $\bar{u}_{q}$ contributes to the aforementioned total force. The axial symmetry of the resonance further implies that only compressional modes yield a finite contribution $\frac{87}{}$. Thus we obtain (cf. Fig. 2)

$$
\int_{S} \mathrm{~d} r^{2} \bar{u}_{R}^{\prime} \cdot \boldsymbol{\sigma}_{q} \cdot \hat{n} \approx-E_{s} \sqrt{\frac{S}{2 \pi}} \bar{u}_{R}^{\prime}(0) \cdot \hat{z} \frac{\partial \phi_{q}}{\partial z}(0)
$$

where the corresponding effective one dimensional field satisfies 46

$$
\frac{\partial^{2} \phi_{q}}{\partial z^{2}}+\frac{S^{\prime}}{S} \frac{\partial \phi_{q}}{\partial z}+\frac{\rho_{s}}{E_{s}} \omega^{2}(q) \phi_{q}=0
$$

and the approximation by $\bar{u}_{*, q}$ implies $\phi_{q}(0)=0$, $\phi_{q}^{(-)}(0)=1-$ here we define $\phi_{q}^{(-)}\left[\phi_{q}^{(+)}\right]$as the incoming (outgoing) components of the scattering mode ${ }^{88}$ $q$. We adopt for simplicity an exponential dependence $S(z)=S e^{-\Gamma z}$ which leads to the following

$$
\begin{aligned}
& \phi_{q}(z)=\phi_{q}^{(-)}(z)-\phi_{q}^{(+)}(z), \\
& \phi_{q}^{(\mp)}(z)=e^{\left(\frac{\Gamma}{2} \pm i q\right) z} \quad \text { with } \quad q=\sqrt{\frac{\rho_{s}}{E_{s}} \omega^{2}(q)-\frac{\Gamma^{2}}{4}}, \\
& \frac{\mathrm{d} q}{\mathrm{~d} \omega}=\frac{\rho_{s} \omega(q)}{E_{s} \sqrt{\frac{\rho_{s}}{E_{s}} \omega^{2}(q)-\frac{\Gamma^{2}}{4}}} .
\end{aligned}
$$

Then, substitution of Eqs. (D7), D9), D10 into Eq. 10 allows us to obtain

$$
I(\omega) \approx \sqrt{\frac{E_{s}}{\rho_{s}}} \frac{S}{\omega_{R}} u_{R, z}^{\prime 2} \sqrt{\omega^{2}-\omega_{I}^{2}} \Theta\left(\omega-\omega_{I}\right) .
$$

Clearly the infrared cutoff $\omega_{I}=\sqrt{E_{s} / \rho_{s}} \Gamma / 2$ is an artifact of the adiabatic assumption $q S(z) / S^{\prime}(z) \gg 1$ which implies $\omega \gg \omega_{I}$. In the latter appropriate limit we finally obtain Eq. 111 for the spectral density where $\tilde{u}_{R, z}(0)=\sqrt{m_{R} / \rho_{s}} u_{R, z}^{\prime}(0)$ corresponds to the resonator 
mode normalized to the "relevant coordinate" specific context $\Delta D / 2$ ). Note that, as expected, the result is independent of $\Gamma$. As for the beam geometry, for typical materials the $Q$ will be mostly a size-independent geometric property.

\section{APPENDIX E: DERIVATION OF $\Im\left\{\tilde{G}_{x x}(0)\right\}$ FOR THE ELASTIC HALF-SPACE}

In the following we define $\bar{u} \equiv \tilde{\mathbf{G}} \cdot \hat{x}$ and use dimensionless variables setting $q_{t} \equiv 1$. We need to solve for the half space $(z<0)$ :

$$
\nabla^{2} \bar{u}+\frac{1}{1-2 \sigma_{s}} \nabla(\nabla \cdot \bar{u})+\bar{u}=0
$$

subject to the following boundary conditions at $z=0$ :

$$
\begin{aligned}
& 2 u_{x z}=\delta(x) \delta(y) \\
& 2 u_{y z}=\frac{2}{1-2 \sigma_{s}}\left[\left(1-\sigma_{s}\right) u_{z z}+\sigma_{s}\left(u_{x x}+u_{y y}\right)\right]=0 .
\end{aligned}
$$

One can can construct the solution as a superposition of longitudinal $(j=1)$, $\mathrm{SH}(j=2)$ and $\mathrm{SV}(j=3)$ waves:

$$
\begin{aligned}
\bar{u}(x, y, z)= & \int_{-\infty}^{+\infty} \int_{-\infty}^{+\infty} \frac{\mathrm{d} q_{x} \mathrm{~d} q_{y}}{4 \pi^{2}} \bar{\varepsilon}_{j}\left(q_{x}, q_{y}\right) \tilde{u}_{j}\left(q_{x}, q_{y}\right) \\
& \times e^{i\left(q_{x} x+q_{y} y-\sqrt{\alpha_{j}-q_{x}^{2}-q_{y}^{2}} z\right)},
\end{aligned}
$$

where we have defined

$$
\alpha_{1}=\frac{c_{t}^{2}}{c_{l}^{2}}=\frac{1-2 \sigma_{s}}{2\left(1-\sigma_{s}\right)}=\alpha\left(\sigma_{s}\right), \quad \alpha_{2}=\alpha_{3}=1
$$

$$
\begin{array}{ll}
\bar{\varepsilon}_{1}=\left(q_{x}, q_{y}, q_{z, 1}\right), & \bar{\varepsilon}_{2}=\left(q_{y},-q_{x}, 0\right), \\
\bar{\varepsilon}_{3}=\left(-q_{z, 3} q_{x},-q_{z, 3} q_{y}, q_{x}^{2}+q_{y}^{2}\right) . &
\end{array}
$$

We note that $q_{z, j} \equiv-\sqrt{\alpha_{j}-q_{x}^{2}-q_{y}^{2}}$ can be imaginary, we do not need to normalize the $\bar{\varepsilon}_{j}$ for our purposes and $\alpha<1$.

In order to obtain the outgoing solution one can introduce damping, then calculate (E4) that will correspond to the steady state solution, and, finally, take the limit of damping coefficient going to zero. If we decompose the total displacement field into transverse and longitudinal components $\bar{u}=\bar{u}_{\perp}+\bar{u}_{\|}$, the modified equations of 3D elasticity can be written as

$$
\begin{gathered}
\frac{\partial^{2} \bar{u}_{\eta}}{\partial t^{2}}+\epsilon \frac{\partial \bar{u}_{\eta}}{\partial t}-c_{\eta}^{2} \nabla^{2} \bar{u}_{\eta}=0, \quad \eta=\perp, \| \\
\nabla \times \bar{u}_{\|}=0, \quad \nabla \cdot \bar{u}_{\perp}=0,
\end{gathered}
$$

with $\epsilon>0$, and $c_{\perp}=q_{t} c_{t}, c_{\|}=q_{t} c_{l}$. We look for solutions $\bar{u}(\bar{r}, t)=\bar{\varepsilon}_{j} e^{i\left(\bar{q}_{j} \cdot \bar{r}-\omega t\right)}$, which leads to:

$$
q_{j}^{2}=\frac{\omega^{2}}{c_{j}^{2}}\left(1+i \frac{\epsilon}{\omega}\right)
$$

with $c_{1}=c_{\|}$and $c_{2,3}=c_{\perp}$. These solutions can be obtained from the undamped ones by the replacement $\alpha_{j} \rightarrow \alpha_{j}(1+i \epsilon)$. The analysis of the limiting procedure $\epsilon \rightarrow 0$ allows the determination of the adequate integration contour $\mathcal{C}$ in the complex plane in the standard way.

If we substitute expression (E4) and Fourier transform we arrive at:

$$
\left[\begin{array}{ccc}
2 q_{x} q_{z, 1} & q_{y} q_{z, 2} & q_{x}\left(q_{x}^{2}+q_{y}^{2}-q_{z, 2}^{2}\right) \\
2 q_{y} q_{z, 1} & -q_{x} q_{z, 2} & q_{y}\left(q_{x}^{2}+q_{y}^{2}-q_{z, 2}^{2}\right) \\
\left(1-\sigma_{s}\right) q_{z, 1}^{2}+\sigma_{s}\left(q_{x}^{2}+q_{y}^{2}\right) & 0 & \left(1-2 \sigma_{s}\right) q_{z, 2}\left(q_{x}^{2}+q_{y}^{2}\right)
\end{array}\right]\left(\begin{array}{c}
\tilde{u}_{1} \\
\tilde{u}_{2} \\
\tilde{u}_{3}
\end{array}\right)=\left(\begin{array}{c}
-i \\
0 \\
0
\end{array}\right)
$$

We adopt polar variables $q_{x}=\rho \cos \varphi, q_{y}=\rho \sin \varphi$ and solve for the $\tilde{u}_{j}$

$$
\begin{array}{ll}
\tilde{u}_{1}(\rho, \varphi)=i\left(1-2 \sigma_{s}\right) \frac{\rho \sqrt{1-\rho^{2}+i \epsilon} \cos \varphi}{D(\rho)} & \tilde{u}_{2}(\rho, \varphi)=i \frac{\sin \varphi}{\rho \sqrt{1-\rho^{2}+i \epsilon}} \\
\tilde{u}_{3}(\rho, \varphi) & =i \frac{\left[\left(1-\sigma_{s}\right) \alpha\left(\sigma_{s}\right)+\left(2 \sigma_{s}-1\right) \rho^{2}\right] \cos \varphi}{\rho D(\rho)}
\end{array} \quad
$$

where

$$
D(\rho)=2\left(1-2 \sigma_{s}\right)\left[\rho^{2} \sqrt{1-\rho^{2}+i \epsilon} \sqrt{\alpha\left(\sigma_{s}\right)(1+i \epsilon)-\rho^{2}}+\left(\frac{1}{2}-\rho^{2}\right)\left(\frac{1+i \epsilon}{2}-\rho^{2}\right)\right] .
$$

If we consider the integral of expression (E4) evaluated at $z=0$ over the contour $\mathcal{C}$ we can identify a regular and a singular contribution to the displacement at the free boundary. We are only interested in the imaginary part of the 
$x$ component to which only the regular part contributes. Finally, evaluation at the origin $x=y=0$ leads to:

$$
\Im\left\{\tilde{G}_{x x}(0, \alpha)\right\}=\frac{1}{\pi} \mathscr{P}\left\{\int_{0}^{1} \mathrm{~d} v \frac{v^{2}\left(v^{2}-1 / 2\right)^{2}}{p\left(1-v^{2}\right)}-\int_{0}^{\sqrt{\alpha}} \mathrm{d} v v^{2} \frac{\left(\alpha-v^{2}\right)\left(v^{2}+1-\alpha\right)}{p\left(\alpha-v^{2}\right)}\right\}+R[\xi(\alpha), \alpha]+\frac{1}{4 \pi},
$$

where we have eliminated $\sigma_{s}$ in favor of $\alpha$, the contribution of the Rayleigh pole associated with the surface waves (SAW) is given by

$$
R[\xi, \alpha]=-\frac{1}{16 \xi} \frac{\left(1-\xi^{2} / 2\right)^{2} \sqrt{1-\xi^{2}}+\left(1-\xi^{2}\right) \sqrt{1-\alpha \xi^{2}}}{6(\alpha-1)+2(3-2 \alpha) \xi^{2}-\xi^{4}}
$$

and we have defined

$$
p(v) \equiv 16(\alpha-1) v^{3}+8(3-2 \alpha) v^{2}-8 v+1 .
$$

We note that the parameter $\xi$, already introduced in App. A, satisfies $\xi=1 / \sqrt{v_{*}}$, where $v_{*}$ is the only real root of E16 greater than unity.

\section{APPENDIX F: DERIVATION OF EQUATION (82)}

We will first invert Eq. (32) to obtain Eq. 81). To this effect we define a complex function $G(\omega)$ on the real axis such that for $\omega>0$

$$
\Im[G(\omega)]=-S(\omega) \text { and } \Re[G(\omega)]=\Delta_{S}(\omega)
$$

and the extension to $\omega<0$ is specified by

$$
G(-\omega)=G^{*}(\omega)
$$

which, after rearranging the integral for $\Delta_{S}(\omega)$ using the partial fraction expansion of $2 \omega^{\prime} /\left(\omega^{2}-\omega^{\prime 2}\right)$, yields for the whole real axis

$$
G(\omega)=\frac{1}{\pi} \mathscr{P} \int_{-\infty}^{\infty} \mathrm{d} \omega^{\prime} \frac{\operatorname{sgn}\left(\omega^{\prime}\right) S\left(\left|\omega^{\prime}\right|\right)}{\omega-\omega^{\prime}}-i \operatorname{sgn}(\omega) S(|\omega|)
$$

Given that $S(\omega)$ is well behaved $\left(C_{\infty}\right.$ for $\left.\omega>0\right)$ and given its behavior for $\omega \rightarrow 0$ and $\omega \rightarrow \infty$ discussed at the end of Subsec. VB, Eq. (F3) implies that the real and imaginary parts of $G(\omega)$ are Hilbert transforms of each other. Then, it follows from Titchmarsh's theorem $[61$ that $G(z)$ is analytic in the complex upper half-plane and that the integral over a semicircular contour in the latter tends to zero as the radius is increased. Thus we arrive at

$$
G(z)=\frac{1}{2 \pi i} \int_{-\infty}^{\infty} \mathrm{d} \omega \frac{G(\omega)}{\omega-z}
$$

valid for $\Im(z)>0$. We note that $G(\omega)$ gives the propagator of the resonator mode's canonical coordinate at zero temperature so that in physical terms the above properties of $G(z)$ can be understood as a consequence of causality - the inverse Fourier transform of $G(\omega)$ only has support for $t>0$. If we now substitute Eq. (F3) into Eq. (F4), interchange the order of the integrations over $\omega$ and $\omega^{\prime}$, perform the former, and rearrange the latter we obtain

$$
G(z)=\frac{1}{\pi} \int_{0}^{\infty} \mathrm{d} \omega^{\prime} \frac{2 \omega^{\prime}}{z^{2}-\omega^{\prime 2}} S\left(\omega^{\prime}\right)
$$

which with the help of Eq. 27) can be re-expressed as

$$
G(z)=\frac{1}{z^{2}}+\frac{\omega_{R}^{2}}{z^{4}}+\frac{1}{\pi z^{4}} \int_{0}^{\infty} \mathrm{d} \omega \frac{2 \omega^{5}}{z^{2}-\omega^{2}} S(\omega) .
$$

To proceed we define the function

$$
\Sigma(z) \equiv z^{2}-\omega_{R}^{2}-\frac{1}{G(z)},
$$

which using Eqs. F3, F1 and 32 can be shown to have the property

$$
\Im[\Sigma(\omega)]=\frac{-\operatorname{sgn}(\omega) S(|\omega|)}{\Delta_{S}^{2}(\omega)+S^{2}(|\omega|)}=-\omega_{R} \operatorname{sgn}(\omega) I(|\omega|) .
$$

As already discussed in Subsec. VB, $S(\omega)$ has a natural ultraviolet cutoff, given by $\omega_{*} \sim \omega_{\beta}(\pi / d)$, beyond which it is bounded by a power law $1 / \omega^{m}$ with $m \geq 6$. From this behavior one can derive that the last term in the RHS of Eq. $[\mathrm{F} 6]$ is bounded by $\ln \left|z / \omega_{*}\right| /|z|^{6}$ if $m=6$ and by $1 /|z|^{6}$ if $m>6$. This property together with Eqs. $\mathrm{F} 7$ and $(\mathrm{F} 6)$ directly implies that

$$
|\Sigma(z)| \lesssim \mathcal{O}\left[\frac{\ln \left|z / \omega_{*}\right|}{|z|^{2}}\right] \quad \text { or } \quad|\Sigma(z)| \lesssim \mathcal{O}\left[\frac{1}{|z|^{2}}\right]
$$

respectively, for $z \rightarrow \infty$. On the other hand Eq. $\mathrm{F} 5 \mathrm{im}$ plies that the propagator $G(z)$ has no zeros with $\Im(z)>0$ so that $\Sigma(z)$ is analytic in the upper half-plane. This property and Eq. (F9) allow us to obtain

$$
\Sigma(\omega)=\frac{1}{\pi i} \mathscr{P} \int_{-\infty}^{\infty} \mathrm{d} \omega^{\prime} \frac{\Sigma\left(\omega^{\prime}\right)}{\omega^{\prime}-\omega}
$$

using the Cauchy integral formula. If we now take the real part of this equation and use Eqs. (F7), (F3), (F1) and $\mathrm{F} 8 \mathrm{~s}$ we obtain after some simple rearrangements

$$
\Re[\Sigma(\omega)]=\omega_{R} \Delta_{I}(\omega)=\omega^{2}-\omega_{R}^{2}-\frac{\Delta_{S}(\omega)}{\Delta_{S}^{2}(\omega)+S^{2}(\omega)}
$$

for $\omega>0$. If we assume that the functions $\Delta_{I}(\omega)$ and $I(\omega)$ are known, Eq. (F11) together with Eq. (32) provide a system of algebraic equations for the unknowns $\Delta_{S}(\omega)$ 
and $S(\omega)$. Thus we can solve for $S(\omega)$ to finally obtain the desired result given by Eq. (81).

If we now consider Eqs. (F11) and (F8), we have

$$
\Sigma(\omega)=\omega_{R}\left[\Delta_{I}(\omega)-i \operatorname{sgn}(\omega) I(|\omega|)\right]
$$

that together with Eqs. 20 and 21) allow us to interpret the function $\Sigma(\omega) / 2 \omega_{R}$ as the resonator's self-energy induced by its interaction with the environment. Given the properties of $I(\omega)$ discussed below we may assume that $\Sigma(z)$ is analytic in a neighborhood of $z=\omega_{R}$. Thus, it follows from Eq. (F7) that any pole of $G(z)$ in that neighborhood is given by a root $z_{R}$ of the equation

$$
z^{2}-\omega_{R}^{2}-\Sigma\left(\omega_{R}\right)-\sum_{n=1}^{\infty} \frac{\partial^{n} \Sigma}{\partial \omega^{n}}\left(\omega_{R}\right)\left(z-\omega_{R}\right)^{n}=0 .
$$

In turn, the behavior of the function $S(\omega)$ discussed below [cf. Eq. (F25)] and Eq. (F3) imply that for $d / L \rightarrow 0$ the propagator $G(z)$ has indeed a pole in the lower halfplane close to $\omega_{R}$ that tends to $\omega_{R}$. If we now consider the asymptotic expansion of $\left(z_{R}-\omega_{R}\right) / \omega_{R}$ and $\Sigma(\omega) / \omega_{R}^{2}$ as a function of $d / L$ for $d / L \rightarrow 0$ and substitute it into Eq. (F13), it is straightforward to realize [cf. Eqs. (F8), (F11)] that $\left(z_{R}-\omega_{R}\right) / \omega_{R} \rightarrow 0$ directly implies the properties $\left|\Delta_{I}\left(\omega_{R}\right)\right| / \omega_{R} \rightarrow 0, I\left(\omega_{R}\right) / \omega_{R} \rightarrow 0$ used in Subsec. $\mathrm{VB}$ and in the derivation of Eq. 82 pursued below. Heuristically, this vanishing of the self-energy can be viewed as an unavoidable consequence of the behavior of the transmission coefficients $\tau(\omega)$ for $d / L \rightarrow 0$ unveiled in Sec. IV] In fact the interaction with the bath giving rise to the self-energy can be understood in terms of phonon tunneling between the beam and its supports, i.e. a mechanism that is suppressed as $\tau(\omega) \rightarrow 0$.

To proceed we reformulate Eq. 82 by defining $I_{*}(\omega)$ as its RHS. Thus, it is enough to prove

$$
I(\omega)=I_{*}(\omega)\left|1+\mathcal{O}\left[\frac{\left|\Delta_{I}\left(\omega_{R}\right)\right|}{\omega_{R}}\right]\right|^{2} .
$$

This can also be written as the condition $|E(\omega)|<$ $\left|\Delta_{I}\left(\omega_{R}\right)\right| / \omega_{R}$ where the function $E(\omega)$ is defined by

$$
E(\omega) \equiv \frac{\Delta_{I}(\omega)-\Delta_{I}\left(\tilde{\omega}_{R}\right)-i\left[I(\omega)-I\left(\tilde{\omega}_{R}\right)\right]-\frac{\omega-\tilde{\omega}_{R}}{\omega_{R}+\tilde{\omega}_{R}} \Delta_{I}\left(\tilde{\omega}_{R}\right)}{\omega-\tilde{\omega}_{R}+i \frac{\omega_{R}}{\omega_{R}+\omega} I\left(\tilde{\omega}_{R}\right)},
$$

[cf. Eq. 830] so that given Eq. 81 we have

$$
\frac{I(\omega)}{I_{*}(\omega)}=\left|1-\frac{\omega_{R}}{\omega_{R}+\omega} E(\omega)\right|^{2} .
$$

We note that Eq. 83 is just the real part of Eq. F13 restricted to the real axis. If we use again its asymptotic expansion in terms of the aspect ratio $d / L$ it is straightforward to derive from $\tilde{\omega}_{R} \rightarrow \omega_{R}$ when $d / L \rightarrow 0$ that $2\left(\tilde{\omega}_{R}-\omega_{R}\right) / \Delta_{I}\left(\omega_{R}\right) \rightarrow 1$, which together with Eq. (83) implies that $\Delta_{I}\left(\tilde{\omega}_{R}\right)$ and $\Delta_{I}\left(\omega_{R}\right)$ coincide to lowest order in the aspect ratio. It is worth noting that though the real part of the exact pole of $G(z)$ differs from $\tilde{\omega}_{R}$, as the imaginary part of Eq. $\mathrm{F} 13$ comes into play, analogous considerations yield $2\left(z_{R}-\omega_{R}\right) / \Delta_{I}\left(\omega_{R}\right) \rightarrow 1$ so that $\Delta_{I}\left(\omega_{R}\right) / 2$ provides the lowest order contribution to the renormalization of the bare frequency.

In order to ensure the validity of Eq. 82 we find that in addition to $\left|\Delta_{I}\left(\omega_{R}\right)\right| / \omega_{R} \ll 1$ the following assumptions (whose validity is discussed below) are needed:

$$
\left|\frac{\partial^{n} \Delta_{I}}{\partial \omega^{n}}(\omega)\right| \sim \frac{\left|\Delta_{I}(\omega)\right|}{\omega^{n}}, \quad\left|\frac{\partial^{n} I}{\partial \omega^{n}}(\omega)\right| \sim \frac{I(\omega)}{\omega^{n}},
$$

for $n=1,2$ and frequencies smaller than the ultraviolet cutoff $\omega_{*} \gg \omega_{R}$. Heuristic considerations imply that from Eq. (F17) and the behaviors of $I(\omega)$ for $\omega \rightarrow 0$ and $\omega \rightarrow \infty$ (discussed at the end of Subsec. VB), it follows that the only relevant frequency scale when considering the profile of $I(\omega)$ is $\omega_{*}$ near which this function attains its maximum. In turn this implies that

$$
\left|\Delta_{I}\left(\tilde{\omega}_{R}\right)\right| \approx\left|\Delta_{I}(0)\right| \sim I\left(\omega_{*}\right) \sim \max \{I(\omega)\},
$$

and that in the interval $\left(0, \omega_{*}\right), \frac{\partial I}{\partial \omega}(\omega)>0$. On the other hand we can use the mean value theorem to write

$$
\begin{aligned}
\pi \Delta_{I}(\omega)= & -\int_{\omega / 2}^{3 \omega / 2} \mathrm{~d} \omega^{\prime}\left[\frac{\partial I}{\partial \omega}\left(\Omega\left[\omega^{\prime}, \omega\right]\right)+\frac{1}{\omega+\omega^{\prime}} I\left(\omega^{\prime}\right)\right] \\
& +\left[\int_{0}^{\omega / 2}+\int_{3 \omega / 2}^{\infty}\right] \frac{\omega^{\prime}}{\omega-\omega^{\prime}} \frac{2}{\omega+\omega^{\prime}} I\left(\omega^{\prime}\right) \mathrm{d} \omega^{\prime}
\end{aligned}
$$

where $\Omega\left[\omega^{\prime}, \omega\right] \in\left(\omega, \omega^{\prime}\right)$. Equations $(\mathrm{F} 17)-(\mathrm{F} 19)$ imply

$$
\frac{\left|\Delta_{I}(\omega)\right|}{\omega}, \frac{I(\omega)}{\omega} \lesssim \frac{\left|\Delta_{I}\left(\tilde{\omega}_{R}\right)\right|}{\tilde{\omega}_{R}},
$$

for $\tilde{\omega}_{R} \leq \omega<\omega_{*}$, and

$$
\Delta_{I}(\omega) \approx \Delta_{I}\left(\tilde{\omega}_{R}\right), \quad I(\omega)<I\left(\tilde{\omega}_{R}\right) \lesssim\left|\Delta_{I}\left(\tilde{\omega}_{R}\right)\right|,
$$

for $\omega<\tilde{\omega}_{R}$.

Furnished with relations (F17), F20 and F21, we turn now to the analysis of $|E(\omega)|$ defined in Equation F15. First we consider the case $\left|\omega-\tilde{\omega}_{R}\right| \gtrsim \tilde{\omega}_{R}$. For $\omega>\tilde{\omega}_{R}$ we use $\left|\omega-\tilde{\omega}_{R}\right| \sim \omega$ and $($ F20 $)$, while for $\omega<\tilde{\omega}_{R}$ 
we use F21. In both instances it is simple to establish $|E(\omega)| \lesssim\left|\Delta_{I}\left(\omega_{R}\right)\right| / \omega_{R}$. To analyze the remaining case $\left|\omega-\tilde{\omega}_{R}\right| \ll \tilde{\omega}_{R}$ we consider the Taylor expansions of the functions $\Delta_{I}(\omega)$ and $I(\omega)$ around the frequency $\tilde{\omega}_{R}$. Equation (F17) implies that substituting into Eq. (F15) the linear parts of these expansions results in a relative error for $E(\omega)$ of order $\left|\omega-\tilde{\omega}_{R}\right| / \tilde{\omega}_{R} \ll 1$. Thus we may write

$$
\begin{aligned}
E(\omega) \approx & {\left[\frac{\partial \Delta_{I}}{\partial \omega}\left(\tilde{\omega}_{R}\right)-i \frac{\partial I}{\partial \omega}\left(\tilde{\omega}_{R}\right)-\frac{\Delta_{I}\left(\tilde{\omega}_{R}\right)}{\omega_{R}+\tilde{\omega}_{R}}\right] } \\
& \times \frac{\omega-\tilde{\omega}_{R}}{\omega-\tilde{\omega}_{R}+i \frac{\omega_{R}}{\omega_{R}+\omega} I\left(\tilde{\omega}_{R}\right)}
\end{aligned}
$$

which using again Eqs. (F17) and (F20) leads to the desired result for $E(\omega)$. This completes the derivation of Eq. 82 for the frequency range in which it is used in Subsection VB. It is worth noting that a straightforward derivation using Eqs. (31), (32), (F11), and (F15) leads to

$$
\left|\frac{\zeta(q)-\zeta_{*}(q)}{\zeta_{*}(q)}\right|=\left|\frac{\omega_{R}}{\omega_{R}+\omega} E(\omega)\right|
$$

with

$$
\zeta_{*}(q) \equiv \frac{\left\langle u_{R}^{\prime} \mid u_{q}\right\rangle}{2 \sqrt{\omega_{R} \omega_{q}}}\left[\left(\omega_{q}+\omega_{R}\right)\left(\omega_{q}-\tilde{\omega}_{R}\right)+i \omega_{R} I\left(\tilde{\omega}_{R}\right)\right]
$$

which can prove useful when analyzing the manipulation of a specific resonance by coupling to a pseudospin 20 .

Finally, to warrant our derivation of Eqs. (5) and (6) in Subsection $\mathrm{VB}$ we also need to establish that the function $I(\omega)$ remains well behaved at $\omega=\omega_{R}$ as $d / L \rightarrow 0$. This can be accomplished by using the exact expression (32), Eq. (26), and the behavior of the overlaps for small but finite $d / L$. To understand the latter it is essential to consider the qualitative behavior of corrections to the scattering modes $\phi_{k(q), \beta}(z)$. This can be done ${ }^{60}$ using: ( $i$ ) that each of the reflection amplitudes admits an expansion in powers of $k d$ and those corresponding to a junction relate to the finite transmission coefficient via energy conservation and $(i i)$ the exact reduction to a single junction performed in Sec. III. We note that for our present purposes the precise form of the coefficients of the expansion $(i)$, which will be studied elsewhere ${ }^{60}$, is not important. Propositions $(i),(i i)$, and Eq. 26 allow us to write the following Lorentzian approximation $L \equiv 1$ :

$$
\begin{gathered}
S(\omega) \approx A_{R}(d) \frac{\Gamma_{R}(d) / 2}{\left[\omega-\tilde{\omega}_{R}(d)\right]^{2}+\Gamma_{R}^{2}(d) / 4}, \\
\Delta_{S}(\omega) \approx A_{R}(d) \frac{\omega-\tilde{\omega}_{R}(d)}{\left[\omega-\tilde{\omega}_{R}(d)\right]^{2}+\Gamma_{R}^{2}(d) / 4}+B_{R}(d),
\end{gathered}
$$

where the corrections are higher order in $d$ for all frequencies in a small neighborhood of $\omega_{R}$, and to establish

$$
\lim _{d \rightarrow 0} A_{R}(d)=\frac{1}{2 \omega_{R}}, \quad \lim _{d \rightarrow 0} B_{R}(d)=0 .
$$

Inserting Eq. F25) into Eq. (32) we obtain

$$
\begin{gathered}
I(\omega) \approx \\
\frac{1}{\omega_{R} A_{R}(d)} \frac{\Gamma_{R}(d)}{2}\left(1+2 \frac{B_{R}(d)}{A_{R}(d)}\left[\omega-\tilde{\omega}_{R}(d)\right]\right. \\
\left.+\frac{B_{R}^{2}(d)}{A_{R}^{2}(d)}\left\{\left[\omega-\tilde{\omega}_{R}(d)\right]^{2}+\frac{\Gamma_{R}^{2}(d)}{4}\right\}\right)^{-1}
\end{gathered}
$$

which together with Eq. (F26) leads to $I(\omega) \approx \Gamma_{R}(d)$ for frequencies close to $\omega_{R}$ implying that (as expected on physical grounds) the resonator mode's environment is structureless at the characteristic resonant frequency $\omega_{R}$ (cf. Subsec. II B).

An analogous procedure allows to analyze the qualitative behavior at the other resonances $\omega=\omega_{n} \neq \omega_{R}$ where we find that $S(\omega)$ presents Fano profiles that result in corresponding features in the function $I(\omega)$ that have negligible relative spectral weight as $d / L \rightarrow 0$. Thus, though the latter do not affect the behavior at other frequencies, they invalidate Eqs. (F20), (F21) at these special frequencies precluding in their neighborhoods the use of Eq. 82. To conclude, we point out that from Eqs. (F25), (84), and 80 one can obtain an approximation for the function $S(\omega)$ [in terms of $\Gamma_{R}(d)$ ] adequate for all low frequencies other than $\omega_{n} \neq \omega_{R}$. The latter approximation together with the asymptotic behavior discussed at the end of Subsec. V B and the exact relation (32) allow for an independent heuristic justification of Eq. (F17) - i.e. without resort to the approximation 82 or the properties of the spectral densities expected on physical grounds.
* Electronic address: Ignacio.Wilson-Rae@ph.tum.de

1 H. G. Craighead, Science 290, 1532 (2000).

2 M. Roukes, Physics World 14, 25 (2001).

${ }^{3}$ K. L. Ekinci and M. L. Roukes, Review of Scientific Instruments 76, 061101 (2005).

${ }^{4}$ G. J. Milburn, K. Jacobs, and D. F. Walls, Phys. Rev. A 50, 5256 (1994).

${ }^{5}$ M. F. Bocko and R. Onofrio, Rev. Mod. Phys. 68, 755 (1996).

${ }^{6}$ K. C. Schwab and M. L. Roukes, Physics Today 58, 36
(2005).

7 J. A. Sidles, J. L. Garbini, K. J. Bruland, D. Rugar, O. Züger, S. Hoen, and C. S. Yannoni, Rev. Mod. Phys. 67, 249 (1995).

8 L. Thomas, F. Lionti, R. Ballou, D. Gatteschi, R. Sessoli, and B. Barbara, Nature 383, 145 (1996).

9 A. N. Cleland, Foundations of Nanomechanics (Springer, Berlin, 2003).

10 H. Gassmann, M.-S. Choi, H. Yi, and C. Bruder, Phys. Rev. B 69, 115419 (2004). 
11 D. F. Walls and G. J. Milburn, Quantum Optics (Springer, Berlin, 1994).

12 J. M. Raimond, M. Brune, and S. Haroche, Rev. Mod. Phys. 73, 565 (2001).

13 D. Leibfried, R. Blatt, C. Monroe, and D. Wineland, Rev. Mod. Phys. 75, 281 (2003).

14 S. Stenholm, Rev. Mod. Phys. 58, 699 (1986).

15 M. D. LaHaye, O. Buu, B. Camarota, and K. C. Schwab, Science 304, 74 (2004).

16 R. G. Knobel and A. N. Cleland, Nature (London) 424, 291 (2003).

17 E. M. Weig, R. H. Blick, T. Brandes, J. Kirschbaum, W. Wegscheider, M. Bichler, and J. P. Kotthaus, Phys. Rev. Lett. 92, 046804 (2004).

18 A. D. Armour, M. P. Blencowe, and K. C. Schwab, Phys. Rev. Lett. 88, 148301 (2002).

19 I. Martin, A. Shnirman, L. Tian, and P. Zoller, Phys. Rev. B 69, 125339 (2004).

${ }^{20}$ I. Wilson-Rae, P. Zoller, and A. Imamoglu, Phys. Rev. Lett. 92, 075507 (2004).

21 A. Hopkins, K. Jacobs, S. Habib, and K. Schwab, Phys. Rev. B 68, 235328 (2003); J. Eisert, M. B. Plenio, S. Bose, and J. Hartley, Phys. Rev. Lett. 93, 190402 (2004); A. Naik, O. Buu, M. D. LaHaye, A. D. Armour, A. A. Clerk, M. P. Blencowe, and K. C. Schwab, Nature 443, 193 (2006); L. F. Wei, Y. xi Liu, C. P. Sun, and F. Nori, Phys. Rev. Lett. 97, 237201 (2006); D. Vitali, S. Gigan, A. Ferreira, H. R. Bohm, P. Tombesi, A. Guerreiro, V. Vedral, A. Zeilinger, and M. Aspelmeyer, Phys. Rev. Lett. 98, 030405 (2007); D. Vitali, P. Tombesi, M. J. Woolley, A. C. Doherty, and G. J. Milburn, Phys. Rev. A 76, 042336 (2007). K. Jacobs, P. Lougovski, and M. Blencowe, Phys. Rev. Lett. 98, 147201 (2007); K. Jacobs, Phys. Rev. Lett. 99, 117203 (2007); J. D. Thompson, B. M. Zwickl, A. M. Jayich, F. Marquardt, S. M. Girvin, and J. G. E. Harris, Nature 452, 72 (2008); A. A. Clerk, F. Marquardt, and K. Jacobs (2008), arXiv:0802.1842.

22 B. Babić, J. Furer, S. Sahoo, S. Farhangfar, and C. Schönenberger, Nano Lett. 3, 1577 (2003).

23 V. Sazonova, Y. Yaish, H. Üstünel, D. Roundy, T. A. Arias, and P. L. McEuen, Nature (London) 431, 284 (2004).

24 L. Tian and P. Zoller, Phys. Rev. Lett. 93, 266403 (2004).

${ }^{25}$ M. Borgstrom, V. Zwiller, and E. M. A. Imamoglu, Nano Lett. 5, 1439 (2005).

26 P. Olivero, S. Rubanov, P. Reichart, B. C. Gibson, S. T. Huntington, J. Rabeau, A. D. Greentree, J. Salzman, D. Moore, D. N. Jamieson, et al., Adv. Mater. 17, 2427 (2005); A. D. Greentree, P. Olivero, M. Draganski, E. Trajkov, J. R. Rabeau, P. Reichart, B. C. Gibson, S. Rubanov, S. T. Huntington, D. N. Jamieson, et al., J. Phys.: Cond. Mat. 18, S825 (2006).

27 S. Mancini, D. Vitali, and P. Tombesi, Phys. Rev. Lett. 80, 688 (1998).

28 T. Kippenberg and K. Vahala, Optics Express 15, 17172 (2007).

29 C. H. Metzger and K. Karrai, Nature (London) 432, 1002 (2004); S. Gigan, H. R. Böhm, M. Paternostro, F. Blaser, G. Langer, J. B. Hertzberg, K. C. Schwab, D. Bäuerle, M. Aspelmeyer, and A. Zeilinger, Nature 444, 67 (2006); O. Arcizet, P.-F. Cohadon, T. Briant, M. Pinard, and A. Heidmann, Nature 444, 71 (2006); D. Kleckner and D. Bouwmeester, Nature 444, 75 (2006); A. Schliesser, P. DelHaye, N. Nooshi, K. Vahala, and T. Kippen- berg, Phys. Rev. Lett. 97, 243905 (2006); K. R. Brown, J. Britton, R. J. Epstein, J. Chiaverini, D. Leibfried, and D. J. Wineland, Phys. Rev. Lett. 99, 137205 (2007); A. Schliesser, R. Rivière, G. Anetsberger, O. Arcizet, and T. J. Kippenberg, Nature Physics 4, 415 (2008).

30 I. Wilson-Rae, N. Nooshi, W. Zwerger, and T. J. Kippenberg, Phys. Rev. Lett. 99, 093901 (2007); F. Marquardt, J. P. Chen, A. A. Clerk, and S. M. Girvin, Phys. Rev. Lett. 99, 093902 (2007); F. Xue, Y. D. Wang, Y. xi Liu, and F. Nori, Phys. Rev. B 76, 205302 (2007).

31 S. M. Carr, W. E. Lawrence, and M. N. Wybourne, Phys. Rev. B 64, 220101(R) (2001); P. Werner and W. Zwerger, Europhys. Lett. 65, 158 (2004); S. Savel'ev and F. Nori, Phys. Rev. B 70, 214415 (2004); S. Savel'ev, X. Hu, and F. Nori, New Journal of Physics 8, 105 (2006); S. Savel'ev, A. L. Rakhmanov, X. Hu, A. Kasumov, and F. Nori, Phys. Rev. B 75, 165417 (2007).

32 A. N. Cleland and M. L. Roukes, J. Appl. Phys. 92, 2758 (2002).

33 M. C. Cross and R. Lifshitz, Phys. Rev. B 64, 85324 (2001).

34 I. Wilson-Rae, Ph.D. thesis, University of California, Santa Barbara (2003).

35 D. M. Photiadis and J. A. Judge, Appl. Phys. Lett. 85, 482 (2004).

36 C.-M. Chang and M. R. Geller, Phys. Rev. B. 71, 125304 (2005).

37 A. O. Caldeira and A. J. Leggett, Ann. Phys. 149, 374 (1983).

38 U. Weiss, Quantum Dissipative Systems (World Scientific, 1999).

39 B. L. Hu, J. P. Paz, and Y. Zhang, Phys. Rev. D 45, 2843 (1992).

40 G. W. Ford, M. Kac, and P. Mazur, J. Math. Phys. 6, 504 (1965).

41 L. G. C. Rego and G. Kirczenow, Phys. Rev. Lett. 81, 232 (1998).

42 K. C. Schwab, E. A. Henriksen, J. M. Worlock, and M. L. Roukes, Nature (London) 404, 974 (2000).

${ }^{43}$ R. Lifshitz and M. L. Roukes, Phys. Rev. B 61, 5600 (2000).

44 H. Jiang, M.-F. Yu, B. Liu, and Y. Huang, Phys. Rev. Lett. 93, 185501 (2004).

45 L. D. Landau and E. M. Lifshitz, Theory of Elasticity (Butterworth-Heinemann, Oxford, 1986).

46 K. F. Graff, Wave Motion in Elastic Solids (Dover, New York, 1991).

47 D. E. Segall, S. Ismail-Beigi, and T. A. Arias, Phys. Rev. B 65, 214109 (2002).

48 P. Ullersma, Physica 32, 27 (1966).

49 P. Ullersma, Physica 32, 56 (1966).

50 C. Viviescas and G. Hackenbroich, Phys. Rev. A 67, 013805 (2003), and references therein.

51 G. Anetsberger, R. Rivière, A. Schliesser, O. Arcizet, and T. J. Kippenberg (2008), arXiv:0802.4384.

52 B. I. Yakobson, C. J. Brabec, and J. Bernholc, Phys. Rev. Lett. 76, 2511 (1996).

53 D. H. Robertson, D. W. Brenner, and J. W. Mintmire, Phys. Rev. B 45, 12592 (1992).

54 P. Dutta and P. M. Horn, Rev. Mod. Phys. 53, 497 (1981).

55 E. Paladino, L. Faoro, G. Falci, and R. Fazio, Phys. Rev. Lett. 88, 228304 (2002).

56 R. S. Phillips and P. D. Lax, Scattering theory (Academic Press, 1989). 
57 V. Hakim and V. Ambegaokar, Phys. Rev. A 32, 423 (1985).

58 A. Messiah, Quantum Mechanics (Dover, New York, 1961).

${ }^{59}$ K. Wang, A.-C. Wong, and C. T.-C. Nguyen, J. Microelectromechanical Syst. 9, 347 (2000).

${ }^{60}$ I. Wilson-Rae (unpublished).

61 E. W. Weisstein, URL http://mathworld.wolfram.com/ TitchmarshTheorem.html.

${ }^{62}$ More precisely, at the origin taken as the center of mass of the beam's cross section at the junction. We note that the anomalous frequency scaling of the $\tilde{\rho}_{\beta}(\omega)$ [cf. Eq. (58)] when viewed as the DOS of a $1 \mathrm{D}$ fictitious environment expresses just the non-adiabatic nature of the junction.

63 This yields $k_{n, c / t}=\pi(n+\delta / 2) / L$ and $k_{n, v / h} \approx \pi(n+\delta-$ $1 / 2) / L$ where the relative error is of order $e^{-k_{n, v / h} L}$.

${ }^{64}$ More precisely, they should yield the correct order of magnitude provided the corresponding resonance lies below the lowest infrared cutoff associated with the phonon waveguide afforded by the undercut - here we assume $d_{U} \sim L \gg \mathrm{t}$.

65 The criterion underpinning the derivation is that the support-induced frequency shift (see below) be smaller than the "free spectral range" associated with the bare resonance.

${ }^{66}$ If there are support dimensions $d_{s} \lesssim d$ our derivations for the lowest order in $d / L$ remain valid provided the limit $d \rightarrow$ 0 yields a support satisfying assumption $(i)$. Implicit in the latter is the idealization that for a bridge the two supports are effectively disconnected for phonons with wavelengths $\lesssim 2 \pi / L$.

67 Note that the new set of modes $\left\{\bar{u}_{R}^{\prime}(\bar{r}), \bar{u}_{q}^{\prime}(\bar{r})\right\}$ is chosen to be also orthonormal in the metric defined by Eq. (19) - which accounts for the discrete mode's elastic energy density being proportional to $\rho_{s}$ rather than $\rho_{b}$.

68 More precisely, its analog with $\omega_{R}^{2} \rightarrow \omega_{R}\left[\omega_{R}-\Delta_{I}(0)\right]$ [cf. Eqs. 21], 25 and Eq. 833]. This correction corresponds to the environment-induced renormalization of the resonator's frequency that does not cancel out given the lack of counterterm in Hamiltonian 20.

${ }^{69}$ We note that while $\bar{u}_{R}^{\prime}(\bar{r}) e^{-i \omega_{R} t}$ is only a solution of the elastic wave equation inside the resonator, the spatial average of the elastic energy density should be taken over the whole structure when considering Hamiltonian $H$. However, the boundary term that arises due to the discontinuity at the junction vanishes due to the choice of homogeneous boundary conditions. The reader is referred to Ref. 50, where a related treatment is given of the electromagnetic analog of our problem.

70 Note that we consider the limit $d / L \rightarrow 0$ with $k(q) L$ held constant.

${ }^{71}$ For the last two properties, in the case of the TRE solutions, we have also used that $|\bar{r}| \mathbf{G}\left(\bar{r}-\bar{r}^{\prime}, \omega\right)$ is well behaved as $\omega \rightarrow 0$.

72 These may be chosen to have well defined reflection symmetries as for the free elastic half space.

73 This is just the analog for the decoupled support of Eq. 18 and is obtained from it via the ansatz $\bar{u} \rightarrow \bar{u}^{(0)}, b \rightarrow b^{(\sigma)}$.

74 The limits $S \rightarrow 0$ of the angles are taken keeping the shape of the cross section invariant.

75 The 2D surface states (SAW) have a penetration depth that scales as $1 / q_{t}$ so that their contribution to the DOS has the same scaling with $\omega$ as the bulk contribution.

${ }^{76}$ We note that as the cross section $S$ lies at $z=0$ one has $M_{i_{1}, i_{2} \ldots i_{n+1}}^{(n)}=0$ for $i_{m}=3$ with $m \neq 1$.

77 Though the universal properties discussed in App. B imply that for the end corrections the total force [zeroth order moment $M^{(0)}$ ] and total torque [antisymmetric part of $M^{(1)}$ ] scale at most as the inertia, the symmetric part of $M^{(1)}$ and the $M^{(2)}$ can be zeroth order in $k$.

78 There is naturally a purely real singular part that corresponds to the quasistatic near field and over an oscillation period does not perform net work.

79 Note that the $\phi_{n, \beta}(z)$ are homogeneous solutions of the linear systems defined by the RHS of Eqs. 34, (36) in the limit $d / L \rightarrow 0$.

80 This disparity is just a trivial consequence of the dispersion relations (which imply $\omega_{0, t} / \omega_{0, v / h} \sim L / d$ ) and the strong dependence of the corresponding $Q$-values with the aspect ratio - for similar dimensions the $Q$-values of the fundamental resonances of these branches are comparable.

81 For asymmetric cross sections the $\beta$ index is conventional.

82 Any surface or edge states resulting from the free boundaries have a penetration depth of order $1 / q_{t}$.

${ }^{83}$ For a SWNT if we use the shell model the appropriate ansatz reads instead $S \rightarrow 2 \pi R, I_{z} \rightarrow 2 \pi R\left\langle r^{2}\right\rangle_{S}$ - naturally, the results for the functions $\tilde{\rho}_{\beta}(\omega)$ are independent of the "microscopic model" used [cf. Eqs. 447, (50), (56)].

84 In instances where there are several disparate characteristic wavelengths - as is the case for the radial breathing mode of a microtoroid that hybridizes appreciably with the flexural modes of the corresponding membrane $e^{51}$ $k_{R}$ should be taken as the largest of the corresponding wavevectors.

${ }^{85}$ An example of particular relevance in the context of optomechanics is the radial breathing mode of a microtoroid $28 \mid 51$

${ }^{86}$ In the case of microtoroids with membrane thickness ${ }^{28 \mid 51} h$ much smaller than the smallest pedestal radius one should instead define the resonator volume as the undercut region, so that $S$ corresponds to the vertical rim at the smallest radius of the undercut. Thus, this scenario falls into the singular case $(i)$ as defined below Eq. 10 and $\bar{u}_{R}^{\prime}$ should instead be specified by clamped boundary conditions at $S$.

87 It is straightforward to extend the derivation to resonances with other reflection symmetries $\hat{R}_{x}, \hat{R}_{y}$ - as for the symmetric beam geometry each resonance only couples to the TRE branch with the same symmetries.

88 To understand the normalization and the support's onedimensional DOS (needed below to integrate over $q$ ) it is instructive to consider the infinite length limit of the elastic object generated by reflecting the pedestal with respect to $S$ and identifying the resulting endpoints - with an adequate smoothening of the possible discontinuities in $S^{\prime}(z)$.

89 Note that $\bar{u}_{R}^{\prime}$ is normalized using the non-trivial metric defined by Eq. (19). 